\title{
Numerical Simulation of Matrix Reinforced Composite Materials Subjected to Compression Loads
}

\author{
Xavier Martinez $\cdot$ Sergio Oller
}

Received: 15 June 2009 / Accepted: 15 June 2009 / Published online: 14 August 2009

(C) CIMNE, Barcelona, Spain 2009

\begin{abstract}
This paper reviews the most common formulations to obtain the compression strength of long fiber composites due to fiber buckling. This failure mode was first studied by Rosen (Fibre Composite Materials, pp. 37-45, 1965) who defined two different fiber buckling modes, extensional and transverse. Further studies improved the first model proposed by Rosen by defining with more accuracy the mechanics of the problem. Although each formulation use a different approach to solve the problem, all of them agree in the dependence of fiber buckling on three main parameters: matrix shear strength, fiber initial misalignments and volumetric participation of the fibers in the composite.

Once having described the different approaches used, and the parameters on which they depend, this paper describes a new formulation capable of obtaining the compression strength of composites taking into account the fiber buckling
\end{abstract}

This work has been supported by the Spanish Ministry of Science and Innovation throughout the projects "RECOMP", Ref.

BIA2005-06952, "DECOMAR", Ref. MAT2003-08700-C03-02 and

"DELCOM", Ref. MAT2008-02232/MAT; by the Spanish Ministry of Public Works project "Retrofitting and Reinforcement of Reinforced Concrete structures with Composite Materials"; by "AIRBUS" (Spain), Contract No. PBSO-13-06 "FEMCOM", by "ACCIONA Infraestructuras" (Spain) throughout the projects "CETIC", "Sphera" and "Prometeo"; by Agencia Española de Cooperación Internacional para el Desarrollo (AECID), Ref. A/012257/07; and by International Center for Numerical Methods in Engineering (CIMNE), Spain. All support is gratefully acknowledged.

X. Martinez ( $\varangle)$

CIMNE, Gran Capità, s/n, 08034 Barcelona, Spain

e-mail: xaviermg@cimne.upc.edu

S. Oller

Universitat Politecnica de Catalunya (UPC), Barcelona, Spain

e-mail: sergio.oller@upc.edu phenomenon. This formulation uses the serial/parallel mixing theory developed by Rastellini et al. (Comput. Struct. 86(9):879-896, 2008) to simulate the composite, and takes advantage of knowing the mechanical performance of the composite constituents to simulate the fiber buckling phenomenon. This is done with an homogenization procedure. It consists in introducing the interaction between fibers and matrix into their respective constitutive equations. The interaction between fiber and matrix takes into account fiber initial misalignments, its volumetric participation and the mechanical properties of both constituents.

The new formulation proposed is implemented in a finite element code, taking into account that fibers can have different misalignment levels, and that the composite behaves differently if it is under tensile or compression forces. The mechanical performance of the formulation proposed is studied with several finite element simulations of compressed composites. Finally, the correctness of the formulation is proved by comparing the numerical results with the experimental tests provided by Barbero and Tomblin (Int. J. Solids Struct. 33(29):4379-4393, 1996), Tomblin et al. (Int. J. Solids Struct. 34(13):1667-1679, 1997).

\section{Introduction}

The failure of long fiber composites subjected to compressive forces can be due to two main reasons, fiber buckling and/or composite delamination [5]. Regarding the fiber buckling phenomenon, first studies correspond to Rosen [1], who defined two different buckling modes and the compression stress at which buckling occurs. Rosen expression depends on matrix shear strength and on the volumetric participation of fibers in the composite. This first formulation led to new studies that tried to predict with more accuracy the composite critical compression stress due to fiber buck- 


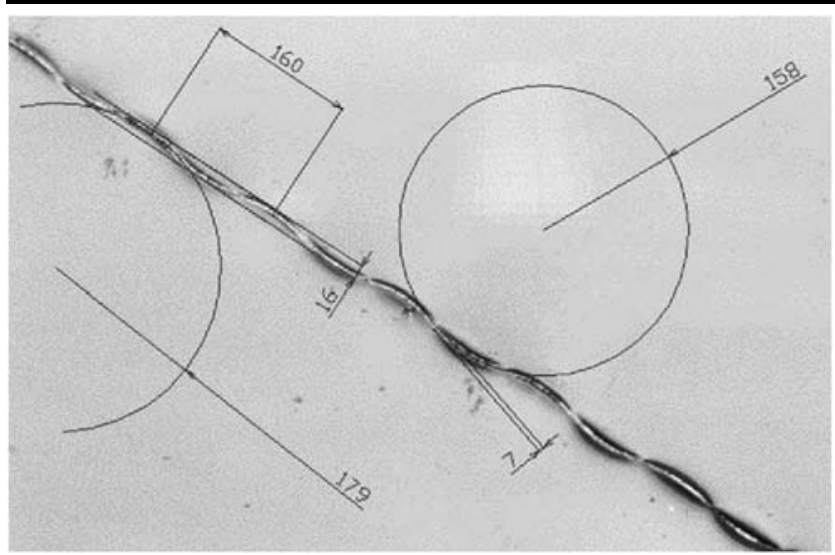

Fig. 2. Example of microbuckling measurements (in micrometers) done on a T300 carbon fibre inside of an LY556 epoxy matrix.

Fig. 1 Initial fiber misalignments [15]

ling. Of those, its worth to mention the works of Barbero and Tomblin [3, 4, 6], Balacó de Morais [7-9] and Drapier et al. $[10,11]$. The models proposed by these authors are based on energetic equilibriums between the composite components, these equilibriums lead to a final expression of the maximum compression stress that can be applied to the composite. It is also interesting the scope used bs

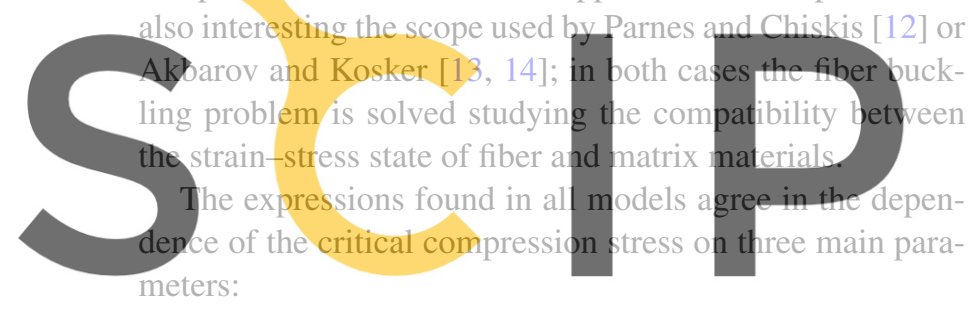

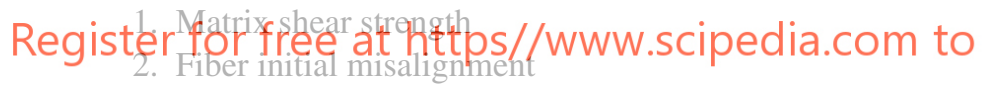

3. Volumetric participation of fiber and matrix in the composite

So, the composite compression capacity of these new formulations depends on the same parameters pointed out by Rosen and on a new one: fiber initial misalignments. Therefore, any formulation regarding fiber micro-buckling, must take into account fiber initial misalignments if it wants to predict accurately composite compression strength.

According to Jochum and Grandidier [15], fiber misalignments are produced in the composite manufacturing process, during the matrix curing. These misalignments are regular in frequency and amplitude along the whole fiber length and can be represented by a sinusoidal shape. Figure 1 shows a micro-photograph of the misalignments found in a carbon fiber embedded in an epoxy matrix.

There are two main problems in all known formulations developed to obtain the compression strength of the composite. The first one is that most of them only provide an expression to obtain the maximum stress that can be applied to the composite before fiber buckling occurs. However, in a structural problem, a local failure does not imply the total failure of the structure, being necessary to know the postcritical behavior of the material to continue the simulation, if the global performance of the structure wants to be obtained.

The second problem appears because the composite is treated as a single material with average properties from its constituents. A formulation focused only in the composite, forces to pre-define the constitutive performance of the composite constituents in order to obtain the expressions that will provide the critical buckling strength of the material. In example, Drapier et al. [10] consider that the fibers are an elastic material and the matrix is modeled with a J2 law. Thus, the application range of the formulation developed is reduced to those composites which constituents have an specific constitutive performance, being necessary to reformulate the whole problem if a different constitutive law is required.

These two problems: impossibility to obtain the postcritical strength of the composite and pre-defined constitutive behavior of the composite components, can be solved simulating the composite material with the serial/parallel mixing theory developed by Rastellini [2]. This theory can be understood as a constitutive equations manager, that ob-

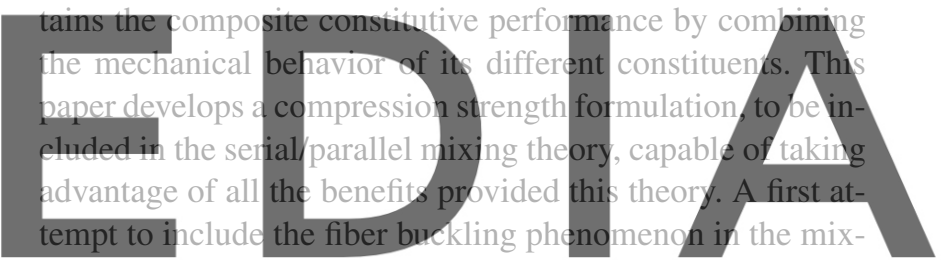
ing theory has been already made by Puig et al. [16], with

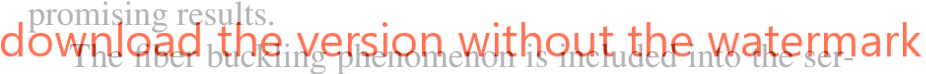
ial/parallel mixing theory by modifying the constitutive performance of the composite constituent materials (fiber and matrix) based on the micro-structural interaction that exist between them. This approach transforms a cinematical problem, structural instability due to fiber buckling, into an equivalent constitutive problem; reducing significantly the computational cost of the simulation. The modification of the constitutive performance of both components is made using an homogenization method.

Homogenization methods deal with the global problem of composite material in a two scale context $[17,18]$. The macroscopic scale works with the composite material to obtain the global structural response; composites are treated as homogeneous materials in this scale. The microscopic scale corresponds to an elemental characteristic volume in which the microscopic fields inside the composite are obtained, this scale works with the component materials of the composite, studying the behavior of a Representative Volume Element (RVE). Homogenization theory assumes a periodical repetition of the RVE in the composite material to relate these two scales. 


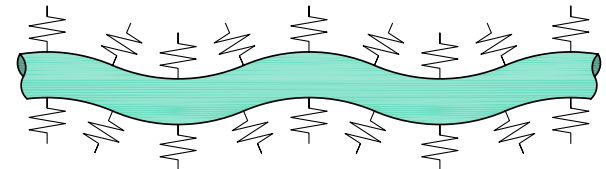

Fig. 2 Problem of a curved bar under unilateral elastic restriction $(\mathrm{Cu}-$ BER problem)

The study of the fiber buckling problem using an homogenization method would consist on obtaining the composite performance by solving an RVE with a finite element model of the fiber-matrix system, using a large displacement theory to obtain the moment in which fiber buckles, and taking into account the initial fiber misalignments. The results obtained from this model have to be extrapolated to the composite in order to solve the structural problem.

Homogenization methods are unaffordable to be used nowadays due to their high computational cost: obtaining the response of a real structure with this methods implies solving a complex finite element model for each point in which the structure is discretized. Therefore, most of the research performed in the homogenization field is focused on the study of the structural performance of RVE's [19] or on how the information can be transferred from the micro-scale

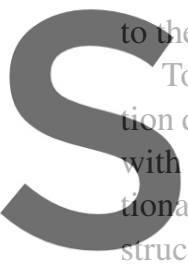
e macro-scale $[20,21]$.
developed, the finite element
an homogenization method is
al case using the existing ana
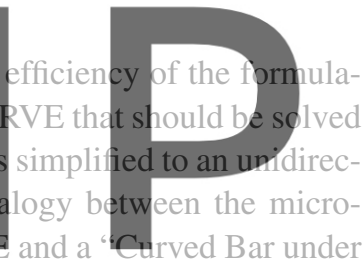
unilateral Elastic Restrain" (CuBER problem, Fig. 2). It will

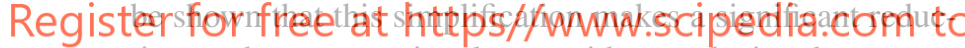
tion on the computational cost, without reducing the amount of information obtained, neither the accuracy of it.

The homogenization method used consist in transforming the structural solution provided by the CuBER problem, that characterizes the interaction between fiber and matrix, into some parameters to be included in the constitutive equations of both composite components: fiber and matrix. Thus, what is homogenized is not the composite but its constituents. Once having a modified constitutive behavior of the composite constituents, the serial/parallel mixing theory can be used to obtain a composite performance, that will take into account the micro-structural interaction of fiber and matrix due to fiber initial misalignments. It will be shown that the new constitutive performance of both materials, together with the mixing theory formulation, is capable to simulate the fiber buckling phenomenon, obtaining the critical compression stress of the composite and its post-critical behavior. Also, the formulation developed establishes a direct relation between the compression strength of the composite and the three main parameters defined in literature to obtain this strength: fiber initial misalignments, matrix shear strength and volumetric participation of fiber and matrix in the composite.

This paper is structured as follows. The following section describes the formulation used to simulate composite materials: the serial/parallel mixing theory. Section 3 contains a brief state of the art of the different theories and formulations existing nowadays to obtain the compression strength of composite materials. Afterwards, Sect. 4 describes the formulation required to solve the CuBER problem, as well as the methodology used to transform the results obtained from the CuBER problem into two homogenized constitutive laws, one for the fiber and the other for the matrix. These modified constitutive laws are used by the mixing theory. The validation of the new homogenized constitutive equations is shown in Sect. 5, where the results obtained with the new formulation are compared with the results that are obtained from a micro-model of the RVE cell considered. Once having proved the correct performance of the methodology proposed, Sect. 6 defines the algorithm used to implement in a finite element code the formulation developed. The validation of the algorithm proposed is described in Sect. 7. This validation compares the numerical results with experimental data existing in literature. Finally, Sect. 8 includes the conclusions obtained from the formulation described, and

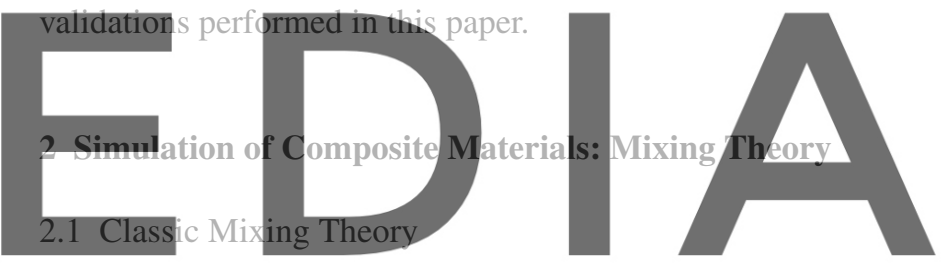

The classical rule of mixtures, or mixing theory, deals with

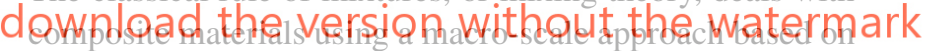

the mechanics of continuous mediums. The composite mechanical behavior is obtained from the constitutive performance of its component materials; being possible to include the material non-linearities of the constituents into the composite mechanical performance. This approach represents a substantial improvement compared to other existing formulations developed to deal with composite materials, as these only provide an accurate prediction of the composite mechanical performance when its constituents are in their elastic range. Another advantage of the mixing theory is that it can use all existing numerical procedures and formulations already developed to simulate continuum materials to obtain the performance of the composite constituents.

\subsubsection{Direct Mixing Theory}

The first formulation of the rule of mixtures corresponds to Trusdell and Toupin [22]. This first work became the base of future studies developed by Ortiz and Popv [23, 24] and by Green and Naghdi [25]. The classical rule of mixtures defines the way in which all substances composing a material 
interact to provide the material performance. This interaction is defined with the following hypothesis:

i. Each infinitesimal volume of the composite contains a finite number of material components.

ii. Each component contribution to the global behavior of the composite is proportional to its volumetric participation.

iii. All components suffer the same strains (closing equation).

iv. The volume of each component is significantly smaller than the composite volume.

The closing equation, defined in condition (iii), defines an iso-strain field for all composite components. This can be written as:

${ }^{c} \varepsilon={ }^{1} \varepsilon=\ldots={ }^{s} \varepsilon=\ldots={ }^{n} \varepsilon$

Being ${ }^{c} \varepsilon$ the composite strain, ${ }^{s} \varepsilon$ the strain in the composite component $s$ and $n$ the total number of components in the composite.

The stress field of the composite is obtained using the second hypothesis of the mixing theory:
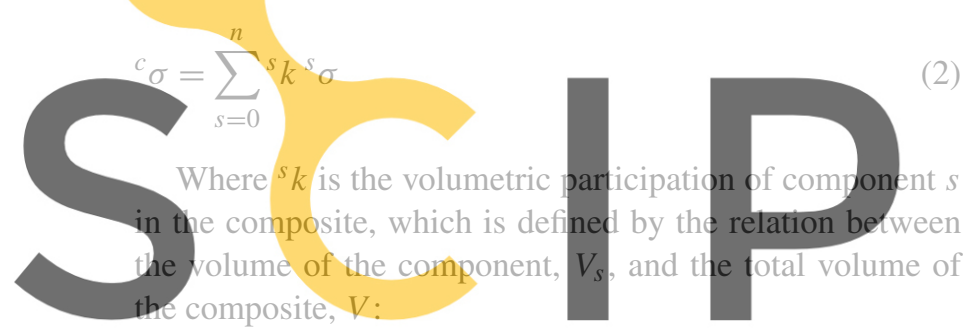

$(2)$

\section{Register for freeen attrittep//www.scipedia.com(to}

The relation between the strain and the stress fieids is defined with the material constitutive tensor, C. Thus, the stress in the composite can be obtained from its strain field using (1) and (2):

$$
\begin{aligned}
{ }^{c} \sigma & =\sum_{s=0}^{n}{ }^{s} k^{s} \sigma=\sum_{s=1}^{n}{ }^{s} k\left({ }^{s} \mathbf{C}:{ }^{s} \varepsilon\right) \\
& =\sum_{s=1}^{n}{ }^{s} k\left({ }^{s} \mathbf{C}:{ }^{c} \varepsilon\right)=\left(\sum_{s=1}^{n}{ }^{s} k^{s} \mathbf{C}\right):{ }^{c} \varepsilon
\end{aligned}
$$

This last equation provides the expression of the composite constitutive tensor, which is defined as:

${ }^{c} \mathbf{C}=\sum_{s=1}^{n}{ }^{s} k{ }^{s} \mathbf{C}$

The iso-strain hypothesis defined in the mixing theory implies a parallel distribution of the components in the composite. Depending on the direction in which the loads are applied to it, there are some situations in which the composite performance is better represented by an iso-stress relation between its components. This case is solved with the inverse mixing theory.

\subsubsection{Inverse Mixing Theory}

The inverse mixing theory replaces the iso-strain assumption by an iso-stress assumption, therefore:

${ }^{c} \sigma={ }^{1} \sigma={ }^{2} \sigma=\ldots={ }^{n} \sigma$

And, the strains in the composite are obtained according to the volumetric participation of each component material:

${ }^{c} \varepsilon=\sum_{s=0}^{n} s^{s}{ }^{s}$

Making use of the relation between strains and stresses, provided by the stiffness tensor, it can be obtained the relation between the stress and the strain field in the composite as:

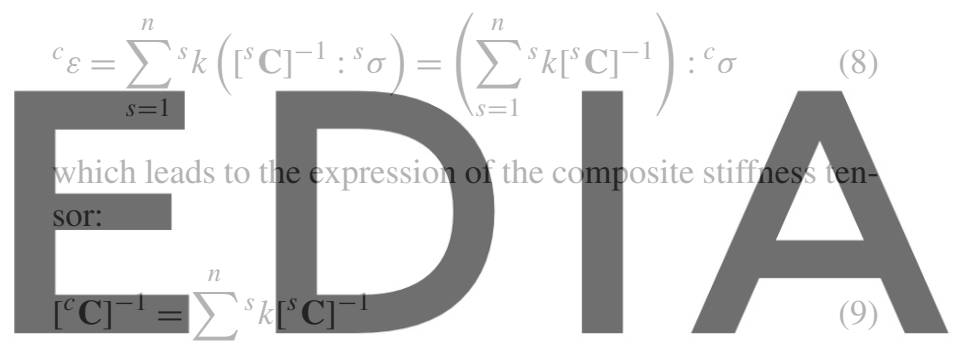

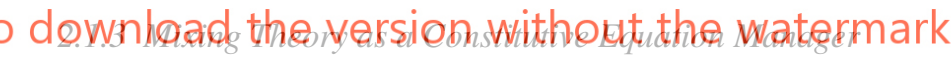

Oller et al. [26] and Car [27], coupled the mixing theory hypothesis with a thermodynamical description of the composite components. This provides a formulation for composite materials that obtains the relation between components, even when they have reached their elastic limit.

This new approach defines the Helmholtz free energy of the composite, ${ }^{c} \Psi$, as the sum of the free energies of each composite component, proportional to their volumetric participation. This is:

$$
\begin{aligned}
{ }^{c} \Psi\left({ }^{c} \varepsilon^{e}, \theta,{ }^{c} \alpha^{m}\right) & ={ }^{c} \Psi(\varepsilon, \theta, \underbrace{{ }^{c} \varepsilon^{p},{ }^{c} \alpha^{m}}_{{ }^{c} p_{s}}) \\
& =\sum_{s=1}^{n}{ }^{s} k^{s} \Psi\left(\varepsilon, \theta,{ }^{s} p_{s}\right)
\end{aligned}
$$

Being $\varepsilon, \varepsilon^{e}$ and $\varepsilon^{p}$ the total, elastic and plastic strains (the total strain is the same for the composite and its constituents, according to the mixing theory hypothesis), $\theta$ the temperature and $\alpha^{m}, p_{s}$ sets of internal variables. 
Fig. 3 Implementation of the mixing theory in a finite element code [27]
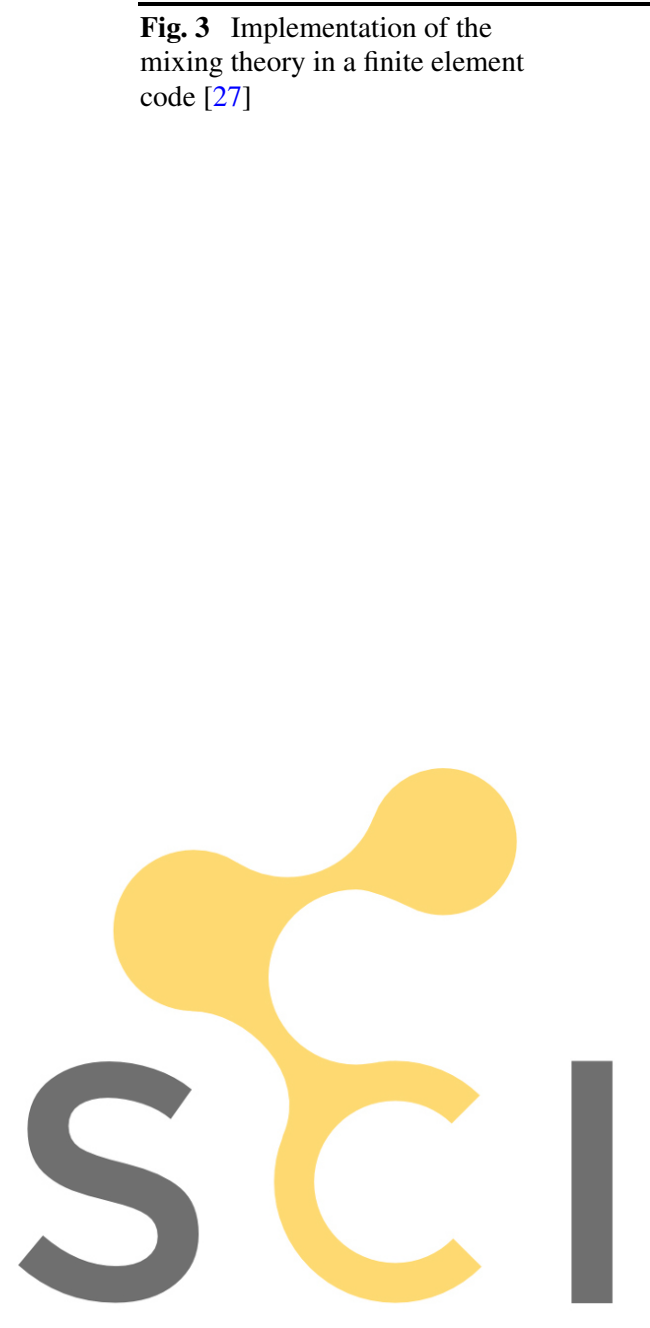

Once having defined the free energy of the composite in function of the free energies of its constituents, the procedure described in [28-30] to obtain the stress of single-phase materials from the free energy can be applied to both sides of (10), obtaining:

${ }^{c} \sigma=\sum_{s=1}^{n}{ }^{s} k^{s} \sigma$

It is worthy to remark that although (2) and (11) look exactly the same, their meaning is not. Equation (11) is obtained following a thermodynamic procedure, which means that the stress values can be obtained using any constitutive law based on thermodynamic principles, such as plasticity or damage. Therefore, the mixing theory has been transformed in a constitutive equations manager: it obtains the performance of the composite coupling the constitutive performance of its constituents.
The implementation of the mixing theory, as a constitutive equation manager, into a finite element code has been done by Oller et al. [26] and afterwards improved by Car [27] in his $\mathrm{PhD}$ dissertation, including in it the large deformation theory. The implementation algorithm developed by Car is shown in Fig. 3.

The validity of the new procedure developed to obtain the mechanical performance of composite materials, even when the composite constituents have reached its elastic yield stress, is proved in the numerical simulations described in $[27,31,32]$.

This new procedure has the problem already pointed out when describing the classical mixing theory developed by Trusdell and Toupin [22], the closing equation assumes an iso-strain performance of the composite and its constituents, which forces a parallel behavior of the composite components (Fig. 4a). In most cases this assumption is valid because composites are designed to work in the fiber direction. 
Fig. 4 Serial-Parallel distribution of the components in a composite material

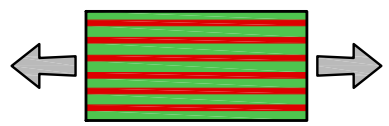

(a) Parallel behavior

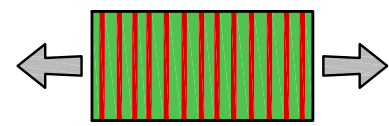

(b) Serial behavior

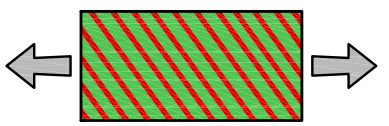

(c) Mixed mode behavior

However, depending on the direction of the loads applied other behaviors can be developed: a serial behavior (Fig. 4b) or, most commonly, a mixed mode behavior (Fig. 4c).

Oller et al. [33, 34] made a first attempt to develop a formulation of the mixing theory capable to take into account all possible behaviors of the composite: parallel, serial and mixed. This formulation defines the composite strains as a proportional sum of the serial and parallel strains found in it:

${ }^{c} \varepsilon_{i j}=(1-\aleph)^{c} \varepsilon_{i j}^{p a r}+\aleph^{c} \varepsilon_{i j}^{s e r}$

Where $\varepsilon_{i j}^{p a r}$ and $\varepsilon_{i j}^{s e r}$ stand for the parallel and serial strains, respectively, and $\aleph$ is the proportionality parameter, that depends on the fiber orientation. This parameter has to be obtained from experimental data. The serial and parallel strains of the composite are obtained as a function of the strains of the constituent materials, ${ }^{s} \varepsilon_{i j}$, as follows:

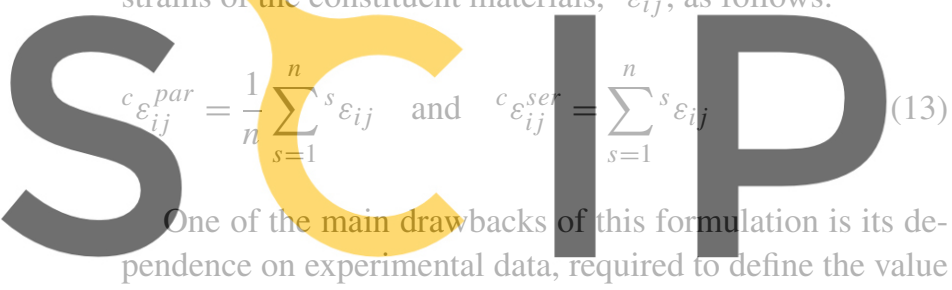
of $\aleph$. Recently, Rastellini et al. [35, 36] have developed a

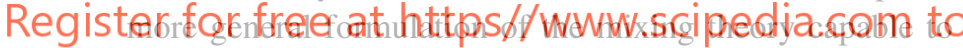
take into account the serial and parallel performance of the composite automatically, without the need of experimental or calibration parameters. This formulation is explained in the following section.

\subsection{Serial/Parallel Mixing Theory}

The serial/parallel rule of mixtures is an improvement of the classical mixing theory, in which the iso-strain hypothesis is replaced by an iso-strain condition in the fiber direction and an iso-stress condition in the transversal directions [2].

\subsubsection{Definition of the Serial and Parallel Components of the Strain and Stress Tensors}

The serial/parallel (SP) model considers that the constituent materials of the composite act in parallel in a certain direction and in serial in the remaining directions. Consequently, it is necessary to define and separate the serial and parallel components of the strain and stress tensors.
Defining $e_{1}$ as the director vector that determines the parallel behavior (fiber direction), the parallel projector tensor $N_{P}$ can be defined as follows:

$N_{P}=e_{1} \otimes e_{1}$

From $N_{P}$, the 4 th-order parallel projector tensor, $P_{P}$, is defined as:

$P_{P}=N_{P} \otimes N_{P}$

The serial projector tensor $P_{S}$ is evaluated as its complement:

$P_{S}=I-N_{P}$

Both tensors can be used to find the parallel part of the strain tensor $\varepsilon_{P}$ and its serial part $\varepsilon_{S}$ :

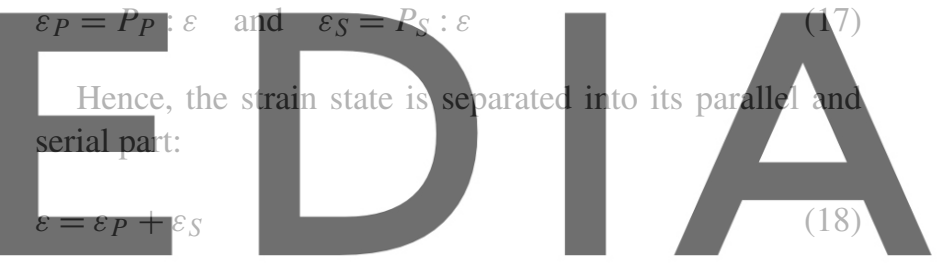

The stress state can be separated analogously, finding its dowathloads the prersiong the ithoutterthes ow ate and a rk $P_{S}$ as:

$\sigma=\sigma_{P}+\sigma_{S}$

where,

$\sigma_{P}=P_{P}: \sigma \quad$ and $\quad \sigma_{S}=P_{S}: \sigma$

\subsubsection{Numerical Modeling Hypotheses}

The numerical model developed to obtain the strain-stress state in the composite is based on the following hypotheses:

i. The constituent materials of the composite are subjected to the same strain in the parallel direction. The parallel direction corresponds to fiber orientation.

ii. The constituent materials are subjected to the same stress in the serial direction.

iii. The response of the composite material is directly related to the volume fractions of its constituent materials.

iv. The phases in the composite are considered to be homogeneously distributed. 
v. The constituent materials are considered to be perfectly bonded.

Although these hypotheses, as well as the serial/parallel mixing theory, can be applied to composites with any number of components, the developed formulation is restricted to only two of them. Therefore, for the sake of consistency, only two composite constituents are included in the theory description: fiber and matrix.

\subsubsection{Equilibrium and Compatibility Equations}

The equations that define the stress equilibrium and establish the strain compatibility between the individual components follow the hypotheses previously described. These are:

Parallel behavior:

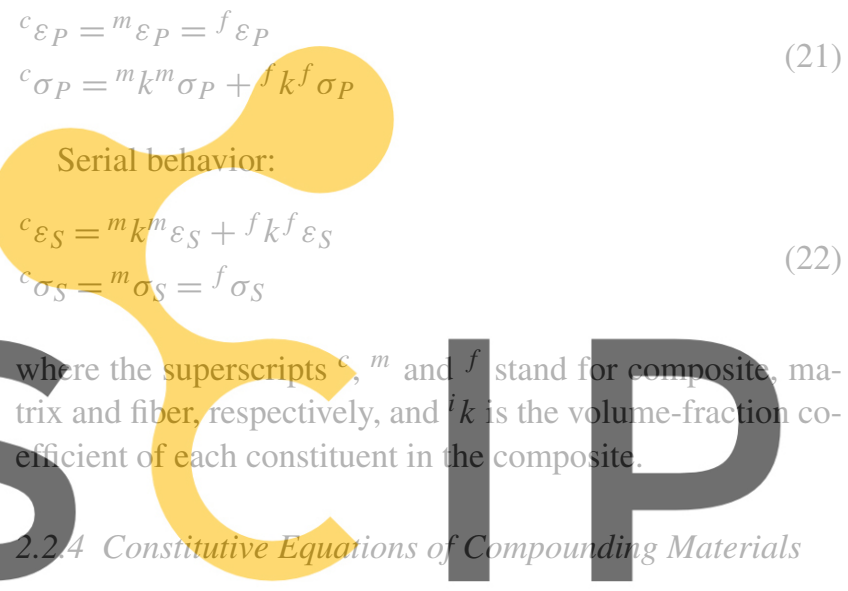

When applying the serial/parallel mixing theory, it is possi-

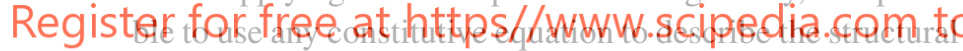
performance of each composite compounding material. The constitutive equations used can be different for each component (i.e. an elastic law to describe the fiber behavior and a damage formulation to describe the matrix behavior). Considering that additive plasticity is used to formulate the constitutive equations of the materials, the stresses in the matrix and the fiber are obtained as:

${ }^{m} \sigma={ }^{m} \mathbf{C}:\left({ }^{m} \varepsilon-{ }^{m} \varepsilon^{p}\right)$

$f_{\sigma}={ }^{f} \mathbf{C}:\left(f_{\varepsilon}-f_{\varepsilon}{ }^{p}\right)$

where ${ }^{m} \varepsilon^{p}$ and ${ }^{f}{ }_{\varepsilon}^{p}$ are the matrix and fiber plastic-strain tensors, respectively, and ${ }^{m} \mathbf{C}$ and ${ }^{f} \mathbf{C}$ are the matrix and fiber constitutive tensors.

These equations can be rewritten to consider the serial and parallel separation of the strain and stress tensors ((18) and (19))

$\left[\begin{array}{c}{ }^{i} \sigma_{P} \\ { }^{i} \sigma_{S}\end{array}\right]=\left[\begin{array}{cc}{ }^{i} \mathbf{C}_{P P}{ }^{i} \mathbf{C}_{P S} \\ { }^{i} \mathbf{C}_{S P}{ }^{i} \mathbf{C}_{S S}\end{array}\right]:\left[\begin{array}{c}{ }^{i} \varepsilon_{P}-{ }^{i} \varepsilon_{P}^{p} \\ { }^{i} \varepsilon_{S}-{ }^{i} \varepsilon_{S}^{p}\end{array}\right]$ where,

$\left\{\begin{array}{l}{ }^{i} \mathbf{C}_{P P}=P_{P}:{ }^{i} \mathbf{C}: P_{P} \\ { }^{i} \mathbf{C}_{P S}=P_{P}:{ }^{i} \mathbf{C}: P_{S} \\ { }^{i} \mathbf{C}_{S P}=P_{S}:{ }^{i} \mathbf{C}: P_{P} \\ { }^{i} \mathbf{C}_{S S}=P_{S}:{ }^{i} \mathbf{C}: P_{S}\end{array} \quad\right.$ with $i=m, f$

\subsubsection{Serial/Parallel Rule of Mixtures Algorithm}

The strain state of the composite material, ${ }^{c} \varepsilon$, at time $t+\Delta t$ is the known variable entered into the algorithm. Using this variable, the serial/parallel rule of mixtures algorithm has to determine the strain and stress states of each component that fulfills the equilibrium, the compatibility and the constitutive equations and the evolution of the internal variables.

The first step of the algorithm is to separate the strain tensor into its parallel and its serial components, in order to compute the strain state in the matrix and the fiber. According to (21), the parallel strain component is the same for both materials and for the composite. However, to determine the serial strains, it is necessary to predict the expected strains in one of the composite compounding materials. If this prediction is made for the matrix, the algorithm computes its serial strain increment as [2]:

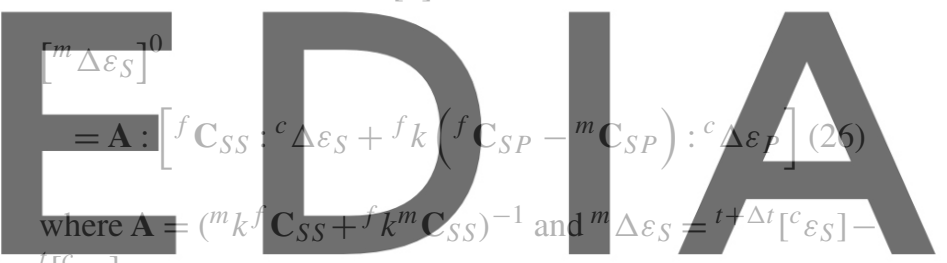

$\left[{ }^{c} \varepsilon_{S}\right]$.

The initial prediction of the serial strains, is obtained condowinload therversign owithabt the wateripark

alent to the serial stiffness of both materials. Once having made the strain prediction, fiber serial strains are obtained, for this first iteration and for iteration $n$, by using (22):

$t+\Delta t\left[{ }^{f} \Delta \varepsilon_{S}\right]^{n}={\frac{1}{f_{k}}}^{t+\Delta t}\left[{ }^{c} \varepsilon_{S}\right]-{\frac{}{{ }^{m} k}}_{f_{k}} t+\Delta t\left[{ }^{m} \varepsilon_{S}\right]^{n}$

where ${ }^{t+\Delta t}\left[{ }^{m} \varepsilon_{S}\right]^{n}={ }^{t}\left[{ }^{m} \varepsilon_{S}\right]+\left[{ }^{m} \Delta \varepsilon_{S}\right]^{n}$.

The following step consist in recombining the serial and parallel components of the strain tensor (18). In order to apply the constitutive equations to obtain the stress tensor and the updated internal variables for both materials. The fiber and matrix materials are modeled according to their own constitutive laws. In the case of an additive plasticity law, (23) is used to obtain the stress tensor for each one of them.

The serial stresses obtained for fiber and matrix from their constitutive law must fulfill iso-stress condition:

$\left[\Delta \sigma_{S}\right]^{n}={ }^{t+\Delta t}\left[{ }^{m} \sigma_{S}\right]^{n}-{ }^{t+\Delta t}\left[{ }^{f} \sigma_{S}\right]^{n} \leq$ tolerance

If the residual stress is smaller than the defined tolerance, the computed strains and stresses are considered correct and 
Fig. 5 Flow chart of the serial/parallel rule of mixtures algorithm
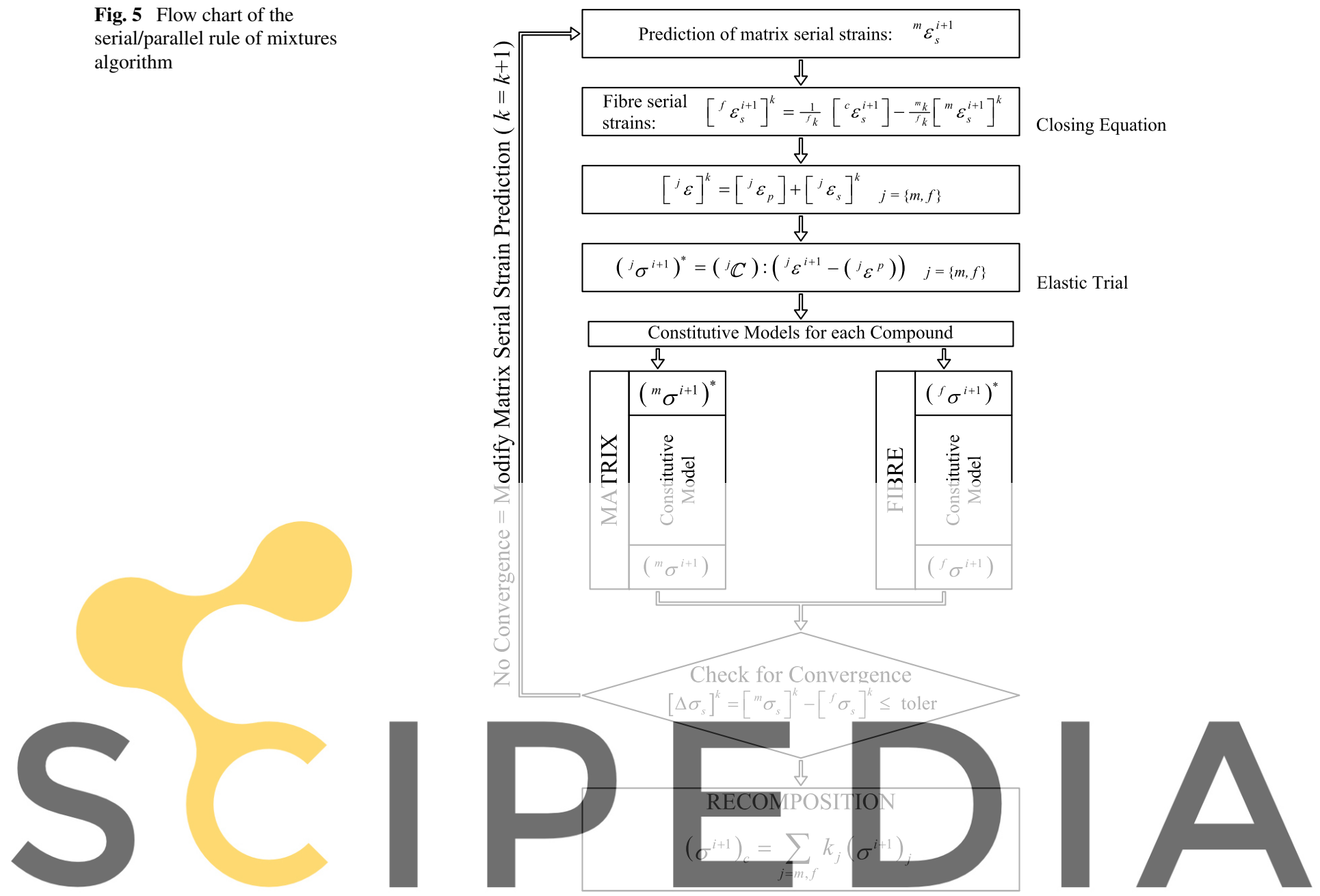

\section{Register for free at https//www. scipedia.com to} not fulfilled, the initial prediction of the matrix strain tensor has to be corrected. This correction is performed using a Newton-Raphson scheme, which is updated using the Jacobian of the residual forces. The Jacobian is obtained deriving the residue function with respect to the unknown. According to Rastellini [37], the expression for the Jacobian is given as follows:

$\mathbf{J}=\left.\frac{\partial \Delta \sigma_{S}}{\partial^{m} \varepsilon_{S}}\right|_{{ }^{m} \varepsilon_{S}={ }^{t+\Delta t}\left[{ }^{m} \varepsilon_{S}\right]^{n}}=\left[{ }^{m} \mathbf{C}_{S S}\right]^{n}+\frac{{ }^{m} k}{f_{k}}\left[{ }^{f} \mathbf{C}_{S S}\right]^{n}$

and, the expression for correcting the matrix serial strains becomes:

${ }^{t+\Delta t}\left[{ }^{m} \varepsilon_{S}\right]^{n+1}={ }^{t+\Delta t}\left[{ }^{m} \varepsilon_{S}\right]^{n}-\mathbf{J}^{-1}:\left[\Delta \sigma_{S}\right]^{n}$

The Jacobian must be obtained using the tangent constitutive tensor for the fibers and the matrix, in order to reach quadratic convergence in the serial/parallel mixing theory. However, depending on the constitutive equation defined for each material, it is not always possible to obtain an analytical expression for this tensor. In order to obtain a reliable download the version without the watermark a numerical derivation, using a perturbation method. A detailed description of the perturbation method used can be found in $[38,39]$.

\subsubsection{Implementation in a Finite Element Code}

The serial/parallel mixing theory is implemented in PLCd [40] finite element code. This code works with two and three-dimensional solid geometries and with beam elements. It can deal with kinematic and material nonlinearities. It uses various constitutive laws to predict the material behavior (Elastic, visco-elastic, damage, damageplasticity, etc. [41]) and uses different yield surfaces to control their evolution (Von-Mises, Mohr-Coulomb, improved Mohr-Coulomb, Drucker-Prager, etc. [28, 42]). The Newmark method [43] is used to perform dynamic analysis. A more detailed description of the code can be obtained from [44, 45].

The serial/parallel mixing theory is implemented in PLCd code at the constitutive level, adding the iterative 


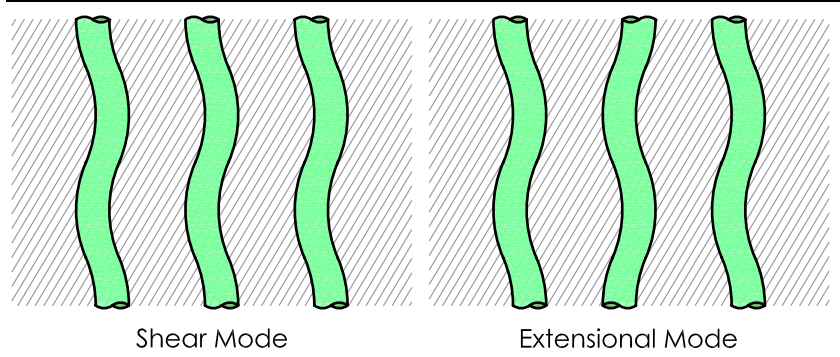

Fig. 6 Shear and extensional buckling modes defined by [1]

procedure described previously. This implementation is described in the flow chart shown in Fig. 5. The capability of PLCd to solve composite structures using the serial/parallel mixing theory has been already proven by Martinez et al. in [38].

\section{Compression Strength of Composite Materials}

Since the first investigation made by Rosen [1], many authors have studied the problem of long fiber composites under compression efforts. These studies have led to different formulations that provide an approximation to the maximum

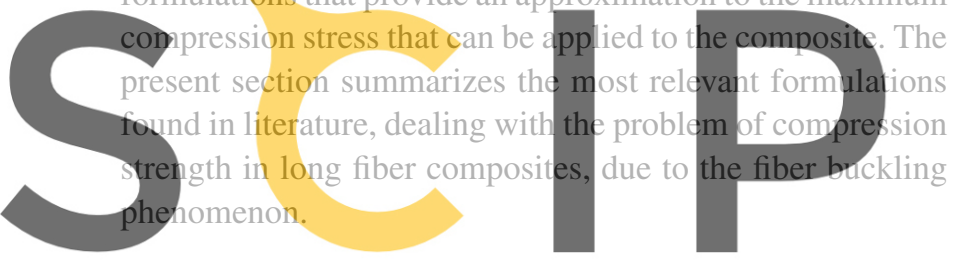

3.1 Rosen Model

Register for free at https//www.scipedia.com to

The first known study in which the compression strength of long fiber composites was related to the fiber buckling problem corresponds to Rosen [1]. This study has been summarized by Jones in [46]. To obtain the compression strength of the composite, Rosen considered the fiber as a column on an elastic foundation. With this model in mind, two different buckling modes were defined: extensional and shear buckling. These two modes are shown in Fig. 6.

To obtain the compression strength of the composite, Rosen defined a two dimensional model in which fibers are considered plates infinitely wide. With this assumption, an energy method [47] was applied to obtain the buckling load of the plate considering $m$ transversal restrictions along its length. Minimizing the resulting expression in function of $m$, the equations obtained to compute the composite maximum compression stress are, for each buckling mode:

- Extensional mode:

$$
\sigma_{c, \max }=2\left[V_{f}+\left(1-V_{f}\right) \frac{E_{m}}{E_{f}}\right] \sqrt{\frac{V_{f} E_{m} E_{f}}{3\left(1-V_{f}\right)}}
$$

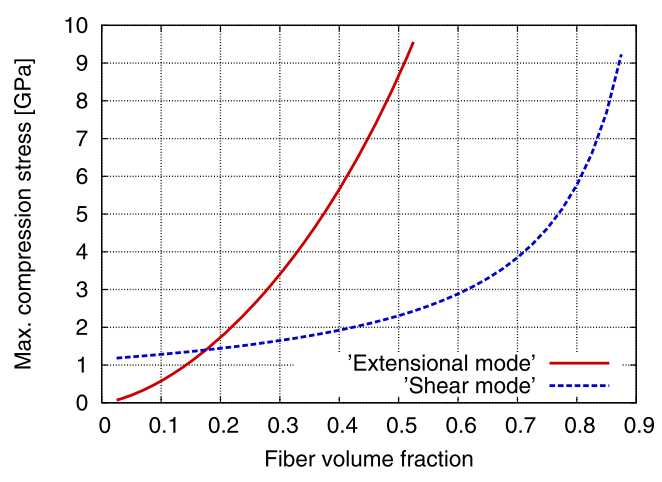

Fig. 7 Maximum compression stress that can be applied to an E-Glass composite using Rosen's formulation

being $V_{f}$ the fiber volumetric particination and $E_{m}$ and $E_{f}$ the matrix and fiber elastic modulus, respectively. This equation considers an iso-strain condition in fiber direction. If $E_{m} \ll E_{f}$, matrix stresses can be neglected and expression (31) can be simplified to:

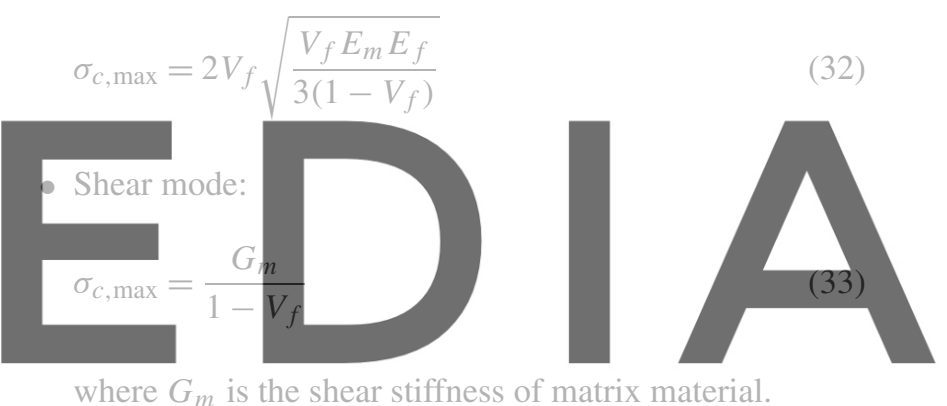

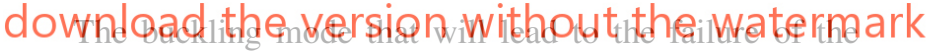

composite depends on the volume content of fiber in the composite. The plot of the results obtained with expressions (31) and (33) in function of the fiber volumetric participation for an E-Glass composite, is displayed in Fig. 7. This figure shows that for fiber volume contents lower than 0.18 the buckling mode that will lead to the composite failure is the extensional mode and, for fiber contents larger than 0.18 , the compression strength is produced by the shear mode.

The most known expression known of the Rosen model corresponds to the maximum compression of the composite due to shear buckling (33). This is because the fiber volume content in the most commonly used composites, is in the range of $50-60 \%$. It is easy to see, and Fig. 7 proves it for an E-Glass composite, that shear buckling is the mode that provides the lower compression strengths for these ranges of fiber volumetric participation.

Following the same idea used by Rosen to formulate his model, Lo-Chim [48] and Xu-Reifsnider [49] developed two different models that try to improve Rosen's one. Hereafter are described both of them. 


\subsubsection{Lo-Chim Model}

The model proposed by these authors follows the same procedure used by Rosen, reducing the number of assumptions made. These authors added a term, during the derivation of the result, to account for unknown boundary conditions. This term is obtained by fitting the model to the experimental results. Therefore, the equation proposed to obtain the compression strength of the composite is [48]:

$\sigma_{c, \max }=\frac{G_{12}}{1.5+12\left(\frac{6}{\pi}\right)^{2}\left(\frac{G_{12}}{E_{11}}\right)}$

where the variables $G_{12}$ and $E_{11}$ are obtained using the elastic mechanical parameters of fiber and matrix and their volumetric participation in the composite.

\subsubsection{Xu-Reifsnider Model}

The model proposed by Xu-Reifsnider [49] replace the Timoshenko beam formulation used to obtain the compression strength of a column, by the problem of a column embedded in an elastic foundation. With this substitution, the expression obtained by these authors to characterize the maximum strength that can be applied to the composite becomes:

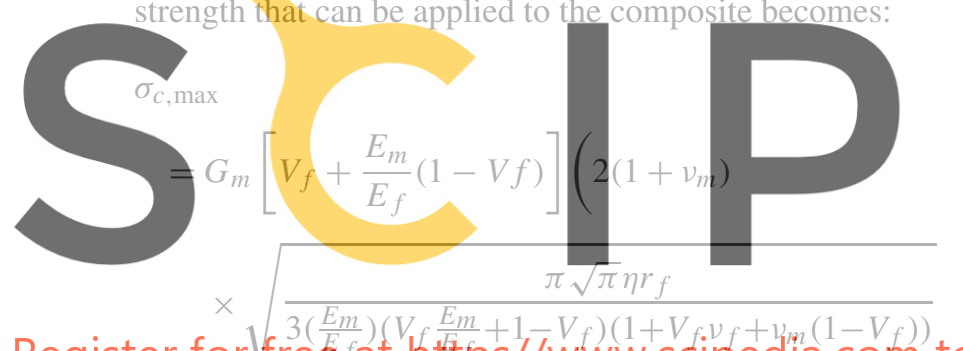

Register forlfreefat https//Www.scipedia.com to

$$
\left.+1-\xi-\frac{\sin \pi \xi}{2 \pi}\right)
$$

Being $\eta$ and $\xi$ two empirical parameters. These are introduced into the equation to consider the bond strength between fiber and matrix and the effect of matrix slippage, respectively.

From the first model developed by Rosen, and the updates proposed by Lo-Chim and Xu-Reifsnider, different authors have studied the fiber buckling problem in composites, obtaining more accurate expressions to compute the maximum compression strength that can be applied to the composite.

All new formulations maintain the dependence of fiber buckling in fiber volume fraction and matrix shear stiffness but they do also introduce a new parameter in the equation: fiber initial misalignments.

\subsection{Fiber Initial Misalignments}

The effect of fiber initial imperfections on the compression performance of long fiber composites has been assumed and proved by all authors studying the problem. Therefore, the measurement of fiber initial misalignments appears essential to obtain an accurate prediction of the compression strength of the composite [15].

Yurgartis [50] developed an optical technique consisting in cutting the composite at an angle and measuring the major axis of the ellipse formed by the intersection of a cylindrical fiber with the cutting plane. The determination of the cutting angle was made assuming a symmetric distribution of fiber misalignment. This technique has been refined afterwards by Barbero and Tomblin [3], which accurately measured the angle of cut by measuring the major and minor axes of the ellipse formed by the rod specimen.

A first attempt to obtain a three dimensional measurement of fiber initial misalignments was made by Paluch [51], by taking a stack of sections of the composite and matching the fibers found in each $X Y$ image of each section. From this first attempt, Clarke et al. [52] developed a new technique in which the three dimensional spatial distribution of fiber misalignments could be obtained. This technique uses a confocal laser scanning microscope (CLSM) to obtain the each fiber position along the $Z$-axis of the sample. The main advantage of using a CLSM is that the data acquisition is non-destructive, therefore the composite does not have to be

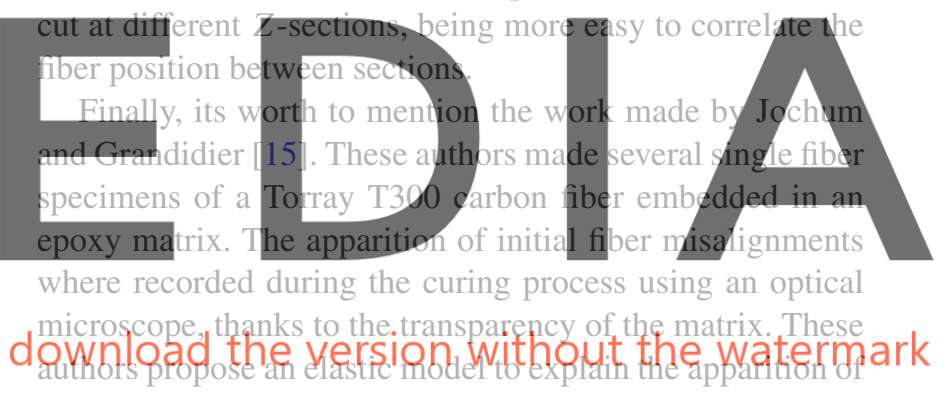

fiber initial misalignments during the curing process of the composite.

\subsection{Barbero and Tomblin Model}

Being aware of the importance of fiber initial misalignments in the compression performance of composite materials, Barbero and Tomblin [3, 4, 6] developed a formulation, based on continuous damage mechanics, to predict the compression strength of composite materials, taking into account fiber initial imperfections.

The model developed is based in the following hypothesis:

i. Matrix shear stiffness is non-linear and can be characterized by an hyperbolic tangent function.

ii. Fiber misalignments in the composite follow a Gaussian distribution.

Taking into account these two hypothesis, the relation between the buckling stress in the composite and the fiber imperfection is obtained using the principle of total potential 
energy. The final expression obtained to calculate the critical compression stress in the composite is [6]:

$$
\begin{aligned}
& \sigma(\alpha, \gamma) \\
& =\frac{\tau_{u}}{2(\gamma+\alpha)} \\
& \quad \times \frac{(\sqrt{2}-1)\left(e^{\sqrt{2} g}-e^{2 g}\right)+(\sqrt{2}+1)\left(e^{(2+\sqrt{2}) g}-1\right)}{1+e^{2 g}+e^{\sqrt{2} g}+e^{(2+\sqrt{2}) g}}
\end{aligned}
$$

With $g=\gamma G_{L T} / \tau_{u}$. And being $\tau_{u}$ the maximum shear strength of matrix material, $G_{L T}$ the matrix shear stiffness (defined by an hyperbolic tangent function), $\gamma$ the shear strain in the composite and $\alpha$ the misalignment angle of fibers.

Expression (36) provides the maximum compression strength that can be applied to the composite considering that fibers have a misalignment angle $\alpha$. Once having this expression, a continuum damage model is applied to the compression stress of the composite to obtain the composite performance. Therefore,

$\sigma_{C}=\sigma(\alpha)[1-\omega(\alpha)]$

Where $\omega(\alpha)$ is the area of buckled fibers per unit of initial fiber area. The area of bucked fibers is proportional to the area under the normal distribution located beyond the misalignment angle $\pm \alpha$, and it can be computed as,

$\omega(\alpha)=2 \int_{\alpha}^{\infty} f\left(\alpha^{\prime}\right) \mathrm{d} \alpha^{\prime}$

with $f(\alpha)$ the probability density of the normal distribution.

Thus, the model developed by Barbero and Tomblin not only provides the maximum compression strength that can be applied to the composite before fiber buckling (for a given misalignment angle, (36)), but also provides the stress evolution of the composite. This stress evolution depends on the number of fibers that have buckled; fibers with large misalignments will buckle for lower loads. Equation (37) is important because there are few models that provide the postcritical performance of the composite once the maximum compression stress has been reached.

\subsection{Balacó de Morais Model}

Another approximation to the compression strength of composite materials due to fiber microbuckling is the one provided by Balacó de Morais [7-9]. This author considers that fiber misalignments have a sinusoidal shape and assumes a shear buckling mode of the fibers. The compression strength is derived from the variational principle of the minimum potential energy of the system, which is defined as:

$\Pi=U_{f}+U_{m}-W$
Being $U_{f}$ the fiber bending strain energy, $U_{m}$ the matrix shear strain energy and $W$ the work done by the compression load. To obtain the shear strain energy of matrix material a Drucker-Prager yield model has been considered. The work done by the compression load is obtained using a large displacement formulation.

Once having defined all parameters of (39), the minimum potential energy equation is numerically solved for successive increments of the stress acting on the fiber, to obtain the lamina compression strength using the parallel mixing theory:

$\sigma_{L}=V_{f} \sigma_{f}+\left(1-V_{f}\right) \sigma_{m}$

The main problem with this approach is that it is computationally very expensive. To solve this inconvenience, the model is simplified to a linear formulation [9]. The new model assumes small displacements to compute the work done by the compression load, matrix strain energy is obtained considering linear elasticity and fiber strain energy is neglected. With these assumptions, a fourth order polynomial equation is obtained that provides the compression strength of the composite.

A further simplification is made with the consideration that if fibers are considerably stiffer than matrix, when matrix fails, matrix compression stress is much smaller than the compression yield stress and thus negligible. This assumption leads to a close form expression in which the composite compression strength is obtained as:

$\sigma_{C}=\frac{\tau_{u m}}{\theta_{0}+\frac{1-V_{f}}{1+V f} \frac{\tau_{u m}}{G_{m}}}$

Being, $\tau_{\text {um }}$ the maximum shear stress that can take the matrix, $\theta_{0}$ the initial fiber misalignment angle and $G_{m}$ the matrix shear stiffness. According to Balacó de Morais [9], this expression works well with composites made with stiff fibers such as Kevlar or Carbon but loses its accuracy with less stiff fibers such as E-Glass.

\subsection{Drapier Model}

An interesting approach to obtain the compression strength of composite materials is the one developed by Drapier et al. $[10,11]$, who solve the fiber microbuckling problem in a two scale context. In it, the displacement field is formulated taking into account the meso displacements of the structure and the micro displacements of the fibers due to their initial misalignments.

Drapier model appears as a response to the experimental results described by Wisnom [53] and Grandidier et al. [54]. These authors showed that the compression performance of the composite depends, not only on the material 


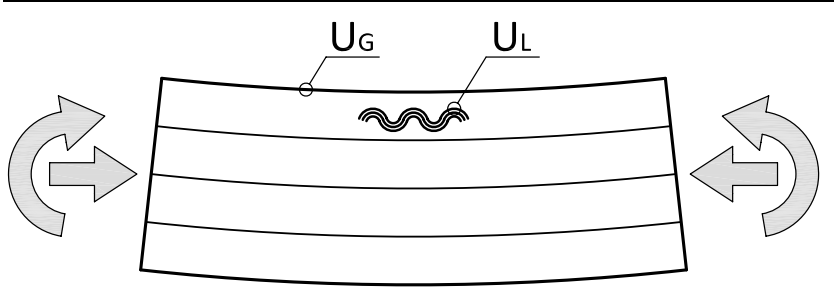

Fig. 8 Deformations defined in the meso-micro model defined by Drapier et al. Figure obtained from [11]

and its initial imperfections, but also on the meso-scale configuration of the composite (composite thickness and stacking sequence) and the loads applied to it. In order to take into account the micro and the meso scales, the model developed by Drapier defines the composite displacement as the sum of the composite meso, or global, displacement and the fibers micro, or local, displacement:

$u(x)=u_{G}(x)+u_{L}(x)$

The physical meaning of these two displacements is shown in Fig. 8, where both displacements are represented. This figure has been obtained from [11].

Once having defined the two scales that are used to characterize the composite, the authors develop a specific finite element which formulation is obtained by coupling the equilibrium in both scales. This element is defined using a twodimensional characterization of the composite. The element equilibrium is written using the Principle of Virtual Work (PVW), and its expression is:

$\int_{\omega}\left\{{ }^{f} V E_{f} r_{g}^{2} v^{\prime \prime} \delta v^{\prime \prime}+C^{T} \delta \gamma\right\} d \omega+\langle F ; \delta u\rangle=0$

where ${ }^{f} V$ is the volume fraction of fibers, $E_{f}$ is fiber young modulus and $r_{g}$ the radii of gyration of fibers. The parameter $v$ corresponds to the vertical component of the total displacement $u$, and $\gamma$ is the Green-Lagrange strain tensor. Finally, $C^{T}$ stands for the secant stiffness matrix of the composite and $F$ for the external forces applied to the element.

The first term of the PVW defined by (43), represents the fiber bending stiffness and is necessary to obtain a good prediction of the compression strength of the composite [11].

The model is defined considering the fibers an elastic material and modeling matrix material as non-linear, with a J2 law.

Drapier et al. compare the results obtained with the model with theoretical and experimental data. Special attention is paid to the capacity of the model to take into account the stacking sequence of the laminate, its thickness and the type of loading and boundary conditions. These comparisons show the good performance of the model.
3.6 Other Authors Dealing with the Problem. Structural Models

Another scope used to solve the problem, different to the formulations shown above, is the one used by Parnes and Chiskis [12] or Akbarov and Kosker [13, 14]. These authors, solve the problem studying the structural compatibility between fiber and matrix stress-strain fields.

\subsubsection{Parnes and Chiskis Model}

The formulation proposed by Parnes and Chiskis [12] is based on the study of a two dimensional representative volume element of the composite, where fibers are characterized as periodically spaced layers embedded in an elastic matrix. Fibers displacement is defined with a sinusoidal function. The authors apply the Euler-Bernoulli elastic theory to this RVE to obtain the composite stresses and strains. To do so, they take into account the structural compatibility between fiber sinusoidal displacement and matrix restrain.

With the developed formulation, the value of fibers strains are:

$\varepsilon_{S, T}=\frac{1}{1+{ }^{m} v} \frac{{ }^{m} E}{f^{f} E}\left[2+\frac{1+\frac{1}{1-2^{m} v}}{\eta_{F}} \Psi_{S, T}\right]+\frac{\eta_{F}{ }^{2}}{3}$

with, $\eta_{F}=\pi \frac{H_{F}}{L}$ ( $H_{F}$ representing the fiber thickness and $L$ the buckling length) and $\Psi_{S, T}$ a function of the RVE cell geometry and the matrix Poisson modulus. Two different $\Psi$ functions are defined, $\Psi_{S}$ and $\Psi_{T}$, to characterize shear and transverse buckling modes, respectively.

The buckling stress is obtained minimizing the value of $\varepsilon_{S, T}$ in function of $\eta_{F}$, that is equivalent to minimize $\varepsilon_{S, T}$ according to the buckling length $L$.

In case of having a composite with low fiber volumetric participation, the authors prove that the critical buckling strain does not depend on the buckling mode and its value can be approximated by:

$\varepsilon_{c r} \approx\left[\frac{1}{3}\left(\frac{\left(1+{ }^{m} v\right)\left(3-4^{m} v\right)}{1-{ }^{m} v}\right) \frac{{ }^{f} E}{{ }^{m} E}\right]^{-2 / 3}$

With the expressions obtained, the authors conclude that elastic fiber buckling of composites takes place specially in composites with small fiber volume fractions and high ${ }^{f} E /{ }^{m} E$ ratios. They also conclude that buckling only takes place in transverse mode, as the buckling stresses obtained for the shear mode are always larger.

Finally, the study of the results obtained with the developed formulation provides the authors with a function $v_{f}^{*}$ that provides a differential behaviour of the composite according to its fiber volumetric participation. Buckling will take place with a finite wave-length if fiber volumetric participation in the composite is lower than $v_{f}^{*}$, and will buckle 
with an infinite wave-length if the volumetric participation is larger.

\subsubsection{Akbarov and Kosker Model}

The solution of Akbarov and Kosker follows the path defined by Guz and Lapusta [55], based on using the three dimensional linearized equations of stability, and the model of a piecewise uniform medium. The matrix (uniform medium) is simulated with a viscoelastic model. Fibers are simulated as an elastic material with an initial periodic imperfection along its longitudinal axis.

Based on the viscoelastic formulation defined for the matrix, the formulation developed calculates the time at which fibres buckle when the composite is loaded with a uniform pressure. In [13], the authors study the mechanical behaviour of a single fiber embedded in an infinite matrix. In [14], what is studied is the interaction of two adjacent fibres under the same conditions. In both studies, fiber buckling is considered to occur when the growth of the fiber initial imperfections increase indefinitely.

\subsection{Puig and Oller Model}

The model developed by Puig and Oller $[16,56]$ to obtain the compression strength of composite materials due to fiber buckling can be considered the point of departure for the formulation that is presented in this paper.

These authors deal with the compression strength problem using a continuum mechanics approach and obtaining the constitutive performance of the composite using the parallel mixing theory.

Up to this point, all authors studying the fiber microbuckling problem obtain expressions that provide the maximum compression stress that can be applied to the composite, which is treated as a single material. These formulations cannot provide solutions for composites in which its constituent materials show a non-linear behavior or, when nonlinearities are considered, they are pre-defined and are part of the formulation.

The main advantage of the model proposed by Puig and Oller is that, using the mixing theory, composite constituents can be simulated with any existing constitutive model. This increases the applicability of the formulation developed, being possible to use it for simulating any possible composite in which the compression strength due to fiber buckling has to be taken into account.

The formulation proposed by these two authors is a modification of the isotropic damage model defined by Kachanov $[57,58]$, that adds a new internal variable related to the fiber buckling phenomenon. With the proposed formulation, the stresses in the fibers are:

$\mathbf{f}_{\sigma}=\left(1-d_{f}\right)\left(1-d_{p}\right) \mathbf{C}: \varepsilon$ being $\mathbf{C}$ the composite constitutive tensor, $d_{f}$ the mechanical damage parameter and $d_{p}$ the buckling damage parameter. The expression for this last parameter is:

$d_{p}=f_{1}$ (fiber) $f_{2}$ (matrix)

Where $f_{1}$ (fiber) is a function that depends on fiber buckling stress and $f_{2}$ (matrix) is the matrix damage parameter. Function $f_{1}$ is obtained from the critical Euler load that can be applied to a column. Its final expression is [59]:

$f_{1}($ fiber $)=\left(\frac{4 l}{n \pi d}\right)^{2} \varepsilon$

Being $l$ the length of the fiber, which is a parameter required by the formulation, $n$ the buckling mode, $d$ the fiber diameter and $\varepsilon$ the longitudinal strain of fiber material. The buckling mode that will lead to fiber buckling is computed as:

$n=\frac{4 l}{\pi d} \sqrt{\varepsilon}$

Although the model developed by Puig and Oller provides a good numerical performance, it fails in not considering important parameters related to the fiber microbuckling problem such as fiber initial misalignments. Also, with the proposed formulation, the effect of matrix restrain over fibers is included into the formulation in a too simplistic form, with the matrix damage parameter, $f_{2}$.

\section{Homogenized Constitutive Equations to be Introduced into the Serial/Parallel Mixing Theory: CuBER Problem}

\subsection{Representative Volume Element to Solve the Fiber Buckling Problem}

According to the different authors that have studied the problem, a micro-model capable to simulate the fiber buckling phenomenon must take into account fiber initial misalignments, the proportion of fiber and matrix in the composite and matrix shear strength. A Representative Volume Element (RVE), depending on these three parameters, can be the one shown in Fig. 9. This figure also shows, in dashed lines, the final position expected in the fibers after applying the compression force.

The performance that is obtained from fiber and matrix materials, when solving the RVE shown in Fig. 9, must be translated to constitutive properties in order to include them in a mixing theory formulation. Figure 9 shows that, when the composite is compressed, fiber initial misalignments tends to increase; but their deformation is restrained by matrix material. Thus, the constitutive performance expected in each material is: 


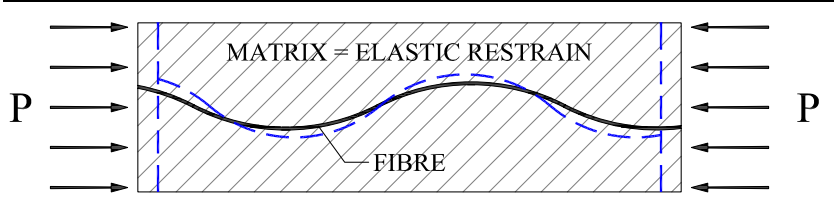

Fig. 9 Fiber-matrix system. Fiber behaviour when the composite is compressed

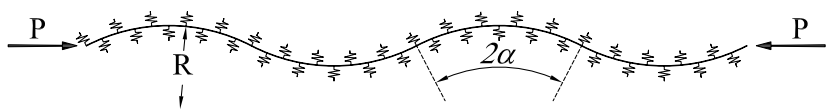

Fig. 10 Curved bar under unilateral elastic restrain problem

Fiber: Its structural stiffness is reduced due to its original misalignment, as a result of its structural deformation.

Matrix: Stresses in it increase because of the restriction made over fiber movement.

These two performances can be obtained also by solving a simplified unidirectional model consisting in a Curved Bar under unilateral Elastic Restrain (CuBER), like the one shown in Fig. 10. The solution to the structure shown in this figure has already been used by other authors to solve similar problems. Naik et al. [60] used it to study weave fabric composites and Wang and Shenoi [61] to study curved sandwich beams.

\subsection{CuBER Equations}

The problem of a curved bar under unilateral elastic restrain was first formulated and solved by Hetenyi [62]. Figure 11 shows the geometry considered.

The hypothesis in which the curved bar under unilateral restriction problem is solved are the following:

i. All components defining the problem, the bar and the foundation in which it is supported, have an elastic behavior.

ii. The axis of the bar is contained in an arch of a circle of radius $R$. The arch length is defined by its internal angle $2 \alpha$ (Fig. 11).

iii. The reaction forces of the foundation are proportional to the radial deflection of the bar.

iv. The deformation of the bar due to normal stresses is negligible.

According to the hypothesis exposed, the elastic restrain of the bar can be idealized as a continuous spring which produces a radial load in the bar, proportional to its radial displacement:

$q=\mathrm{k} \omega$

Being $q$ the radial load produced by the elastic restriction over the bar, $\omega$ the bar radial displacement and $\mathrm{k}$ the elastic restrain.

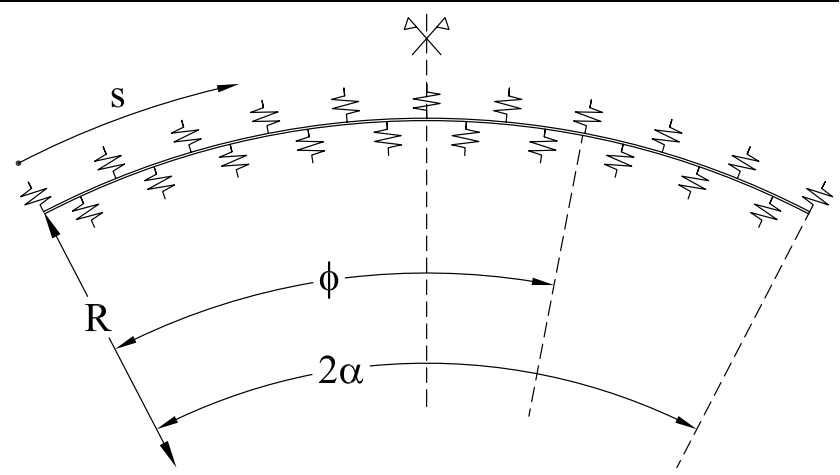

Fig. 11 Geometry of the bar to be considered in the CuBER problem

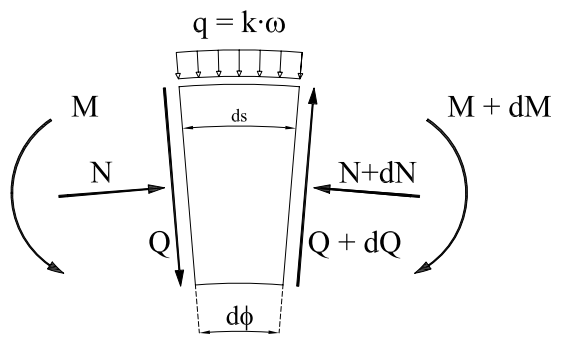

Fig. 12 Forces acting on an infinitesimal section of the curved bar

The equations describing the bar behavior are obtained studying an infinitesimal section of the bar, such the one displayed in Fig. 11, in which acts a normal force $N$, a shear force $Q$ and a bending moment $M$. These are displayed in Fig. 12.

The study of the equilibrium of forces in an infinitesimal section of the beam leads to three different equilibrium equations. The equilibrium equation in the radial direction can be written as:

$q d s-N d \phi=d Q$

The equilibrium equation in the tangential direction is:

$Q d \phi=d N$

And the momentum equilibrium equation is written as:

$Q R d \phi=d M$

Neglecting the axial deformation of the bar due to the normal force $N$ (hypothesis iv), the differential bending equation of a circular arch of radius of curvature $R$ and flexural rigidity $E I$ is, according to Hetenyi [62],

$E I\left(\frac{d^{2} \omega}{d s^{2}}-\frac{\omega}{R^{2}}\right)=-M$ 
Rearranging (51) to (54), the bar displacement in its radial direction is defined by the differential equation:

$\frac{d^{5} \omega}{d \phi^{5}}+2 \frac{d^{3} \omega}{d \phi^{3}}+\eta^{2} \frac{d \omega}{d \phi}=0$

where,

$\eta=\sqrt{\frac{R^{4} \cdot \mathrm{k}}{E I}+1}$

The general solution for (55) is:

$$
\begin{aligned}
\omega(\phi)= & C_{0}+\left[C_{1} \cosh \left(\eta_{1} \phi\right)+C_{2} \sinh \left(\eta_{1} \phi\right)\right] \cos \left(\eta_{2} \phi\right) \\
& +\left[C_{3} \cosh \left(\eta_{1} \phi\right)+C_{4} \sinh \left(\eta_{1} \phi\right)\right] \sin \left(\eta_{2} \phi\right)
\end{aligned}
$$

with,

$\eta_{1}=\sqrt{\frac{\eta-1}{2}}, \quad \eta_{2}=\sqrt{\frac{\eta+1}{2}}$

and $C_{0}, C_{1}, C_{2}, C_{3}, C_{4}$ the integration constants of the differential equation, which value is obtained when imposing the boundary conditions of the problem.

Two of these integration constants can be obtained straightforward using the symmetry shown in Fig. 11. It can be easily verified that, if the origin of angle $\phi$ is defined in the axis of symmetry, the following boundary conditions must be fulfilled:

$\left.\frac{d \omega}{d \phi}\right|_{\phi=0}=0 \quad$ and $\quad Q(\phi=0)=0$

The result of applying these two conditions to the previous equations is that the integration constants $C_{2}$ and $C_{3}$ have to be 0.0 .

If integration constants $C_{2}$ and $C_{3}$ are eliminated, the general expression of the beam displacement becomes:

$$
\begin{aligned}
\omega(\phi)= & C_{0}+C_{1} \cosh \left(\eta_{1} \phi\right) \cos \left(\eta_{2} \phi\right) \\
& +C_{4} \sinh \left(\eta_{1} \phi\right) \sin \left(\eta_{2} \phi\right)
\end{aligned}
$$

and, replacing (60) into (51) to (54), the efforts on the bar are:

$$
\begin{aligned}
M(\phi)= & -\frac{E I}{R^{2}}\left\{C_{0}-2 \eta_{1} \eta_{2}\left[+C_{1} \sinh \left(\eta_{1} \phi\right) \sin \left(\eta_{2} \phi\right)\right.\right. \\
& \left.\left.-C_{4} \cosh \left(\eta_{1} \phi\right) \cos \left(\eta_{2} \phi\right)\right]\right\} \\
Q(\phi)= & -\frac{E I}{R^{3}} 2 \eta_{1} \eta_{2}\left\{\left(\eta_{1} C_{1}+\eta_{2} C_{4}\right) \cosh \left(\eta_{1} \phi\right) \sin \left(\eta_{2} \phi\right)\right. \\
& \left.+\left(\eta_{2} C_{1}-\eta_{1} C_{4}\right) \sinh \left(\eta_{1} \phi\right) \cos \left(\eta_{2} \phi\right)\right\} \\
N(\phi)= & R \mathrm{k} C_{0}+\frac{E I}{R^{3}} 2 \eta_{1} \eta_{2}\left\{+C_{1} \sinh \left(\eta_{1} \phi\right) \sin \left(\eta_{2} \phi\right)\right. \\
& \left.-C_{4} \cosh \left(\eta_{1} \phi\right) \cos \left(\eta_{2} \phi\right)\right\}
\end{aligned}
$$

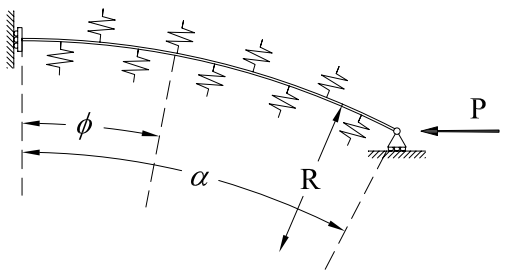

Fig. 13 Boundary conditions to be applied to the CuBER problem

According to Hetenyi [62], the angular deflection of the beam can be obtained as the integral of the elementary rotations $M / E I$ along the bar. If this integral starts in the axis of symmetry shown in Fig. 11, the initial rotation is zero and the value of the angular deflection becomes:

$\theta(\phi)=\int_{0}^{S} \frac{M(s)}{E I} d s=\int_{0}^{\phi} \frac{M(\varphi)}{E I} R \mathrm{~d} \varphi$

And the vertical and horizontal displacement of the beam can be obtained as,

$u(\phi)=u_{0}-\int_{0}^{\phi} \theta(\varphi) R \sin (\varphi) \mathrm{d} \varphi$
$v(\phi)=v_{0}-\int_{0}^{\phi} \theta(\varphi) R \cos (\varphi) \mathrm{d} \varphi$

with,

$\omega=u(\phi) \sin (\phi)+v(\phi) \cos (\phi)$

The expression for the bar displacements $\omega, u$ and $v$, as well as for the bar efforts $M, Q$ and $N$, depends on the value of the integration constants: $C_{0}, C_{1}$ and $C_{4}$. The value of these constants is obtained by defining three more boundary conditions of the problem.

\subsection{Boundary Conditions and Solution of the CuBER Problem}

The final expressions defining the behavior of the CuBER problem require the definition of three boundary conditions, in order to obtain the value of the integration constants $C_{0}$, $C_{1}$ and $C_{4}$.

Using all symmetries existing in the geometry shown in Fig. 10, the problem can be simplified to the one shown in Fig. 13.

In which the boundary conditions to be applied are:

1st BC: $\quad M(\phi=\alpha)=0$

2nd BC: $\quad N(\phi=\alpha) \cos (\alpha)-Q(\phi=\alpha) \sin (\alpha)=P$

3rd BC: $v(\phi=\alpha)=0$

These three boundary conditions, applied to (60), (61) and (63), define a linear system of equations that will provide the specific values of $C_{0}, C_{1}$ and $C_{4}$. 
4.4 Definition of the CuBER Problem Using the

Composite Properties

This section describes the properties of the composite required to have well defined the CuBER problem.

The fiber buckling problem is formulated for composite materials made of long fibers embedded in a matrix. Thus, the fiber buckling problem is characterized by the properties of fiber and matrix materials. If the composite is a laminate, the described properties are required for each layer.

\subsubsection{Matrix Characteristics}

When solving the CuBER problem, matrix is considered an isotropic material. This supposition allows defining the elastic restrain $\mathrm{k}$ defined in (50), as a constant variable with the same value in all space directions.

Although the serial/parallel mixing theory can use any type of constitutive law to characterize the materials that compose the composite, the formulation developed to solve the fiber-buckling phenomenon uses the isotropic damage formulation described in $[58,63]$ to simulate matrix material. With this formulation, stresses in matrix are obtained as:

${ }^{m} \boldsymbol{\sigma}=(1-d)^{m} \boldsymbol{\sigma}_{0}$

Being $\sigma$ the damaged (or real) stress tensor and $\sigma_{0}$ the effective stress tensor and $d$ the damage parameter. In an isotropic damage formulation, the material stiffness is also affected by the damage parameter. Therefore, the restrain that is applied by matrix to the fiber is computed as:

$\mathrm{k}=(1-d) \mathrm{k}_{0}$

Where $\mathrm{k}_{0}$ corresponds to the initial elastic restrain, which value is defined as the matrix Young Modulus, ${ }^{m} E$, and $\mathrm{k}$ is the effective restrain that can be applied in function of the level of damage existing in matrix material.

Although the performance of matrix material has been defined using a damage formulation, in the procedure developed this formulation can be easily replaced by other material evolution laws such as plasticity. In the particular case of a plastic law, the internal variable $d$ used in (67) must be replaced by $\kappa$, being $\kappa$ the plastic damage variable.

\subsubsection{Fiber Characteristics}

The equations describing the CuBER problem require the elastic stiffness of fiber material. Usually, fiber buckling takes place before the material reaches its threshold stress so, if fiber is defined by its Young Modulus, in order to take into account possible damages in fiber material (i.e. if a tensile stress is applied to the composite before compressing

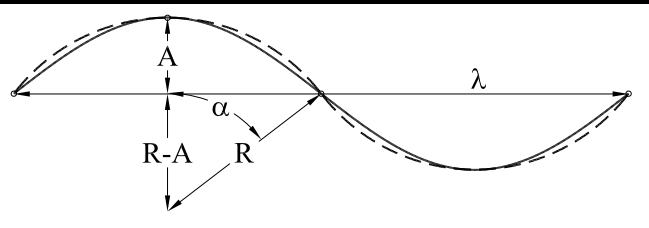

Fig. 14 Transformation of a sinusoidal shape into a circumferential one

it), the elastic stiffness used in the CuBER problem for fiber material is:

${ }^{f} E=(1-d)^{f} E_{0}$

Equation (68) assumes that fibers are simulated using a damage formulation. But, as has been already said for matrix, this parameter can be replaced by any other internal variable representing the stiffness reduction of the material, if a different material characterization is used.

\subsubsection{Fiber Misalignments}

Fiber misalignments are commonly defined in literature with a sinusoidal shape, characterized by its amplitude $(A)$ and wavelength $(\lambda)$. To be able to use the CuBER problem formulation, it is necessary to transform this shape into an arch of circumference. The formulation proposed considers that both curves are similar enough to be replaced one by the other (Fig. 14).

Applying the circumferential equation, the radius and the arch angle can be obtained from the amplitude and wavelength values as:

$$
\begin{aligned}
& R=\frac{A^{2}+(\lambda / 4)^{2}}{2 A} \\
& \alpha=\arcsin \left(\frac{\lambda}{4 R}\right)
\end{aligned}
$$

\subsubsection{Non-linear Performance of the CuBER Problem}

The CuBER problem is solved assuming that all components are elastic. However, both fiber and matrix materials, are simulated using a damage formulation, which provides a non-linear material behavior.

In order to be able to solve the CuBER problem formulation when any of the composite components reach its threshold stress, an explicit incremental approach is used to solve the CuBER equations. This approach is based on the assumption that during a load step, the variation in the stiffness of the composite components is small enough to be replaced by a constant stiffness value. The stiffness values considered at each time step for the matrix and the fiber are the ones exposed in (67) and (68), respectively.

Considering correct the assumption that the stiffness variation is negligible during a load step, what must be taken 
Fig. 15 Evolution of fiber misalignments in the loading process
Load step 2. Matrix already damaged $\rightarrow$ $\rightarrow$ Larger fibre deformation

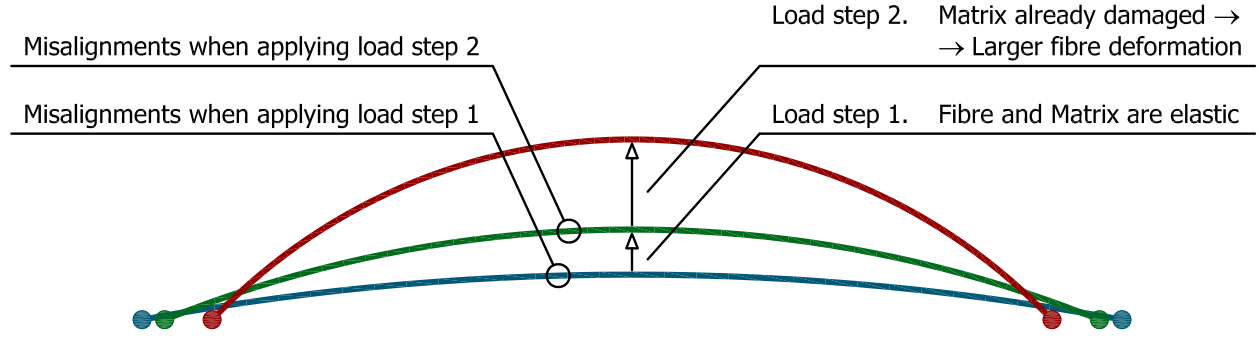

Total force applied: $2 \mathrm{P}$

Load step 1: $\Delta \mathrm{F}=\mathrm{P} \quad$ Load step 2: $\Delta \mathrm{F}=\mathrm{P}$

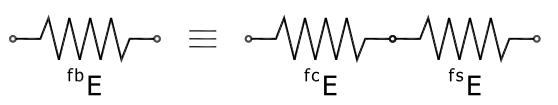

into account is the stiffness variation of both materials along the loading process. To do so, an incremental approach is used: At each load step, the fiber misalignment geometry is actualized according to the results obtained when solving the CuBER problem. This procedure requires that the load $P$, defined in (65), must be the load increment corresponding to the current load step, and not the total load applied to the element.

Applying this explicit incremental methodology, the fiber misalignments will vary according to the real material properties of the composite components, and the linear formulation of the CuBER problem is capable of solving a nonlinear problem. Figure 15 shows schematically the expected variation of fiber misalignments as the compression load increases in the composite.

\subsection{Fiber Homogenized Constitutive Equation}

The effects of fiber misalignment and its interaction with matrix material, obtained when solving the CuBER problem, must be included in the fiber constitutive equation so the serial/parallel mixing theory can take them into account. These effects are applied only to the longitudinal direction of the fiber.

The solution of the CuBER problem provides a structural displacement of fibers due to their initial misalignments. To include this effect into the constitutive equation of fiber material, this displacement must be transformed into a strain value. As the CuBER problem does not take into account the normal deformation of the bar due to the compression force [62], the total parallel strain in fiber material is divided in two components: a structural strain, ${ }^{f s} \varepsilon_{P}$, provided by the CuBER problem; and a constitutive strain, ${ }^{f c} \varepsilon_{P}$, due to the compression force applied to the composite. Thus, the total parallel strain in the fiber, ${ }^{f} \varepsilon_{P}$, is:

${ }^{f} \varepsilon_{P}={ }^{f c} \varepsilon_{P}+{ }^{f s} \varepsilon_{P}$

Equation (70) considers a serial distribution of the two mechanical phenomenons, constitutive and structural, that take place in the fiber material. Therefore, the effect of fiber misalignment introduces a new structural stiffness to the fiber material, as it is shown in the following diagram:
Fig. 16 Serial coupling of the fiber structural and constitutive stiffness

With the configuration shown in Fig. 16, the new fiber buckling stiffness of fiber material ${ }^{f b} E$, in its longitudinal direction, can be computed as the serial composition of the fiber constitutive $\left({ }^{f c} E\right)$ and structural $\left({ }^{f s} E\right)$ stiffness.

$\frac{1}{f^{b} E}=\frac{1}{f^{c} E}+\frac{1}{f^{s} E}$

The fiber constitutive stiffness corresponds to the Young Modulus of fiber material in its longitudinal direction, and fiber structural stiffness is obtained form the results provided when solving the CuBER problem.

The new fiber buckling stiffness, ${ }^{f b} E$, is introduced in the constitutive equation of fiber material using a damage formulation. Thus, a new fiber buckling parameter, ${ }^{f b} d$ is defined as:

${ }^{f b} E=\left(1-{ }^{f b} d\right)^{f c} E \rightarrow{ }^{f b} d=1-\frac{{ }^{f b} E}{{ }^{f c} E}$

The fiber buckling parameter affects the constitutive performance of fiber material reducing the effective strength of the material only in its longitudinal direction. Thus, the new stresses in fiber are obtained from the elastic prediction of the stresses as:

${ }^{f} \boldsymbol{\sigma}=\left(I-{ }^{f b} D\right) \cdot{ }^{f} \boldsymbol{\sigma}_{0}$

with,

${ }^{f b} D=\left[\begin{array}{cccc}{ }^{f b} d & 0 & \cdots & 0 \\ 0 & 0 & & 0 \\ \vdots & & \ddots & \vdots \\ 0 & 0 & \cdots & 0\end{array}\right]$

The fiber buckling formulation described above can be coupled easily with the constitutive damage formulation used to characterize fiber material. And, the final stress in 
fiber material, when coupling the effects of fiber buckling and the constitutive effects, is:

${ }^{f} \boldsymbol{\sigma}=(1-d) \cdot\left(I-{ }^{f b} D\right) \cdot{ }^{f} \boldsymbol{\sigma}_{0}$

Being $d$ the damage parameter obtained from the damage constitutive equation.

\subsubsection{Fiber Structural Stiffness, ${ }^{f s} E$}

The fiber structural stiffness that appears in (71) is obtained when solving the CuBER problem, from the relation between the force applied as a boundary condition and the displacement obtained as a result of this force. Because the problem is elastic, the relation between the displacement obtained when solving the CuBER problem and the force applied will be always the same. However, in order to apply a force proportional to the dimensions of the problem, the force $P$ that will be defined as a boundary condition correspond to the force corresponding to the total deformation found in the RVE to be solved. Being the total deformation of the element ${ }^{f} \varepsilon_{P}$, the stresses in the fiber will be:

$\sigma={ }^{f c} E \cdot{ }^{f} \varepsilon_{P}$

and, the force in one fiber:

$P=\sigma \cdot A_{f i b}$

Being, $A_{f i b}$ the area of one fiber of the composite. The force computed with this procedure is applied to the CuBER problem to obtain a longitudinal displacement of the fiber, $u(\phi=\alpha)$ (see Fig. 13). This displacement is transformed into a strain dividing by the total length of the bar considered in the CuBER problem. This length corresponds to one quarter of the wave-length (Fig. 13) and is defined as a misalignment property. Therefore, the strains in the element due to the structural displacement are,

$\varepsilon=\frac{u(\phi=\alpha)}{0.25 \lambda}$

and the structural stiffness can be obtained from the strainstress relation:

${ }^{f s} E=\frac{\sigma}{\varepsilon}=\frac{P}{A_{f i b}} \frac{0.25 \lambda}{u(\phi=\alpha)}$

Equation (79) shows that the structural stiffness varies in function of the relation between $P$ and $u$, which depends of fiber initial misalignments and the matrix stiffness. This relation remains constant while these two parameters remain constant but, as soon as fiber misalignments suffer a large variation or the matrix stiffness is reduced, the structural stiffness varies, modifying also the constitutive performance of fiber in the composite.
Once having the structural stiffness due to fiber misalignments, the real structural deformation can be computed using (70) and (71), obtaining:

${ }^{f s} \varepsilon=\frac{{ }^{f c} E}{f^{c} E+{ }^{f s} E} \cdot{ }^{f} \varepsilon$

Although the only parameter required to modify the fiber constitutive equation is the structural stiffness, it is important to obtain the real strains affected by the fiber buckling problem. These strains are required to obtain the real deformation of the fiber, in order to apply the incremental approach described in Sect. 4.4.4. When solving the CuBER problem, the variation obtained for fiber misalignments (amplitude and wave-length) correspond to an input strain of ${ }^{f} \varepsilon$, while the real strain that should be considered is ${ }^{f s} \varepsilon$. Thus, the values of the results obtained must be scaled by:

$\mathrm{SF}=\frac{{ }^{f s} \varepsilon}{f_{\varepsilon}}$

To obtain the new amplitude of fiber misalignments, the vertical displacement of the CuBER bar, for $\phi=0$, has to be added to the previous amplitude (see Fig. 13). The new wavelength is obtained subtracting four times the horizontal displacement $u$ (as only a quarter of the wavelength has been considered when solving the cuber problem). Therefore, the regularized misalignment value becomes:

$$
\begin{aligned}
& { }^{n e w} A=A+\mathrm{SF} \cdot v(\phi=0) \\
& { }^{n e w} \lambda=\lambda-4 \mathrm{SF} \cdot u(\phi=\alpha)
\end{aligned}
$$

\subsection{Matrix Homogenized Constitutive Equation}

When the composite is compressed, the structural displacement of fibers generates stresses in the matrix that surrounds the fiber. This effect can be seen in Fig. 17, which shows the transversal stresses (respect fiber direction) and the shear stresses in matrix material, when a finite element micromodel of a RVE is compressed.

These structural stresses, that do not exist in the case of aligned fibers, lead to a matrix failure for lower compression loads. The stress state for which the matrix failure will occur is defined by the constitutive equation of matrix material. In order to obtain the new matrix homogenized constitutive equation, the structural stresses produced by the interaction of matrix with fiber, must be included in the damage constitutive law. Two different structural stresses must be considered: transversal and shear stresses.

As happened when obtaining the homogenized constitutive performance of fiber material, the structural results (loads and displacements) provided by the CuBER problem must be transformed into parameters that can be understood by a constitutive equation, this is: stresses and strains. 


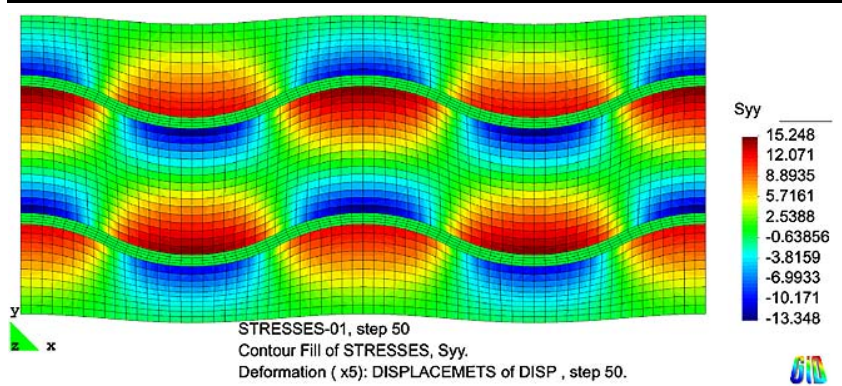

(a) Transversal stresses

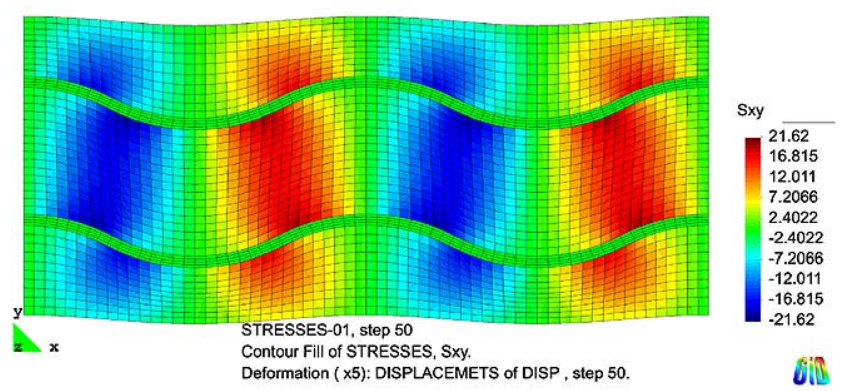

(b) Shear stresses

Fig. 17 Matrix structural stresses produced by fiber displacements

Gonzalez and Llorca [19] pointed out that the fracture in reinforced composites usually occurs due to stress concentrations or localized failures. Following this approach, the stress results from the CuBER problem required to modify the matrix constitutive law are the maximum values of shear and transversal stress provided by the model.

\subsubsection{Matrix Structural Transversal Stress}

Matrix structural transversal stresses are obtained from the elastic restriction applied by matrix over fiber when solving the CuBER problem:

$q=\mathrm{k} \omega$

being $q$ the radial load produced by the elastic restriction over the fiber, $\mathrm{k}$ the matrix Young Modulus and $\omega$ the fiber radial displacement. This last parameter can be computed, according to the equations presented when solving the $\mathrm{Cu}$ BER problem, as:

$$
\begin{aligned}
\omega= & C_{0}+C_{1} \cosh \left(\eta_{1} \phi\right) \cos \left(\eta_{2} \phi\right) \\
& +C_{4} \sinh \left(\eta_{1} \phi\right) \sin \left(\eta_{2} \phi\right)
\end{aligned}
$$

To convert the structural problem into a mechanical one, the radial load $q$ has to be transformed into a stress. This is done dividing it by the affected length. This length is considered equal to the fiber diameter. The resultant stress must be also divided by two, as half of it corresponds to the compression stress on the matrix found above the fiber and the other half corresponds to the tensile stress in the matrix below the fiber (see Fig. 17). The matrix transversal stresses can be obtained with the following expression:

$\sigma_{y y}=\frac{\mathrm{k} \omega}{2 \emptyset_{f}}$

Where $\varnothing_{f}$ is the fiber diameter. The transversal stress has associated a perpendicular one, along fiber longitudinal direction, due to Poisson effects:

$\sigma_{x x}=-v \sigma_{y y}=-v \frac{\mathrm{k} \omega}{\emptyset_{f}}$

According to Fig. 17, the maximum transversal stress in matrix material due to fiber misalignments is found where the misalignment amplitude is maximum. In these places, the fiber displacement corresponds to $\omega(\phi=0)$ (Fig. 13) and its value can be obtained from (60) when solving the $\mathrm{Cu}$ BER problem. The final matrix structural transversal stress state ${ }^{m s} \sigma$ found, in $\phi=0$, is defined by:

$$
\left\{\begin{array}{l}
{ }^{m s} \sigma_{x x}=-v \frac{\mathrm{k} \omega(\phi=0)}{2 \emptyset_{f}} \\
{ }^{m s} \sigma_{y y}=\frac{\mathrm{k} \omega(\phi=0)}{2 \emptyset_{f}}
\end{array}\right.
$$

\subsubsection{Matrix Structural Shear Stress}

The unidimensional model developed to simulate the fibermatrix interaction of the RVE considered cannot obtain directly the matrix structural shear stress, as there are not tangential springs in the model for simulating the matrix shear behavior. Therefore, matrix shear stress is computed using the matrix deformation. Shear stresses are produced by the distortion of an infinitesimal element due to a stress state. This distortion is defined as [64]:

$\gamma=\frac{d u_{x}}{d y}+\frac{d u_{y}}{d x}$

Where the values of $u_{x}$ and $u_{y}$, as well as their derivatives, can be obtained from (63). The shear stress is obtained as:

${ }^{m s} \sigma_{x y}={ }^{m} G \gamma$

According to Fig. 17, the maximum value of matrix shear structural stress is found at the inflection point between the convex and concave arches of circumference. This point corresponds, in the CuBER problem (Fig. 13), to an angle $\phi=\alpha$. The maximum expected shear stress is:

$$
{ }^{m s} \sigma_{x y}={ }^{m} G\left(\left.\frac{d u_{x}}{d y}\right|_{\phi=\alpha}+\left.\frac{d u_{y}}{d x}\right|_{\phi=\alpha}\right)
$$




\subsubsection{Addition of Matrix Structural Stresses in Its} Constitutive Equation

The obtained matrix structural stresses cannot be introduced as real stresses in the matrix constitutive equation, because the equilibrium between the internal and external forces will be lost. If the RVE is loaded with a compression force (in the direction of fibers), the only stresses that can appear in matrix material are in that same direction.

To solve this drawback, the transversal and shear structural stresses are defined as fictitious stresses and are included in the matrix constitutive equation as internal variables. These internal variables will lead to a matrix failure for lower compression loads.

Matrix yield function is defined as:

${ }^{m} \mathbf{F}\left({ }^{m} \sigma, q_{i}\right) \leq 0$

where ${ }^{m} \sigma$ is matrix stress tensor and $q_{i}$ matrix internal variables. When introducing matrix structural stresses in the yield function, (89) becomes,

${ }^{m} \mathbf{F}^{*}\left({ }^{m} \sigma, q_{i},{ }^{m s} \sigma\right) \leq 0$

with ${ }^{m s} \sigma$ the matrix structural stresses or fictitious stresses. This new matrix yield function is defined as:

${ }^{m} \mathbf{F}^{*}\left({ }^{m} \sigma, q_{i},{ }^{m s} \sigma\right)={ }^{m} \mathbf{F}\left({ }^{m} \sigma+{ }^{m s} \sigma, q_{i}\right)$

Figure 17 shows that transversal and shear fictitious stresses are found in different points of the RVE geometry. Hence, the value of matrix fictitious stresses to be included as internal variables are, in a $2 \mathrm{D}$ case, the most critical of the following two:

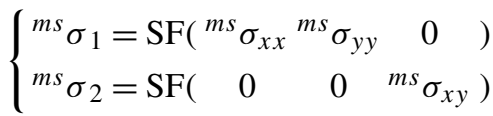

being FS the scale factor defined in (81). This scale factor is necessary to adjust the values obtained when solving the CuBER problem to the real strain that is applied to the RVE due to fiber misalignments.

The two cases to be considered in a two dimensional simulation must be increased to five in the case of a three dimensional simulation. This is because fiber initial misalignments can be found either in the plane $x y$ or in the plane $x z$ (unless it is known and defined the plane in which they are found). Thus, as the fictitious stresses interact with the stresses found in matrix material due to the loading process, it is not possible to know a priori which configuration will damage more the matrix and all of them must be taken into account. Considering that the matrix stress tensor is defined by the following components:

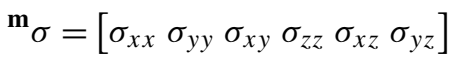

The fictitious stresses that have to be considered in the new constitutive equation are:

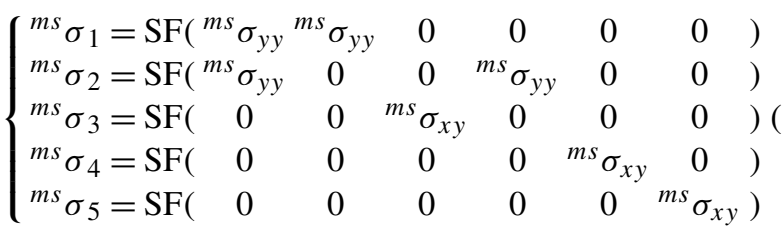

\subsubsection{Matrix Fracture Length}

When solving the damage constitutive equation, using matrix fictitious stresses, an important parameter to be changed in the damage formulation is the fracture length of the finite element [63]. The fracture length defines the amount of energy that can dissipate a finite element of a certain size. In the case of the formulation developed, the fracture length do not depend on the dimension of the finite element but on the dimensions of fiber misalignments. When homogenizing a structural behaviour, each gauss point contains all information of the RVE, independently of the dimension of the gauss point. Thus, even the dimension of the finite element is of, i.e. $5.0 \mu \mathrm{m}$, if the wavelength of the misalignment is of $400.0 \mu \mathrm{m}$, the fracture energy released in the micro-model will correspond to damaging all the wavelength of the fiber misalignment.

The model developed in the CuBER problem represents half the wavelength of fiber misalignments. Therefore, the fracture length that has to be considered when adding the structural fictitious stresses to the constitutive equation is:

$l_{f}=2 R \alpha$

\section{Validation of the Homogenization Method}

In order to validate the approach used to include the effects of fiber misalignment in fiber and matrix constitutive equations, the results of a finite element micro-model of the representative volume element (RVE) simulated are compared with the results obtained with the formulation proposed. It will be shown that the behavior of the RVE is the same in both models.

\subsection{Models Description}

A basic composite configuration has been defined to verify the agreement between the finite element micro-model and the new formulation proposed. The composite defined is made of glass-fibers embedded in an epoxi matrix. The values considered for each component material are average values, obtained from literature. These are shown in Table 1.

Matrix material has been described with the modified damage constitutive law defined in previous section. The 
Table 1 Composite components mechanical properties used to validate the homogenized constitutive equations

\begin{tabular}{lc}
\hline Matrix Properties & \\
Elastic Modulus & $3.00 \mathrm{GPa}$ \\
Poisson Modulus & 0.30 \\
Tensile Strength & $90.00 \mathrm{MPa}$ \\
Fracture Energy & $1.00 \mathrm{~kJ} / \mathrm{m}^{2}$ \\
Fiber Properties & \\
Elastic Modulus & $80.00 \mathrm{GPa}$ \\
Poisson Modulus & 0.00
\end{tabular}

yield function used to obtain the stress state in which damage starts in matrix material is based in the norm of the principal stresses $[58,63]$, which non-homogenized expression is:

$\mathbf{F}\left(\sigma, q_{i}\right)=\sqrt{\sigma_{1}^{2}+\sigma_{2}^{2}+\sigma_{3}^{2}}-\sigma^{\max } \leq 0$

Being $\sigma_{i}$ the principal stresses values obtained from the matrix stress tensor and $\sigma^{\max }$ the matrix tensile strength.

Using the fact that fiber buckling occurs before fibers reach their limit elastic strength, fibers are considered to be an elastic material. The other parameter required to define fiber elastic properties is the Poisson modulus, which is considered equal to zero. This is done because fiber transversal deformations, due to their longitudinal strains, are considered negligible.

The misalignment properties considered for the basic model are the ones defined by Jochum and Grandidier [15]. These authors obtain fiber misalignment properties using an optical microscope. The results obtained from these observations are:

1. The diameter of the fiber is of $7.0 \mu \mathrm{m}$

2. Amplitude is one to twice the fiber diameter

3. Wave length is comprehended between 150 and $300 \mu \mathrm{m}$

With these values in mind, the misalignment configuration defined for the basic model of the RVE are the ones shown in Fig. 18 and described in Table 2. The volumetric participation of fiber and matrix in the composite is obtained from the geometry shown in Fig. 18. This figure shows that only two fibers are considered in the RVE model. This small fiber proportion has been chosen in order to see more clearly the interaction between fiber and its surrounding matrix.

To be certain of the good performance of the formulation proposed, six different variations of the basic RVE model have been defined. Each one modifying the different parameters that affect the micro-mechanical behavior of the RVE:

Bmod: Basic model with the properties described in Table 2 and Fig. 18.
Table 2 Misalignment properties and volumetric participations used to validate the homogenized constitutive equations

\begin{tabular}{lr}
\hline Fiber diameter & $7.0 \mu \mathrm{m}$ \\
Amplitude & $10.5 \mu \mathrm{m}$ \\
Wave length & $225.0 \mu \mathrm{m}$ \\
Fiber Vol. particip. & $7.0 \mu \mathrm{m}$ \\
Matrix Vol. particip. & $93.0 \mu \mathrm{m}$ \\
\hline
\end{tabular}

Table 3 Parameters defining the different RVE models developed

\begin{tabular}{lcclcc}
\hline RVE & $\begin{array}{l}\emptyset_{f} \\
{[\mu \mathrm{m}]}\end{array}$ & $\begin{array}{l}\mathrm{A} \\
{[\mu \mathrm{m}]}\end{array}$ & $\begin{array}{l}\lambda \\
{[\mu \mathrm{m}]}\end{array}$ & $\begin{array}{l}{ }^{f} E \\
{[\mathrm{GPa}]}\end{array}$ & $\begin{array}{l}{ }^{m} E \\
{[\mathrm{GPa}]}\end{array}$ \\
\hline Bmod & 7.0 & 10.5 & 225.0 & 80.0 & 3.0 \\
DfiM & 5.0 & 10.5 & 225.0 & 80.0 & 3.0 \\
DfiL & 9.0 & 10.5 & 225.0 & 80.0 & 3.0 \\
AmpM & 7.0 & 7.0 & 225.0 & 80.0 & 3.0 \\
AmpL & 7.0 & 14.0 & 225.0 & 80.0 & 3.0 \\
FreM & 7.0 & 10.5 & 150.0 & 80.0 & 3.0 \\
FreL & 7.0 & 10.5 & 300.0 & 80.0 & 3.0 \\
Wmat & 7.0 & 10.5 & 225.0 & 80.0 & 1.5 \\
Sfib & 7.0 & 10.5 & 225.0 & 230.0 & 3.0
\end{tabular}

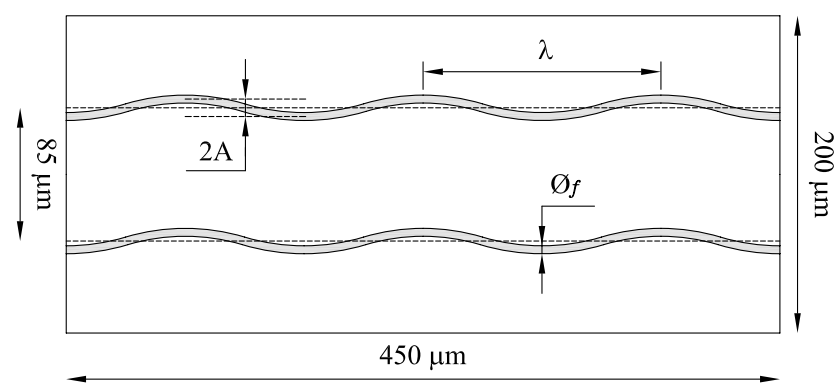

Fig. 18 Geometry of the Representative Volume Element simulated

DfibM \& DfibL: Models with the same properties as Bmod but in which the fiber diameter has been reduced and increased, respectively.

AmpM \& AmpL: Models that vary the initial misalignment amplitude, reducing and increasing it respectively.

FreM \& FreL: These two models modify the initial misalignment of Bmod model by reducing and increasing the fiber wavelength $(\lambda)$.

Wmat \& Sfib: Last variation of Bmod model consist in modifying the stiffness of the component materials, reducing matrix stiffness and increasing fiber stiffness, respectively.

The values of the different parameters applied to the geometry, as well as the name assigned to each model, are described in Table 3.

All different RVE configurations described in Table 3 have been modeled with a finite element micro-model and 


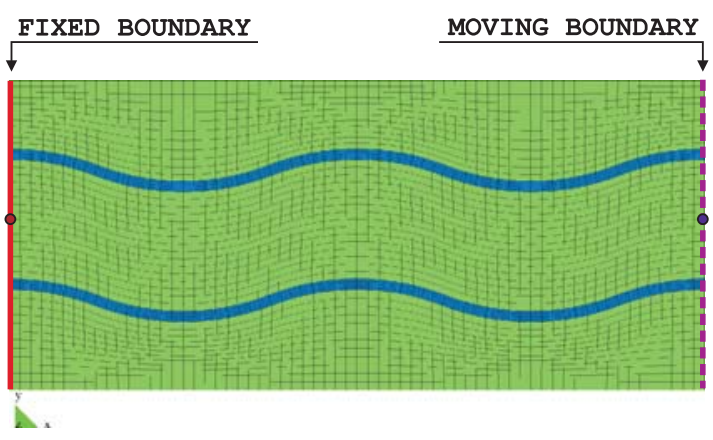

Fig. 19 micro-Bmod finite element model. Mesh and boundary conditions defined

with the new formulation proposed. Each micro-model is labeled with the word micro, thus, the micro-model of Bmod RVE is named micro-Bmod. The models developed with the new formulation are labeled with the word RoM which names, the Bmod RVE, as RoM-Bmod.

\subsubsection{Finite Element micro Models}

The finite element micro-models have been defined using a two dimensional plane stress formulation. The geometry has been modeled using linear quadrilateral elements, which number varies between 2880 elements in the FreM and FreL models to 3185 elements in all other models. All models have 48 elements in direction $y$ (each fiber is modeled with four elements along its thickness to capture with more accuracy its bending stiffness) and 60 or 64 elements, depending on the RVE cell simulated, in direction $x$. The mesh used for the micro-Bmod model is displayed in Fig. 19.

The difference between the mesh shown in Fig. 19 and the meshes developed for all other RVE models is found in the fiber geometry. Which is modified in order to follow the different fiber misalignments defined.

Figure 19 also shows the boundary conditions defined in all micro-models. The longitudinal movement in left boundary is fixed to zero while the longitudinal movement in the right one, displayed with a dashed line, is moving towards the fixed boundary. This movement generates a compression on the RVE. The two points plotted in both boundaries correspond to the points in which the transversal displacement has been fixed to zero, in order to stabilize the model and avoid undesired translations of it.

Two different materials have been defined in each micromodel. Matrix material is simulated with a damage constitutive law while fiber material is characterized with an elastic law. The mechanical properties of both materials have been already defined in Table 1 .

\subsubsection{Finite Element RoM Models}

The RoM models obtain the performance of the different RVE defined using the homogenized constitutive equations

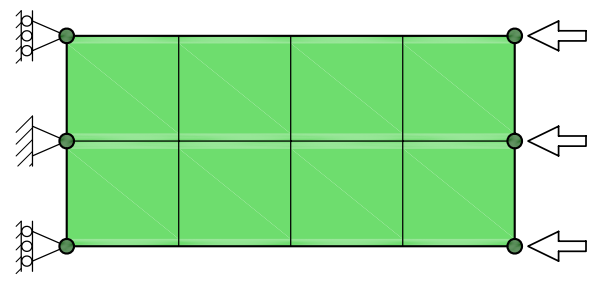

Fig. 20 RoM finite element models. Mesh and boundary conditions defined

for matrix and fiber presented in Sect. 4. The finite element models developed also use a plain stress formulation. The mesh used for these simulations contains eight linear quadrilateral finite elements. This mesh is shown in Fig. 20.

The arrows shown in the right side nodes define the displacement direction applied to those nodes. This displacement is the same that has been applied to the RVE micro models.

The RoM models are defined with a single composite material, which constitutive performance is obtained using the serial/parallel mixing theory. Fiber and matrix properties are the same defined for the micro model. The volumetric participation considered for each component material has been obtained from Fig. 18.

It is important to remark the difference in the number of mesh elements required by the micro models and the RoM models. The number of elements required by the micro models is three orders of magnitude larger than the number of elements required by the RoM models. Therefore, if the results provided by both procedures are similar, the new formulation proposed will offer the possibility to do large scale simulations, taking into account the fiber-matrix micro-structural interaction due to fiber initial misalignments. This possibility is unbearable by nowadays personal computers, if a complete homogenization method wants to be used.

\subsection{Results to be Studied in the Code Validation}

The study of the results obtained with the different models developed to validate the proposed formulation is done comparing the global performance of the micro models with the RoM models. The comparison of the specific performance of each model is not possible because the results obtained are completely different in both simulations. However, as the interest lays on the global performance of the RVE cell, if the global results are similar, the formulation can be considered to be valid.

The global performance expected from the RVE when including fiber misalignments is a reduction on the composite stiffness and an initial failure on the composite for lower loads, due to the failure of matrix material. Therefore, these two effects are the ones to be compared. The reduction of 


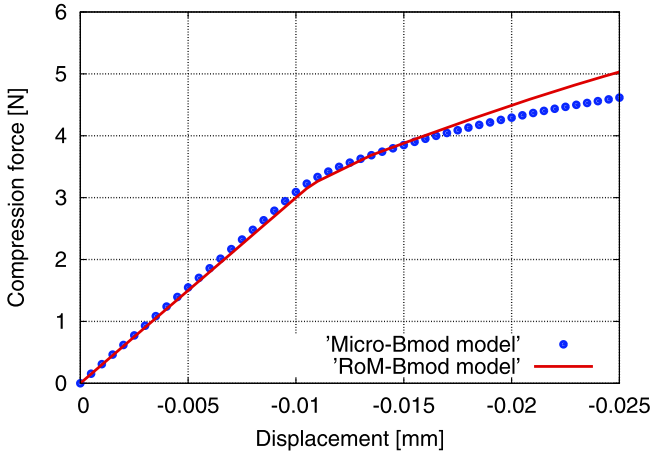

Fig. 21 Force-displacement graph showing the global behavior of the Micro-Bmod and RoM-Bmod models

composite stiffness is produced by the reduction of fiber stiffness due to its micro-structural displacements; and the failure of matrix for lower loads is produced because matrix stresses are increased with the shear and transversal structural stresses, result of the fiber structural displacements.

The validation of the formulation proposed is made comparing the structural results of both RVE models, micro and RoM, studying the load at which the composite failure starts and the stiffness of the composite before and after failure. This comparison is made using the force-displacement curves obtained for the RVE. A detailed study is performed, first, for the Bmod model. Afterwards, all other RVE cells simulated are compared.

\subsubsection{Comparison Between the Bmod Model and the micro Model}

The global performance of the RVE in the Bmod model is defined by two main parameters: the load for which damage appears in matrix and the stiffness of the composite, before and after, matrix failure. These two parameters can be studied with the force-displacement graph obtained for each simulation of the RVE. Both graphs are represented in Fig. 21.

Figure 21 shows that the global behaviour of both models is the same. Both force-displacement graphs are close enough to consider that the RoM model performance is practically the same shown by the micro model and, therefore, correct.

A better understanding of the global performance shown by the RoM-Bmod model can be obtained form the stressstrain graphs, of the composite and of its constituent materials. The stresses and strains represented correspond to the longitudinal direction of the RVE. These graphs, shown in Fig. 22, are displayed together with the results obtained from the same RVE simulation when the fiber misalignment phenomenon is not considered.

Figure 22a shows the effect over the composite of the formulation developed. Damage in the composite starts for

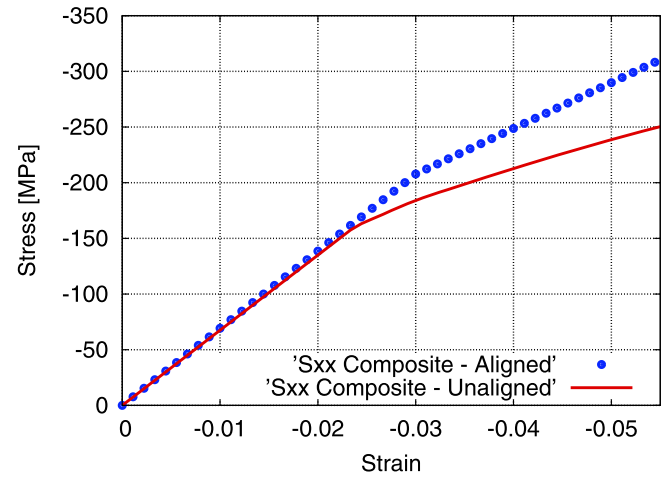

(a) Composite

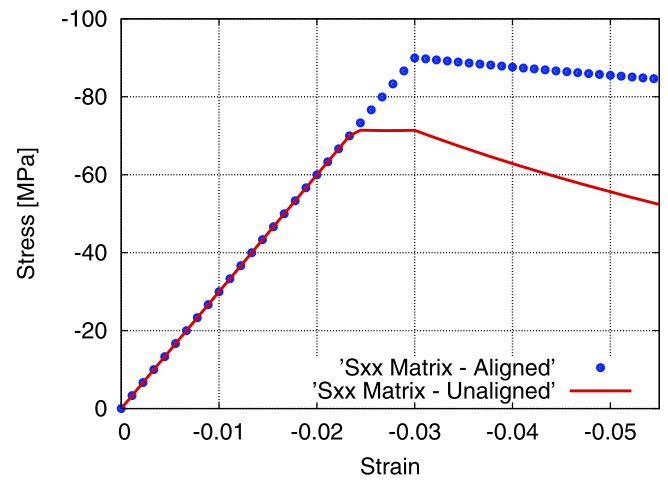

(b) Matrix

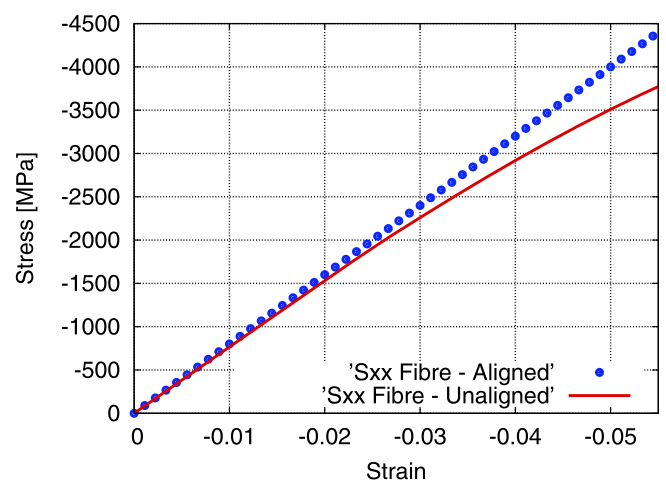

(c) Fiber

Fig. 22 Stress-strain graphs obtained for the RVE with the serial/parallel mixing theory, with aligned and misaligned fibers

lower loads and, once damage has started, the stiffness on the composite is reduced.

Damage in the composite starts before because matrix damage appears for a longitudinal stress more than a $20 \%$ lower than in the case in which fiber misalignments are not considered (Fig. 22b). Fibers in the misaligned model show a small reduction of their elastic stiffness from the beginning of the stress-strain graph. This reduction becomes more important, and keeps increasing, when damage appears in matrix (Fig. 22c).

How fiber initial misalignments contribute to fiber stiffness reduction can be seen studying the fiber structural dam- 


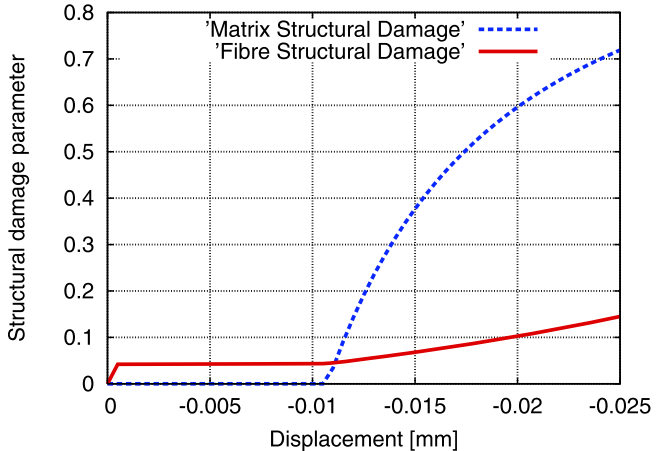

Fig. 23 Fiber and matrix structural damage. RoM-Bmod model

age parameter. This is represented in Fig. 23, together with the matrix damage parameter. This figure shows an initial damage in fiber material due to its initial misalignment. This damage value remains constant until matrix degradation starts. At this point, fiber structural damage starts increasing exponentially as matrix cannot restrain the fiber movement anymore.

Another important information obtained from the results of the RoM model is that, of the two different fictitious stress tensors added to the matrix homogenized constitutive law (92), damage is produced by the stress tensor containing the fictitious structural shear stress. This is a relevant issue, as it shows that the proposed formulation agrees with the different formulations existing in literature, in which fiber buckling has a strong dependence on the shear strength of matrix material.

\subsubsection{Comparison of the Results Obtained with the micro and RoM Models for all RVE's Considered}

Here is validated the global behavior of the RVE when the different parameters included in the CuBER problem are modified. These parameters are fiber initial misalignments (amplitude and wavelength), fiber diameter and fiber and matrix stiffness. A detailed description of the different RVE models developed has been already shown in Table 3 .

As has been done with the Bmod simulation, the global behavior of the composite is studied with the force-displacement graph obtained for each RVE simulation. Four different graphs are shown, each one containing the simulations in which one of the parameters defining the CuBER performance is modified. All graphs contain also the result obtained with the Bmod models, which stands as reference for the two other results displayed.

Figure 24 includes the global performance of the models in which the amplitude is increased or reduced, respect the value defined for the Bmod model. Figure 25 shows the results for the models in which the wavelength is modified. Figure 26 shows the results for the models with different fiber diameters. Finally, Fig. 27 shows the results for the

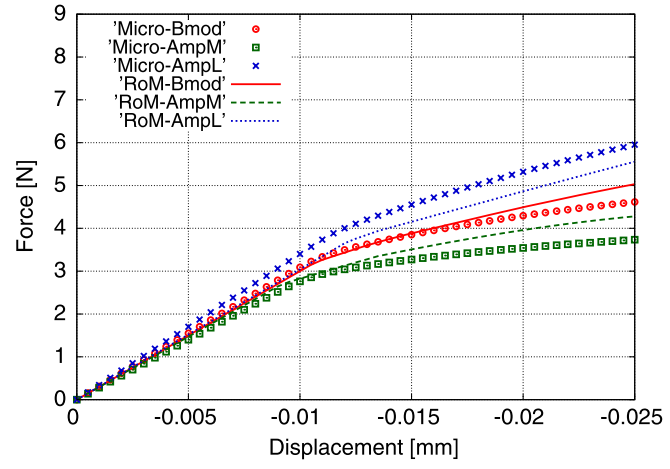

Fig. 24 Force-displacement graph showing the global behavior of the models in which the amplitude is modified

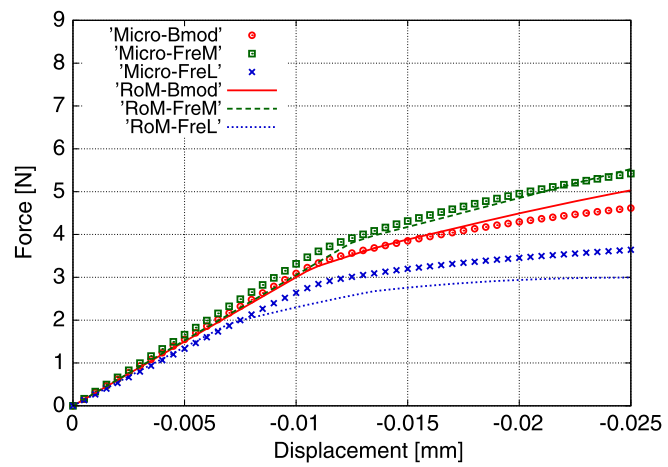

Fig. 25 Force-displacement graph showing the global behavior of the models in which the wavelength is modified

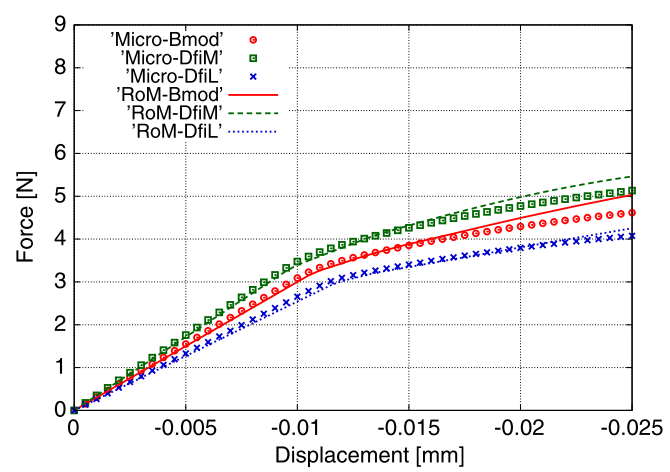

Fig. 26 Force-displacement graph showing the global behavior of the models in which the fiber diameter is modified

models in which the stiffness of matrix and fiber are modified.

As can be seen in Figs. 24 to 27, the global behavior of the RVE obtained with the new proposed formulation and the global behavior obtained from the micro models is nearly the same in all cases. The modification of one of the parameters defining the CuBER problem modifies the loaddisplacement point in which damage appears in the composite, as well as the composite stiffness before and after 


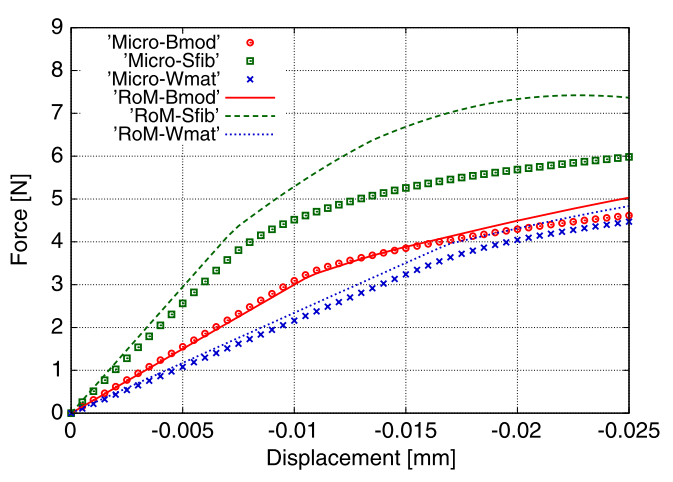

Fig. 27 Force-displacement graph showing the global behavior of the models in which the stiffness of the composite constituents is modified

damage. The new mechanical performance obtained is the same with the RoM and the micro models.

Figure 24 shows that the initial elastic stiffness of the composite suffers small variations when the misalignment amplitude is modified. The differences between the load at which degradation starts in the composite is nearly unnoticeably when comparing the global behavior of the micro and the RoM models. The error obtained in the composite stiffness after the apparition of damage seems to increase if fiber amplitude is increased. However, the error is small enough to consider the results correct.

The comparison of the RVE performance when the misalignment wavelength is increased or reduced (Fig. 25) shows that the prediction of the two stiffnesses shown by the composite, before and after damage, are very similar between the micro and the RoM models. However, a larger difference is found in the load for which damage appears, specially when the wavelength is reduced.

The comparison of the results obtained for the models in which fiber diameter is modified (Fig. 26) shows a perfect agreement between the micro and the RoM model. This agreement is found in all the composite stiffnesses and in the load for which the composite degradation starts.

The last parameters modified are the fiber and matrix stiffness, which results are shown in Fig. 27. This figure shows that, while the prediction of the RVE performance with the RoM model is nearly the same that is obtained with the micro model when matrix stiffness is reduced, the prediction in the case in which fiber stiffness is increased is not as accurate. The difference between both models is most significant when comparing the stiffness obtained after the apparition of damage in the composite. So, composites with larger fiber stiffness will require a better calibration of the model in order to adjust the micro-structural performance of both models.

With all results shown, it can be concluded that the developed formulation is capable of including the microstructural effects of fiber misalignment in the serial/parallel mixing theory. This is done defining homogenized constitutive equations for fiber and matrix, which are obtained based on the solution of the CuBER problem. The formulation developed provides accurate results when it is compared with the results obtained from a finite element micro model. It is able to capture modifications in fiber misalignments and fiber and matrix properties; being those the parameters defined in literature related to the fiber buckling problem. Also, the failure cause of matrix material has been found to be due to shear stresses in all simulations performed.

\section{Numerical Procedure to Obtain the Compression Strength of Composites}

\subsection{General Description of the Numerical Procedure Developed}

Section 4 has described a new methodology and formulation to include into the serial/parallel mixing theory the effect of the micro-structural interaction between fiber and matrix, due to fiber initial misalignments. To do so, the effects of this micro-structural interaction have been included in the constitutive equations of fiber and matrix, defining an homogenized constitutive law for each material. The good performance of this new methodology has been proved in the numerical validation shown in Sect. 5 .

This section shows that if the new formulation developed is properly included in a finite element code, the compression strength of the composite is obtained straightforward from the constitutive performance of the composite constituents [65].

In order to obtain a general procedure to calculate the compression strength of composite materials, there are other cases that have to be considered, as they are not included in the homogenized constitutive equations for fiber and matrix obtained in Sect. 4. These cases are the situation in which fibers do not have initial misalignments or when fiber initial misalignments are too small to affect significantly the constitutive behavior of fiber and matrix. A general procedure to obtain the compression strength of composites has also to be able to deal with unloading processes and must provide a different composite performance depending on the sign of the load applied to it: tensile load or compression load.

The procedure proposed to obtain the compression strength of composites, is based in a phenomenological representation of the micro-mechanical performance of the composite constituents. This procedure, besides including fiber initial misalignments in the constitutive equations of fiber and matrix, also considers other fiber configurations and it takes into account the loading history of the composite. 


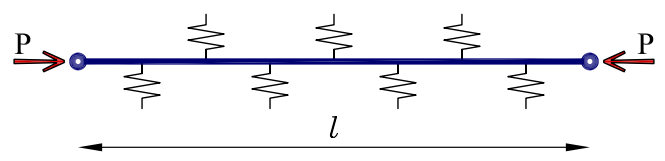

Fig. 28 Geometry considered to obtain the buckling load of straight fibers

\subsection{Compression Strength in Fibers Without Initial Misalignments}

There are situations in which fiber initial misalignments are too small to be recorded by the testing methods available, or in which the misalignment wavelength is too large to affect the fiber performance. To take into account these two particular cases, or any other case in which fiber misalignments can be considered non-existent, it is necessary to obtain a formulation to obtain the maximum compression force that can be applied to the composite due to fiber buckling effects.

As has been done with misaligned fibers (Sect. 4), this problem can be solved using the existing analogy between the fiber-matrix system and the case of a bar in an elastic foundation. Thus, the fiber buckling load can be computed considering fibers a bar and the matrix in which they are embedded the elastic foundation. Matrix stiffness provides the elastic variable of the foundation. If damage appears in matrix material, the elastic variable defining the foundation will be reduced proportionally to the degradation found in matrix.

The procedure developed to obtain the compression strength of composite materials not only has to find the maximum load that can be applied to the composite but it also has to provide its post critical strength. Therefore, once fibers without misalignment have buckled, it is necessary to find their new configuration and constitutive performance.

Once the fiber has buckled it will adopt a new curved geometry, similar to the one found in the case of fibers with initial misalignments, that can be defined by a wavelength and an amplitude. So, after fiber buckling, the new fiber geometry is computed and the composite post-critical behavior is obtained using the formulation already developed for fibers with an initial misalignment.

\subsubsection{Buckling Stress for Fibers Without Initial Misalignments}

In the case of fibers without initial misalignment, the buckling stress is obtained from the critical buckling load of a straight bar in an elastic foundation. The geometry of the problem is shown in Fig. 28.

To obtain the critical buckling load of a bar under unilateral elastic restriction the energy method described by Timoshenko [66] is used. This is an approximate method in which the strain energy of bending $(\Delta U)$ is compared with the decrease in the potential work of the load $(\Delta T)$. A lower value of $\Delta T$ than $\Delta U$ means that the deflection of the bar requires an increase of the potential energy of the system; or, which is the same, means that the system is stable. On the other hand, if $\Delta U<\Delta T$, the deflection of the bar is accompanied by a decrease in the potential energy of the system, which makes it unstable. The load that separates both situations is defined as the critical buckling load. This procedure is fully described in Martinez [67] and the final expression of the buckling load is:

$P_{c r}=\frac{\pi^{2} E I}{l^{2}}\left(n^{2}+\frac{\mathrm{k} l^{4}}{n^{2} \pi^{4} E I}\right)$

where the $n$ value defines the buckling mode of the bar. According to (97) the value of $n$ that makes $P_{c r}$ minimum depends on the elastic modulus of the foundation and the length and stiffness of the bar. So, the buckling mode of the bar will depend on the value of these parameters.

One of the main problems found with the formulation described to obtain the buckling load of a bar in an elastic foundation is the definition of the buckling length $l$ to be used. In the case considered, fiber vertical movement is restrained with the same intensity along the whole fiber length.

In order to consider the most unfavorable situation, the buckling length considered corresponds to the buckling length that minimizes the critical buckling load. This buckling length is obtained searching the value of $l$ that minimizes the function $P_{c r}(l)$ defined in (97):

$\frac{d P_{c r}}{d l}=0.0$

Expression (98) defines a critical buckling length, which value is:

$l_{c r}=n \pi \sqrt[4]{\frac{E I}{\mathrm{k}}}$

If the buckling length defined in (99) is replaced in (97), the critical buckling load obtained is:

$P_{c r}=2 \pi \sqrt[2]{\mathrm{kEI}}$

This last expression shows that the dependence on the buckling mode disappears from the critical load value, which provides a unique buckling load value for all buckling modes.

Once knowing the critical buckling load at which fibers buckle, this load has to be converted to a critical stress in order to be included into the constitutive equation of fiber material. This conversion is made dividing the critical load by fiber area:

$\sigma_{c r}=\frac{2 \pi \sqrt[2]{\mathrm{kEI}}}{\pi \varnothing_{f}^{2} / 4}=\frac{8 \sqrt[2]{\mathrm{k} E I}}{\emptyset_{f}^{2}}$ 


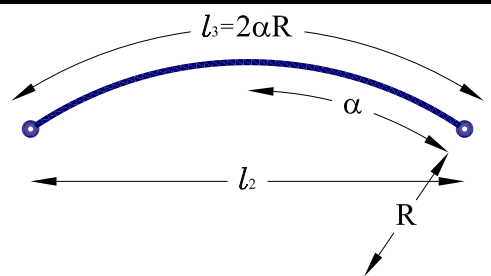

Fig. 29 Fiber geometry just after reaching the critical buckling load

The critical stress defined in (101) corresponds to the critical stress in fiber longitudinal direction. Therefore, it has to be compared with the parallel component of the stress tensor of fiber material, obtained with the serial/parallel mixing theory.

\subsubsection{Geometry of Fibers After Buckling}

When fibres buckle, they become misaligned and their mechanical performance can be obtained with the misaligned fiber formulation developed. The new fiber geometry is obtained using the following hypothesis:

i. Fiber new geometry is considered to be contained in an arch of circumference. This approximation has been already used when solving the CuBER problem and it has been already proved its validity. The new fiber geometry is be defined by a radii $R$ and an arch angle $\alpha$.

ii. When fibers buckle, they release part of their axial deformation. This implies an increase of the fiber length and a reduction of their longitudinal stress.

iii. New fiber geometry is determined by the equilibrium between matrix efforts over fibers and fiber normal stresses.

Based on these hypothesis, the final geometric configuration of fibers after buckling is shown in Fig. 29. Where,

$l_{2}=l_{c r}\left(1-\varepsilon_{c r}\right) ; \quad \varepsilon_{c r}=\frac{\sigma_{c r}}{E}$

and

$\sin (\alpha)=\frac{l_{2}}{2 R}=\frac{l_{c r}\left(1-\varepsilon_{c r}\right)}{2 R}$

being $l_{c r}$ the bar length before applying the compression force and $l_{2}$ the bar length just before buckling.

The new geometry displayed in Fig. 29 leads to a new axial force over fiber material result of the new fiber length. This force can be obtained as:

$P=E A_{f} \frac{l_{c r}-l_{3}}{l_{c r}}=E A_{f} \frac{l_{c r}-2 \alpha R}{l_{c r}}$

This axial force, $P$, has to be in equilibrium with the external load applied to the fiber, result of the matrix elastic restrain. This equilibrium is shown in Fig. 30.

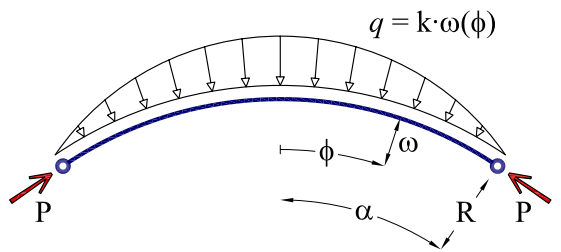

Fig. 30 Equilibrium defined between compression force in fiber and elastic restrain of matrix

Here, the expression of the radial displacement of the fiber is obtained from the circumferential properties of the new geometry:

$\omega(\phi)=R\left(1-\frac{\cos \phi}{\cos \alpha}\right)$

The equilibrium shown in Fig. 30 leads to:

$P=\int_{0}^{\alpha} \mathrm{k} \omega(\phi) R \mathrm{~d} \phi$

And the new fiber geometry, defined by the arch radius $R$ and the internal angle $\alpha$ can be obtained with the non-linear system defined by (103) and (106). After solving the integral defined in (106) and rearranging both equations, the system to be solved becomes:

$$
\left\{\begin{array}{l}
\frac{l_{c r}\left(1-\varepsilon_{c r}\right)}{2 \sin \alpha}-R=0 \\
\mathrm{k} R^{2}\left(\alpha-\cos \alpha \ln \left[\tan \left(\frac{\pi}{4}+\frac{\alpha}{2}\right)\right]\right) \\
\quad-E A_{f} \frac{l_{c r}-2 \alpha R}{l_{c r}}=0
\end{array}\right.
$$

This non-linear system of equations is solved using a Newton Raphson method [68].

Once having the new geometry radius and internal angle, both values have to be converted to the amplitude, $A$, and wave-length, $\lambda$ required by the CuBER formulation (108).

$$
\begin{aligned}
& A=R(1-\cos \alpha) \\
& \lambda=4 R \sin \alpha
\end{aligned}
$$

With these new misalignment parameters, the postcritical behavior of fibers is obtained using the formulation of fibers with initial misalignments.

\subsection{Compression Strength in Fibers with Initial Misalignments}

When fibers have initial misalignments, their compression strength is obtained using the homogenized constitutive equations presented in Sect. 4. With this formulation, degradation in matrix appears for lower compression stresses and fiber stiffness is reduced. This reduction of fiber stiffness becomes more significant as matrix damage increases because 


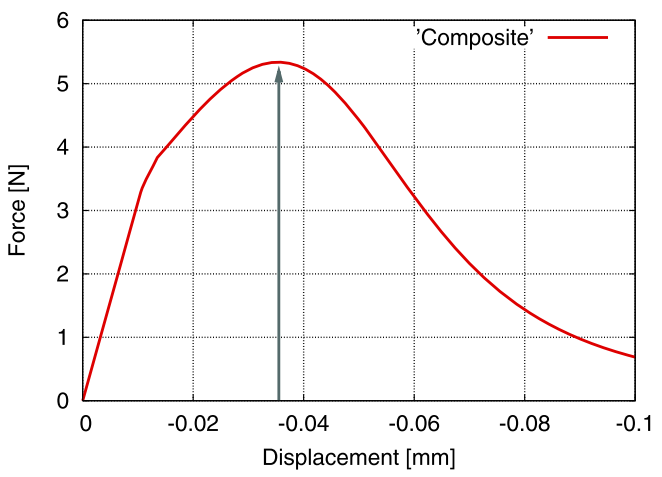

(a) Force-displacement graph

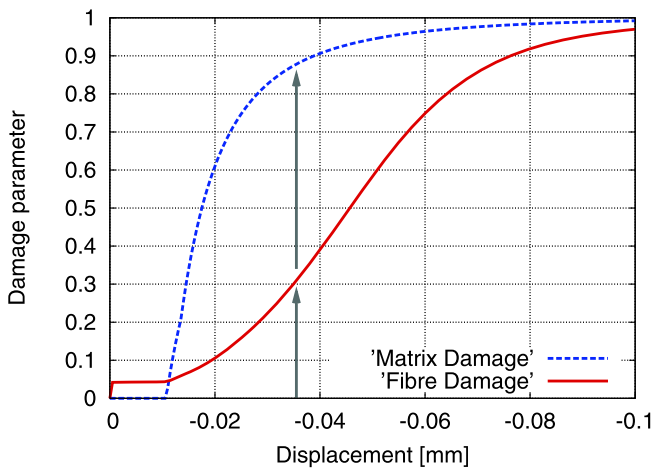

(b) Damage in fiber and matrix

Fig. 31 Post critical performance of a composite with fibers with initial misalignments

the confinement effect provided by matrix is weaker. As the load in the composite increases, it is possible that the damage in matrix and the structural damage in fiber makes the composite unable to support the applied load. At this point the maximum compression force that can be applied to the composite is reached, and the stress-strain relation begins to decrease providing the post-critical path of the composite.

This is shown in Fig. 31, in a force-displacement graph of the composite and in a damage evolution graph for matrix and fiber. These graphs show the displacement in which the maximum load is reached (disp $=-35.5 \mu \mathrm{m}$ ). The composite properties defined to obtain this graph correspond to the properties of the RoM-Bmod model defined in Sect. 5.

Figure 31 shows that the formulation developed to take into account fiber initial misalignments and the interaction between fiber and matrix provides the buckling strength of the composite without requiring any additional formulation. When matrix cannot restrain fibers any more, their initial misalignment starts increasing indefinitely, being unable to provide more strength to the composite. This situation has been already defined as fiber buckling by Akbarov and Kosker [13].

Eventually, damage in matrix can reach a level in which the confinement effect becomes non-existent. When this situation is reached, the equations that provide the structural

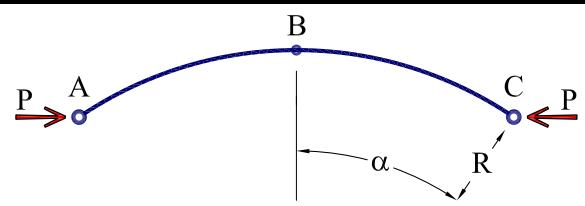

Fig. 32 Geometry of the curved bar problem when matrix is completely damaged

displacement of the fiber are not longer valid and must be replaced by the equations of a curved bar.

Another situation that must be considered is the case in which the misalignments are too small (very large wavelength with small amplitudes) to affect significantly the composite performance. In these situations is possible that the fiber buckle like a straight fiber before the homogenization procedure affects the composite performance. Because there is no way to know a priori if the compression strength of composites with misaligned fibers will be determined by the homogenized constitutive equations or if fibers will buckle because misalignments are too small, it is necessary to verify at each load step if the stresses applied over the misaligned fiber are larger than the buckling stresses.

In the case of misaligned fibers, the buckling stress is obtained using the existing analogy between the composite micro-structural configuration and the case of a curved bar in an elastic foundation, for which the buckling load can be obtained.

\subsubsection{Fiber Performance When Matrix is Completely Damaged}

When matrix is completely damaged the equations used to solve obtain the constitutive performance of fiber and matrix due to fiber initial misalignments are no longer valid. Therefore, the CuBER formulation must be replaced by the formulation of a curved bar. The geometric configuration of the problem considered is shown in Fig. 32.

The displacements of the bar shown in Fig. 32, for the applied force $P$ can be obtained easily using the unit force method.

The displacement values obtained for the case of a curved bar, without any kind of restriction, can be used as were used the displacements obtained when solving the CuBER problem to obtain the homogenized constitutive law of fiber material. Therefore, the new structural stiffness for fiber becomes:

${ }^{f s} E_{n m}=\frac{P}{A_{f i b}} \frac{0.25 \lambda}{u_{C}}$

with,

$u_{C}=-\frac{P R^{3}}{E I} \int_{0}^{\alpha}(\cos \phi-\cos \alpha)^{2} \mathrm{~d} \phi$ 


$$
=-\frac{P R^{3}}{E I}\left[\alpha\left(\frac{1}{2}+\cos ^{2} \alpha\right)-\frac{3}{2} \cos \alpha \sin \alpha\right]
$$

This new value of fiber structural stiffness is used to obtain the homogenized constitutive performance of fiber material, if the matrix confinement of fibers becomes negligible. This situation is considered to occur when the new fiber structural stiffness is larger than the structural stiffness obtained with the CuBER problem (79).

\subsubsection{Buckling Stress of Misaligned Fibers}

The critical buckling stress for fibers with initial misalignments is obtained from the energy equilibrium, following the same procedure used to obtain the buckling stress of straight fibers. The final expression of the buckling load is [67]:

$P_{c r}=\frac{\alpha^{2}}{\pi^{2}} \frac{\frac{E I}{R^{2}}\left[1-\left(\frac{\pi n}{\alpha}\right)^{2}\right]^{2}+\mathrm{k} R^{2}}{\left(1+\frac{E I}{\mathrm{k} R^{4}}\right) n^{2}}$

In the case of fibers with initial misalignments, the buckling length considered corresponds to the length between two consecutive inflection points of the misalignment. This is:

$l=\lambda / 2$

In order to obtain the critical buckling load of the system, is required to evaluate expression (111) for different $n$ values until the minimum value of $P_{c r}$ is found. The buckling stress will be calculated as:

$\sigma_{c r}=\frac{P^{*}}{A_{f}} \quad$ with $P^{*}=\min \left\{P_{c r}(n=i) ; i \in \mathbf{N}\right\}$

If at any load step fiber parallel stresses are larger than $\sigma_{c r}$, fiber buckling will occur and the new misalignment configuration must be computed. The new amplitude and wavelength are obtained following the same procedure used for straight fibers. However, in this case, the length used to obtain the new geometry corresponds to the buckling length $l$ (defined in (112)) divided by $n$ (buckling mode).

\subsection{Implementation of the Numerical Procedure Proposed in a Finite Element Code}

The numerical procedures and formulations developed to obtain the compression strength of composite materials due to the fiber buckling phenomenon are included in PLCd [40], a finite element code that deals with composite materials using the serial/parallel mixing theory.

The fiber buckling formulation not only affects the constitutive performance of fiber and matrix materials but also requires to establish communication between both materials, as the degradation and the stress-strain state of each one affects the other one. This situation forces the compression strength procedure to interact in different places of the serial/parallel mixing algorithm to gather all information required by the fiber buckling formulation and to provide a correct constitutive performance of the composite.

This section describes, using flow charts, how the formulation defined in this section and in Sect. 4 is implemented in the serial/parallel mixing algorithm. The first flow chart shown, in Fig. 33, provides a description of the modifications required in the serial/parallel mixing theory to include the fiber buckling procedure. In this flow chart all procedures related to the compression strength formulation are shaded. As can be seen it is necessary to modify the ser$\mathrm{ial} /$ parallel mixing theory algorithm in two different places.

The CuBER problem is solved for fist time just after obtaining the prediction of the strain tensors for fiber and matrix, in order to obtain the fictitious structural stresses required by the matrix homogenized constitutive equation. The CuBER problem is solved only if fibers are misaligned. In case of aligned fibers, there is no need to solve the CuBER problem because matrix structural stresses are zero.

The second time in which the serial/parallel mixing theory algorithm has to be modified is after solving the matrix homogenized constitutive law. At this point, the code enters into the fiber buckling algorithm (or compression strength algorithm), described in Fig. 34, with the matrix damage parameter obtained from matrix constitutive law. This damage parameter is required to obtain the fiber homogenized constitutive performance. The code enters into the fiber buckling algorithm in each iteration performed by the serial/parallel mixing theory, as the matrix damage parameter can be modified in each iteration and these modifications can affect fiber constitutive performance.

The fiber buckling algorithm, described in Fig. 34, calculates the parameters required by fiber and matrix homogenized constitutive equations for all the possible situations considered to obtain the compression strength of composite materials. Therefore, three different numerical procedures can take place depending on the situation in which the fibermatrix system enters the algorithm. This situation is described by three different parameters: fiber radius, misalignment amplitude and the parallel strain of fiber material.

If fiber radius is equal to zero, the code does not take into account the fiber buckling problem. Therefore, no calculations are performed and the code leaves the algorithm. On the other hand, if fiber radius is non-zero, two different situations can be found: that fibers are aligned or misaligned. The misalignment amplitude is zero if the fibers are aligned.

In the case of aligned fibers, the code checks if they are in tension or in compression $\left({ }^{f} \varepsilon^{*}>0\right.$ or ${ }^{f} \varepsilon^{*}<0$, respectively). If fibers are in tension the code leaves the algorithm. If they are in compression, the parallel component of the stress tensor of fibers is compared with the critical buckling 
Fig. 33 Flow chart with the implementation of the compression strength algorithm in the serial/parallel mixing theory. The algorithm used to solve the fiber buckling problem is described in Fig. 34

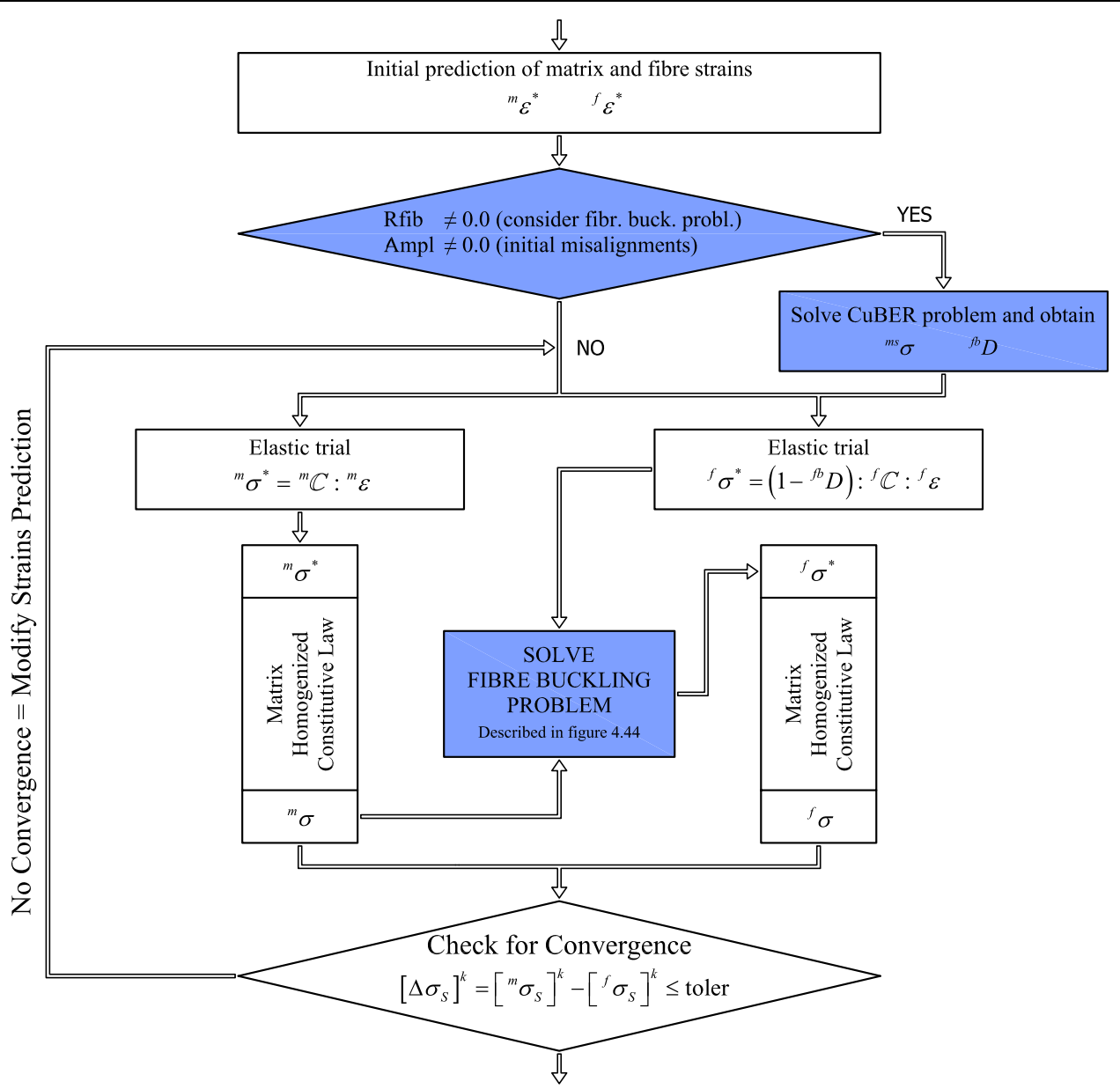

stress for straight fibers. If fiber stress is smaller than the buckling stress, the code leaves the algorithm. On the other hand, if fiber stress is larger than fiber buckling stress, fiber buckling occurs and the new fiber geometry is computed, so the code can solve the misaligned fiber problem (algorithm in Fig. 35).

The case in which the fibers enter the fiber buckling algorithm with some misalignment, either initial misalignment or a misalignment produced by fiber buckling of straight fibers, the code verifies if the parallel strain of fiber material is a compression or a tension strain. When a tensile load is applied to misaligned fibers, the micro-structural displacement suffered by them implies a reduction of their misalignment, transforming their curved geometry into a straight one. Thus, if the strain applied is a tensile strain, curved fibers are transformed into straight fibers by defining their amplitude value as 0.0. If fibers are compressed, the problem of misaligned fibers is solved. The flow chart used to solve this problem is shown in Fig. 35.

This last flow chart shows the algorithm developed to obtain the compression strength of fibers with initial misalignments. The CuBER problem is solved to obtain the structural damage parameter of fiber material. The fiber struc- tural stiffness used to calculate the structural damage parameter must be larger than the structural stiffness obtained if matrix is not confining fibers any more. Having calculated fiber structural stiffness, the new parallel stress prediction of fibers is compared with the buckling stress of a curved bar in an elastic foundation. If fibers buckle $\left({ }^{f} \sigma>\sigma_{c r}\right)$ the new buckled geometry is calculated and the problem is solved again. Otherwise, the prediction of fiber stresses are considered correct and the code leaves the algorithm after modifying the amplitude, wave-length and matrix structural stresses according to the scale factor defined in (81).

6.5 Numerical Performance of the Procedure Developed to Obtain the Compression Strength of Composite Materials. Numerical Examples

This section illustrates the numerical performance of the new procedure developed to obtain the compression strength of composite materials. With this aim, different numerical simulations of a RVE cell are studied. In each simulation some parameters are modified to obtain different RVE behaviors, that provide a better comprehension of how the numerical procedure developed works and the results that are 
Fig. 34 Fiber buckling

algorithm included in the serial/parallel mixing theory.

The procedure shaded in blue is described in the flow chart shown in Fig. 35

Fig. 35 Flow-chart description of the numerical procedure used for misaligned fibers
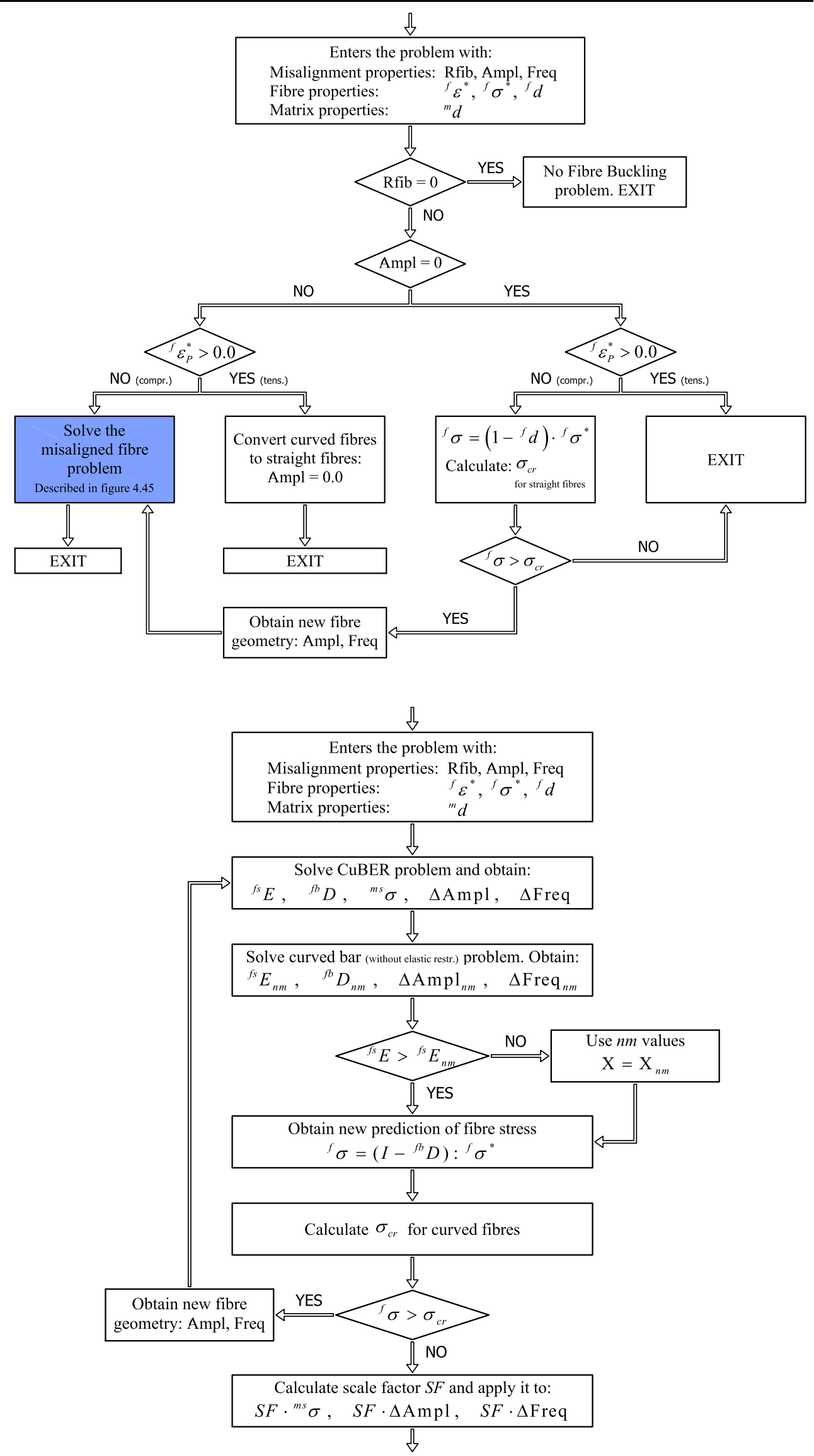


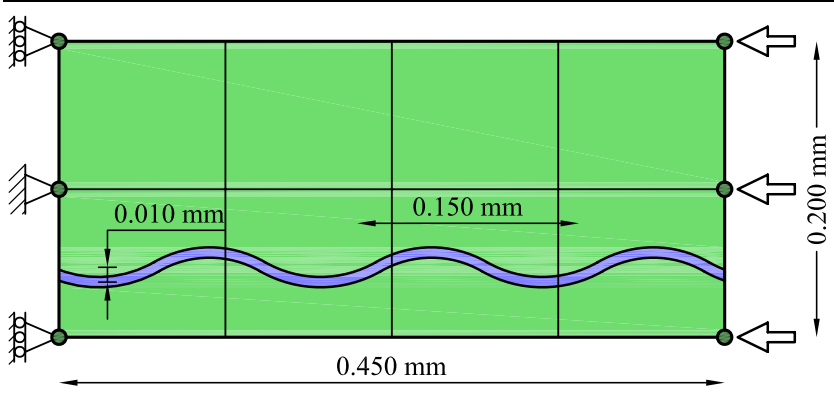

Fig. 36 Geometry and mesh of the RVE used in the numerical simulations to study the procedure developed to obtain the composite compression strength

Table 4 Fiber and matrix mechanical properties used to study the performance of the numerical procedure developed to obtain the compression strength of composites

\begin{tabular}{lc}
\hline Matrix Properties & \\
Elastic Modulus & $2.00 \mathrm{GPa}$ \\
Poisson Modulus & 0.30 \\
Compression Strength & $50.00 \mathrm{MPa}$ \\
Fracture Energy & $0.35 \mathrm{~kJ} / \mathrm{m}^{2}$ \\
Volume fraction & 0.50 \\
Fiber Properties & \\
Elastic Modulus & $70.00 \mathrm{GPa}$ \\
Poisson Modulus & 0.00 \\
Compression Strength & $2400.00 \mathrm{MPa}$ \\
Fracture Energy & $50.00 \mathrm{~kJ} / \mathrm{m}^{2}$ \\
Volume fraction & 0.50 \\
Fiber diameter & $7.00 \mu \mathrm{m}$ \\
\hline
\end{tabular}

expected when performing numerical simulations of composite materials.

The RVE cell used to perform the different numerical simulations included in this section has the geometry shown in Fig. 36 and the material properties used for all simulations, except specified otherwise, are shown in Table 4. Both materials, fiber and matrix, are defined as isotropic materials (the composite anisotropy is provided by the serial/parallel mixing theory). The compression force applied to the RVE is obtained with a displacement in the right nodes shown in Fig. 36.

The numerical examples shown in this section can be classified in different groups. The first group compares the performance of the composite when fibers have initial misalignments, when fibers are straight and when the fiber buckling problem is not considered. This simulation is followed by another one that compares the performance of the formulation based on the level of fiber initial misalignments. Afterwards the influence of matrix stiffness is studied in the case of straight fibers and in the case of fibers with initial misalignments. Next group verifies that

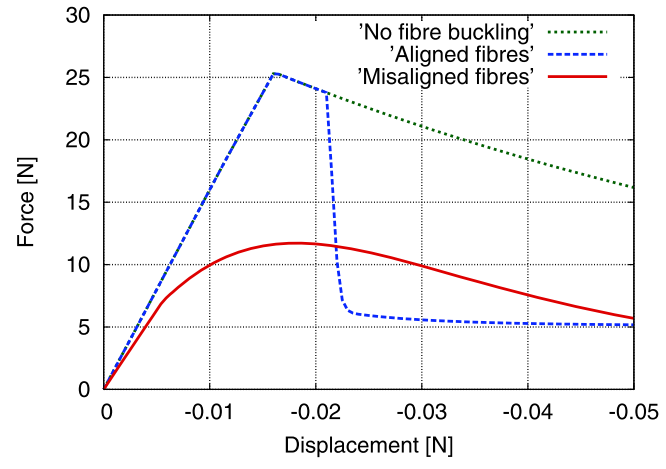

Fig. 37 Comparison among the results obtained when fibers have original misalignments, when fibers are straight and when the fiber buckling problem is not considered

the results obtained with the numerical procedure developed are mesh independent. Finally, the performance of the composite is studied for different loading-unloading cases.

\subsubsection{Comparison of the Compression Strength Obtained When Fibers Have or Do Not Have Initial Misalignments}

In this section the performance of the RVE cell shown in Fig. 36, with fiber initial misalignments, is compared with the performance obtained when fibers do not have initial misalignments and when the formulation developed to obtain the compression strength of the composite is not considered. This comparison provides a good understanding of the effects of fiber misalignments and of the reduction of the composite compression strength obtained due to fiber buckling.

Figure 37 shows the force-displacement response of the different RVE simulated for this comparison. This figure shows that fiber initial misalignments reduce the compression strength of the composite in more than a $50 \%$. On the other hand, in this case, having aligned fibers does not reduce the compression strength of the composite, as fiber damage appears before the buckling stress is reached. However, the fiber buckling formulation for straight fibers provides a different mechanical performance once the maximum compression load has been reached: fiber buckling occurs for an applied displacement of $22 \mu \mathrm{m}$, when matrix reach a damage level in which it cannot restrain fibers anymore.

In Figs. 38a and 38b is depicted the evolution of fiber and matrix structural damage, respectively. This figure shows that if fibers are straight, fiber buckling produces an instantaneous increase of the structural damage of both composite components. Furthermore, the resultant stiffness in straight fibers after buckling is lower than the stiffness obtained for the same deformation if fibers have initial misalignments. 


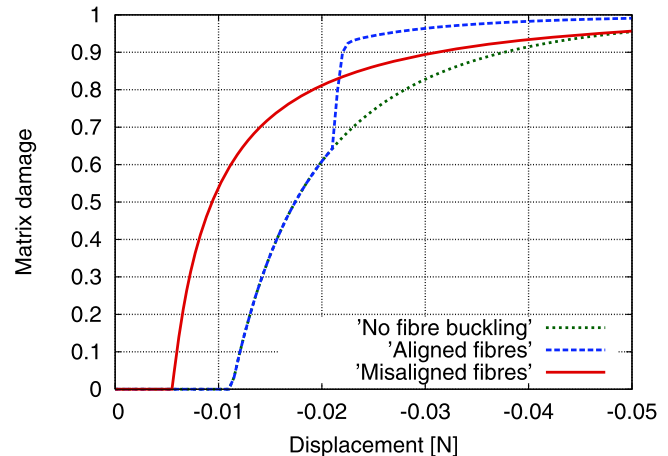

(a) Damage in matrix

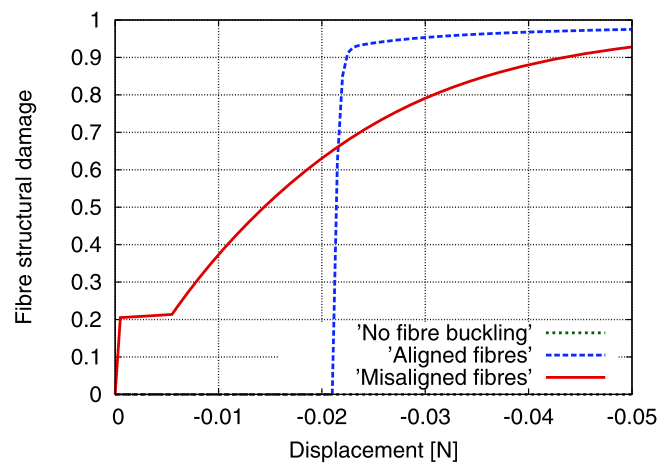

(b) Structural damage in fiber

Fig. 38 Evolution of matrix and fiber damage in the case of fibers with initial misalignments, straight fibers and when the fiber buckling problem is no considered

This effect is also shown in Fig. 37 and in Fig. 38, where the structural damage in straight fibers is larger than in misaligned ones. This result leads to the conclusion that, although a composite with straight fibers can reach larger loads, its structural behavior is more brittle than the one obtained in a composite with initial misalignments.

\subsubsection{Misaligned Fibers: Dependence on the Level of Initial Misalignment}

In this section the performance of two different RVE simulations containing fibers with initial misalignments is studied. The initial misalignments considered are defined varying the wavelength shown in Fig. 36. Their mechanical performance is compared also with the results obtained for the RVE cell when the compression strength formulation is not used. To perform these simulations, the compression strength of fiber material has been increased to $4500 \mathrm{MPa}$.

Two different wavelength values have been considered to compare the effects of the level of initial misalignment in fiber materials. One of the RVE models has an initial wavelength of $150 \mu \mathrm{m}$ while the other has a wavelength of $350 \mu \mathrm{m}$. Thus, using the quotient between amplitude and wavelength, the misalignments defined for the model with a wavelength of $150 \mu \mathrm{m}$ are 2.3 times more pronounced than in model with a wavelength of $350 \mu \mathrm{m}$.

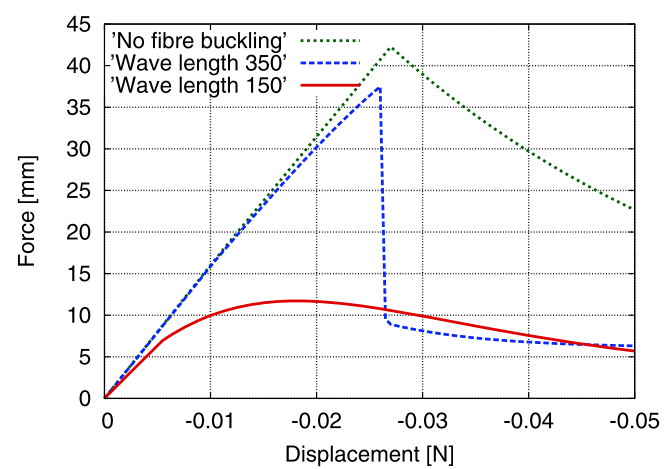

(a) Force-displacement

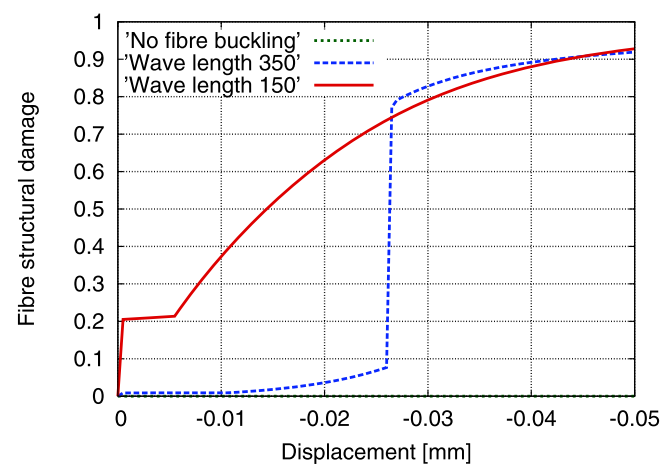

(b) Structural damage in fiber

Fig. 39 Composite performance for different levels of fiber initial misalignments

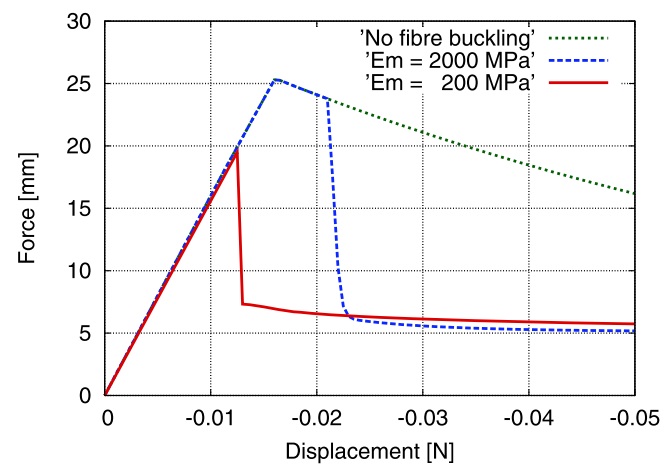

Fig. 40 Fiber buckling dependence on matrix confinement capacity in composites with initially aligned fibers

Figure 39a shows the force displacement graphs obtained for the different simulations performed. This graph shows that if fiber initial misalignments are reduced, the composite performance is more similar to the performance obtained when no misalignments are considered. Therefore, the critical load for the simulation with a wavelength of $350 \mu \mathrm{m}$ is reached when fiber buckles and not because the misalignment level reaches the maximum load that can be taken by the composite. The effect of fiber buckling in the simulation with a wavelength of $350 \mu \mathrm{m}$ can be seen more clearly studying the fiber structural damage parameter (Fig. 39b). 
Fig. 41 Finite element models used to prove the mesh independency of the new procedure developed

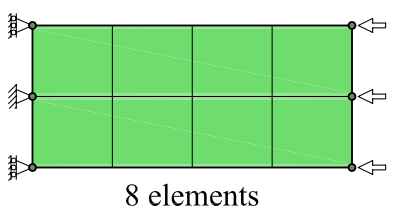

8 elements

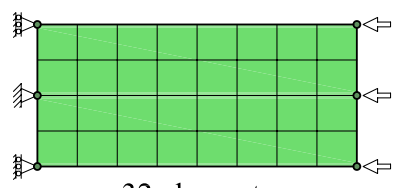

32 elements

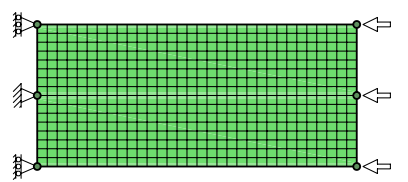

512 elements
Fiber buckling occurs when the damage parameter increases suddenly, for an applied displacement of $26 \mu \mathrm{m}$, varying its value from 0.08 (value obtained due to the initial misalignment defined) to 0.8 .

\subsubsection{Aligned Fibers. Effect of Matrix Confinement}

This section shows the effect of matrix confinement in composites in which fibers do not have initial misalignments. Matrix confinement is directly proportional to matrix stiffness, therefore, two different simulations of the RVE cell described in Fig. 36 have been performed with two different matrix stiffnesses.

All material properties considered in these simulations correspond to the properties defined in Table 4, with the exception of matrix young modulus. The matrix defined in one of the simulations has a young modulus of ${ }^{m} E=2000 \mathrm{MPa}$ while, in the other simulation, the matrix material defined has a young modulus of ${ }^{m} E=200 \mathrm{MPa}$. Thus, the confinement capacity of this last matrix is reduced ten times compared to the first matrix defined.

The composite performance obtained for these two simulations is shown in the force displacement graph displayed in Fig. 40. This graph shows that while the maximum compression load is defined by fiber compression strength when matrix is strongly confining fibers (case of ${ }^{m} E=2000 \mathrm{MPa}$ ), if this confinement capacity is reduced by ten fiber buckles before reaching its maximum compression stress.

These simulations prove the ability of the numerical procedure developed to take into account the effect of matrix confinement when no fiber misalignments have been defined in the composite. This ability is rather important when simulating composite structures under cyclic loads. In these cases, matrix degradation increases due to fatigue, its stiffness is reduced and, at a certain number of cycles, a sudden fracture of the composite can occur due to fiber buckling.

\subsubsection{Dependence on Mesh Refinement}

One of the main problems found when fracture mechanics is applied to the finite element method is that, in most cases, the results become mesh dependent. This happens because a continuum formulation (fracture mechanics) is being used in a discrete procedure (finite elements). In a damage formulation, the mesh independency is obtained with the definition of the fracture length parameter $[58,63]$.

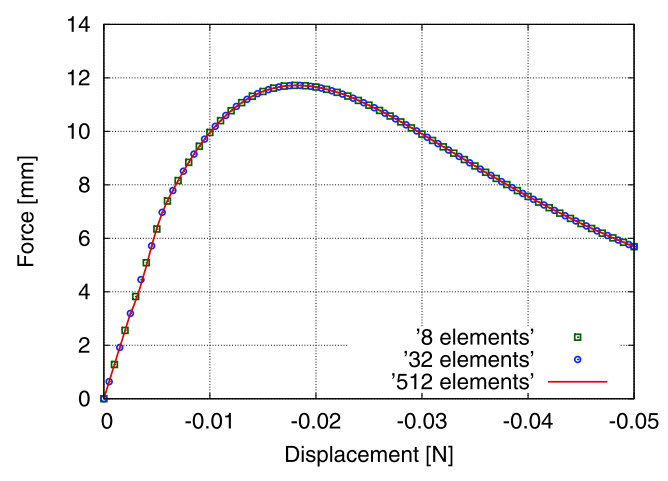

Fig. 42 Force displacement graph for the different simulations performed

In the numerical procedure developed to obtain the compression strength of composite materials, the fracture length has been re-defined when formulating the matrix homogenized constitutive law (Sect. 4), in order to adjust the physical meaning of fracture energy to the parameters included in the new constitutive equation. The fracture length of the matrix affected by fiber misalignments depends of the wavelength of these misalignments, instead of depending on the finite element size.

To prove the validity of the new fracture length considered, three different numerical simulations of the RVE cell defined in Fig. 36 have been performed, with three different meshes. Each mesh has a different number of finite elements: 8, 32 and 512. Figure 41 shows the finite element models developed.

The results provided by the three different simulations are exactly the same, as proves the force displacement graph shown in Fig. 42. This agreement among the results obtained for all simulations shows the validity of the fracture length value defined for the new homogenized constitutive equation of matrix material.

\subsubsection{Numerical Performance of the Formulation for a Load-Unload Path}

The last group of simulations conducted consist on loading and unloading the different RVE models.

The main objective of these simulations is to show the performance of the algorithm developed when the composite is unloaded. The first results that are shown correspond to the composite in which fibers have initial misalignments. 


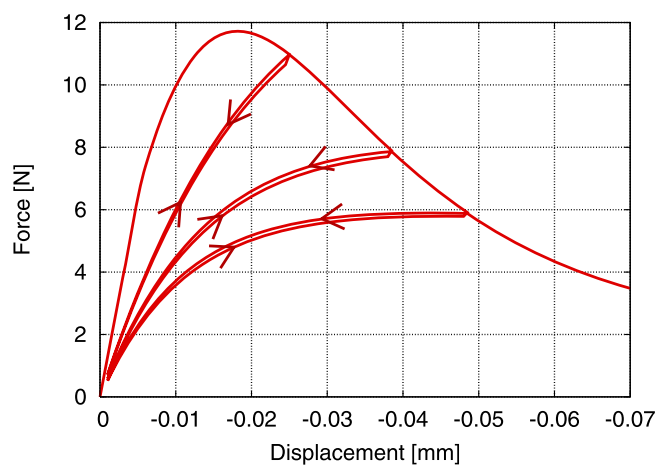

Fig. 43 Composite with misaligned fibers under a load-unload path. Force-displacement graph

Afterwards, the load path is applied to the RVE with straight fibers.

The composite performance is studied using a forcedisplacement graph, which shows the maximum compression load that can be applied to the composite and its postcritical behavior once the maximum load has been reached. The force displacement graph obtained for the RVE cell with misaligned fibers is shown in Fig. 43.

This graph shows that the unloading branches are not straight (as usually happens with a damage formulation) but, instead, the composite stiffness increases as the load is being reduced. This effect is produced by fiber misalignments: as the load is reduced, fiber misalignments are also reduced, increasing their stiffness.

This effect leads to a damage performance for the composite in which damage is recovered if the material is unloaded. Thus, unlike the damage formulation used in continuum mechanics [57,63], the structural damage due to fiber buckling does not remain constant in the structure. However, although composite stiffness is recovered, when it is compressed again the load does not follow the elastic branch but, instead, follow the path defined in the unloading process (Fig. 43). To explain this effect is useful plot the damage evolution of fiber and matrix, which are shown in Fig. 44.

As can be seen in Fig. 44, although damage in fiber is partially recovered (due to the reduction of fiber misalignments), matrix damage remains constant during the unloading process. Thus, when the compression load is applied again, the confinement capacity of matrix is lower than the confinement capacity provided by the matrix the first time that the composite was compressed, which leads to a faster increase of fiber initial misalignments.

If fibers do not have initial misalignments, the force displacement graph obtained for the RVE is the one displayed in Fig. 45. In this case, the first time the structure is unloaded fiber buckling has not yet occurred. Therefore, the unloading path and the following loading path have a constant stiffness. After fiber buckling, the performance of misaligned fibers is recovered: when the structure is unloaded it recovers its

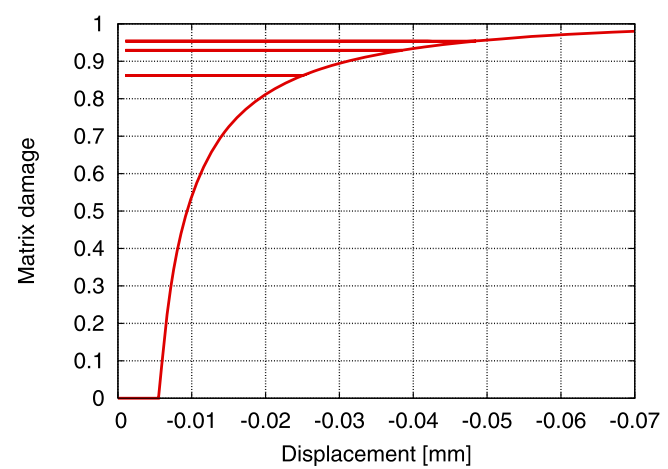

(a) Matrix damage

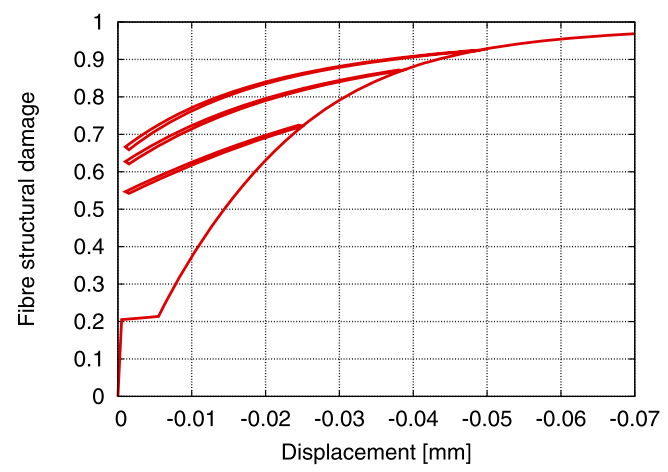

(b) Fiber structural damage

Fig. 44 Composite with misaligned fibers under a load-unload path. Evolution of damage in matrix and fiber

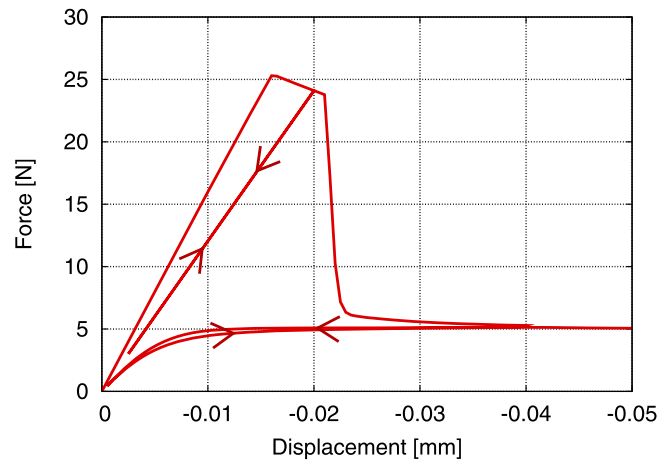

Fig. 45 Composite with initially straight fibers under a load-unload path. Force-displacement graph

stiffness and when it is loaded again, it follows the previous unload path.

\section{Validation of the Formulation Proposed and Numerical Example}

In this section the formulation proposed to obtain the compression strength of composite materials is validated comparing the results obtained from the numerical prediction with the experimental data. This validation proves the abil- 
Table 5 Compression strength of the samples that will be compared with the numerical results

\begin{tabular}{ll}
\hline Sample & $\sigma_{C}[\mathrm{MPa}]$ \\
\hline ACA & $560.90( \pm 35.1)$ \\
CAA & $477.74( \pm 36.6)$ \\
CBB & $521.56( \pm 16.2)$ \\
\hline
\end{tabular}

Table 6 Misalignment values and number of measurements performed by Barbero and Tomblin

\begin{tabular}{lll}
\hline Sample & $\begin{array}{l}\text { Half normal distribution } \\
\text { expected value (degrees) }\end{array}$ & $\begin{array}{l}\text { Number of } \\
\text { measurements }\end{array}$ \\
\hline ACA & 2.87 & 1359 \\
CAA & 2.76 & 1271 \\
CBB & 2.63 & 1224 \\
\hline
\end{tabular}

Table 7 Material components and volume fractions of the experimental samples used to validate the developed formulation

Sample: ACA

Matrix material:

2036C Polyester with styrene

Fiber material:

Glass fiber OC 102-AA-56

Fiber vol. fraction:

$55.2 \%$

Sample: CAA

Matrix material:

2036C Polyester

Fiber material

Glass fiber OC 102-AA-56

Fiber vol. fraction:

$40.2 \%$

Sample: CBB

Matrix material:

D-1419 Vinyl Ester

Fiber material:

Glass fiber OC 366-AD-113

Fiber vol. fraction:

$43.0 \%$

ity of the numerical procedure developed to obtain the compression strength of real composite materials due to the fiber buckling phenomenon. The experimental results used to validate the formulation have been obtained from Barbero and Tomblin $[3,4]$.

\subsection{Description of the Experimental Test}

The experimental tests performed by Barbero and Tomblin $[3,4]$ where made over cylindrical rods, with a diameter of $9.2 \mathrm{~mm}$, pultruded by Creative Pultrusions Inc. The composite materials used were made of E-Glass fibers embedded in a polymeric matrix. Eleven different composites where tested, each one with a different combination of fiber material, matrix material and fiber volumetric participation. For each one of these materials, eight different compression tests were performed to obtain an average value of the compression strength for each one of them.
Table 8 Matrix mechanical properties

2036C Polyester

Elastic Modulus

$3.00 \mathrm{GPa}$

Poisson Modulus

0.35

Tensile Strength

$35.00 \mathrm{MPa}$

Fracture Energy

$0.17 \mathrm{~kJ} / \mathrm{m}^{2}$

D-1419 Vinyl Ester

Elastic Modulus

$3.50 \mathrm{GPa}$

Poisson Modulus

0.35

Tensile Strength

$40.00 \mathrm{MPa}$

Fracture Energy

$0.31 \mathrm{~kJ} / \mathrm{m}^{2}$

Table 9 Fiber mechanical properties

E-Glass OC-102-AA-56

Elastic Modulus

$80.00 \mathrm{GPa}$

Poisson Modulus

0.20

Tensile Strength

$1500.00 \mathrm{MPa}$

Fiber Diameter

$13.00 \mu \mathrm{m}$

E-Glass OC-366-AA-113

Elastic Modulus

$80.00 \mathrm{GPa}$

Poisson Modulus

0.20

Tensile Strength

$1500.00 \mathrm{MPa}$

Fiber Diameter

$23.00 \mu \mathrm{m}$

The compression tests were performed using short samples $(38 \mathrm{~mm})$ to prevent the global buckling of the sample. Strain gages with a length equal to the specimen size where applied to each sample. Half of the samples contained gages disposed back-to-back to verify the specimen alignment. The compression failure was observed at the center of the gage section for the $75 \%$ of the samples. The compression strength was obtained using a modified ASTM D-695 test fixture [3].

From all the tests performed by Barbero and Tomblin, three of them, chosen as the most representative, are used to validate the proposed formulation. Their mean compression strength value and the $95 \%$ confidence interval is displayed in Table 5.

Misalignment measurements were performed with the optical technique proposed by Yurgartis [50]. The number of measurements made to each sample was determined so that the expected value of the half normal distribution had a $95 \%$ confidence interval of $\pm 0.2^{\circ}$. The half normal distribution is considered because fiber micro-buckling occurs at the same load for positive or negative misalignment angles [3]. The misalignment angles of the samples under study are displayed in Table 6 .

The constituent materials, fiber and matrix, of each sample and their volumetric fractions are shown in Table 7. As 
the materials properties were not defined in [3], their value has been obtained as an average of the values defined in literature for polymeric matrices and E-Glass fibers. Matrix mechanical characteristics are described in Table 8. Table 9 describes the mechanical characteristics of fibers. Both materials have been simulated with a damage formulation.

\subsection{Description of the Numerical Simulation}

To validate the proposed formulation a numerical simulation of the three different samples previously described: ACA, $\mathrm{CAA}$ and $\mathrm{CBB}$, has been performed. The numerical model developed corresponds to a representative volume element (RVE) in which a two dimensional iso-strain formulation has been used. The geometry of the RVE considered is displayed in Fig. 46. This figure also shows the finite element mesh used to perform the simulation and the boundary conditions applied to it.

Three different numerical simulations have been performed corresponding to the three experimental samples used to validate the formulation. The composite material defined in each simulation correspond to the composites defined in Table 7. The properties of the matrix and fiber materials are the ones shown in Tables 8 and 9, respectively.

In order to take into account fiber initial misalignments in the calculation of the compression strength of the composite, three different parameters are required: fiber radius, misalignment amplitude and misalignment wave-length. Fiber radius is a known parameter dependent on the fibers used and its value is defined in Table 9. Misalignment values are obtained from values provided by Barbero and Tomblin for the half normal distribution expected misalignment angle (Table 6).

The angle defined in Table 6 is defined, in the numerical simulation, as the angle found in the inflection point of the

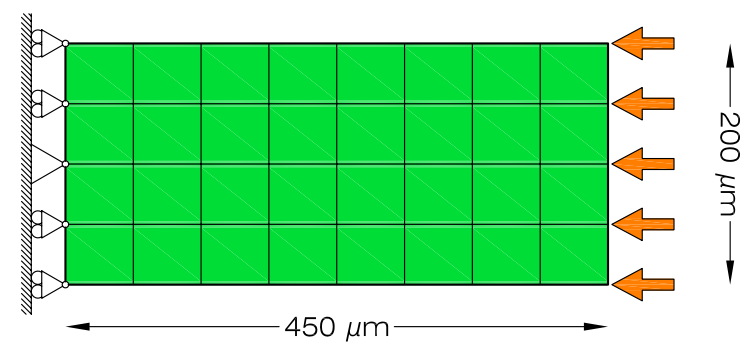

Fig. 46 Geometry and mesh defined for the simulation used to validate the formulation developed fiber geometry (point A in Fig. 47). This angle is the largest angle found in the sinusoidal geometry of the fiber.

The second parameter used to define the geometry of the fiber has been the misalignment amplitude, that has been defined using the statement made by Jochum and Grandidier [15], who define the amplitude as one to two times the fiber diameter. Once having defined the misalignment amplitude, using the value of fiber diameter, the wave-length can be computed from the misalignment angle defined by Barbero and Tombin as:

$\lambda=2 A \frac{\sin \alpha}{1-\cos \alpha}$

with, $\lambda$ and $A$ the misalignment wave-length and amplitude (respectively) and $\alpha$ the misalignment angle.

The misalignment parameters used to perform the finite element simulations are the ones displayed in Table 10 for each sample considered.

\subsection{Comparison Among the Experimental and the Numerical Results}

The comparison between the experimental and the numerical results is made with the force-displacement graphs obtained from the numerical simulation. The force applied to the finite element structure is plotted against the displacement in the face where the load is applied. The stresses provided by Barbero and Tomblin are transformed into the force that would resist the RVE defined in Fig. 46.

The maximum experimental compression strength obtained for each composite is represented, in Fig. 48, with an horizontal line. The numerical results are represented as force-displacement graphs. This figure shows the agreement between the maximum compression strength obtained with the formulation developed and the experimental results.

The maximum error obtained with the numerical simulations correspond to the ACA sample and is lower than $2.6 \%$. Also, it is worth to point out that the error obtained with all

Table 10 Misalignment parameters defined in the finite element models used to validate the developed formulation

\begin{tabular}{llll}
\hline Sample & $\varnothing_{\text {fib }}$ & $A$ & $\lambda$ \\
\hline ACA & $13 \mu \mathrm{m}$ & $15.0 \mu \mathrm{m}$ & $1198 \mu \mathrm{m}$ \\
CAA & $13 \mu \mathrm{m}$ & $15.0 \mu \mathrm{m}$ & $1246 \mu \mathrm{m}$ \\
CBB & $23 \mu \mathrm{m}$ & $25.0 \mu \mathrm{m}$ & $2175 \mu \mathrm{m}$ \\
\hline
\end{tabular}

Fig. 47 Fiber misalignment and misalignment angle considered

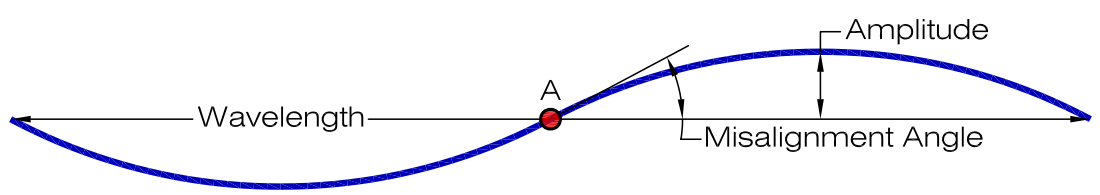




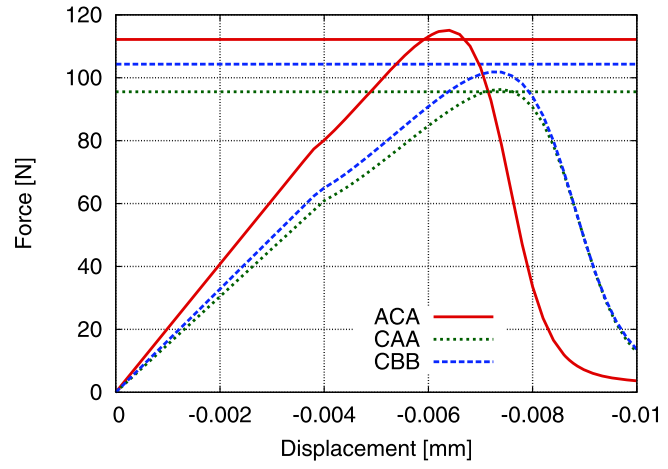

Fig. 48 Comparison among the experimental and the numerical compression stress obtained for each sample

Table 11 Maximum compression load of the experimental and numerical samples and error obtained with the numerical simulations

\begin{tabular}{lllll}
\hline Sample & $\begin{array}{l}\text { Experimental } \\
\text { F max }\end{array}$ & Numerical & \\
\cline { 3 - 5 } & {$[\mathrm{N}]$} & \multicolumn{1}{l}{$\begin{array}{l}\text { F max } \\
{[\mathrm{N}]}\end{array}$} & $\begin{array}{l}\text { Error } \\
{[\mathrm{N}]}\end{array}$ & $\begin{array}{l}\text { Error } \\
{[\%]}\end{array}$ \\
\hline ACA & $112.18( \pm 7.02)$ & 115.09 & 2.91 & $2.6 \%$ \\
CAA & $95.55( \pm 7.32)$ & 96.29 & 0.74 & $0.8 \%$ \\
CBB & $104.31( \pm 3.24)$ & 101.84 & -2.47 & $-2.4 \%$ \\
\hline
\end{tabular}

numerical models is inside the $95 \%$ confidence interval obtained with the experimental data. The maximum compression values, as well as the errors obtained with each model are displayed in Table 11.

Figure 48 shows that when the composite reaches the maximum compression load, its post-critical strength falls abruptly. This is because the fiber buckling phenomenon. The maximum compression load is reached when matrix cannot restrain fiber micro-structural deformation any more (for an applied displacement of $0.006 \mathrm{~mm}$, matrix damage is nearly 1.0). At this point fiber structural damage increases exponentially leading to the reduction of the compression load that can be applied to the composite material. This phenomenon is shown in Fig. 49, in which the fiber and matrix structural damage parameters are plotted against the compression displacement applied to the RVE that simulates the ACA sample.

The developed formulation adds two different structural stresses to matrix material to increase its damage due to the fiber-matrix micro-structural interaction (see Sect. 4). Of these two stresses the one that leads to the matrix damage shown in Fig. 49 is the one that increase the shear stresses in matrix material. This result is important as it agrees with all existing theories dealing with the fiber buckling problem $[1,4,8,10]$, in which the compression strength of the composite has a strong dependence on the shear strength and stiffness of matrix material.

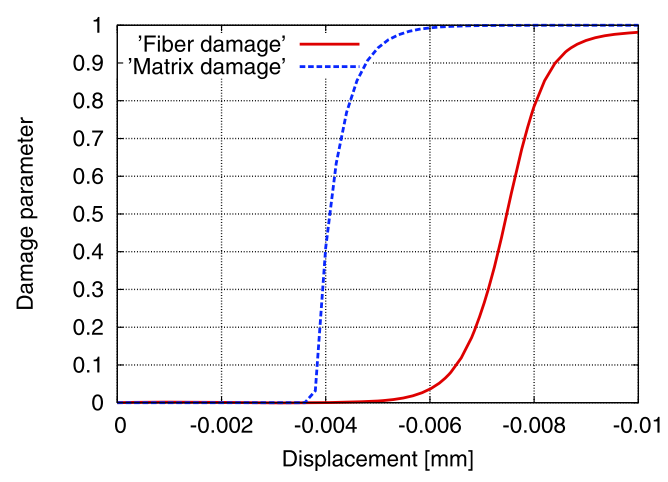

Fig. 49 Structural damage in fiber and matrix materials. ACA sample

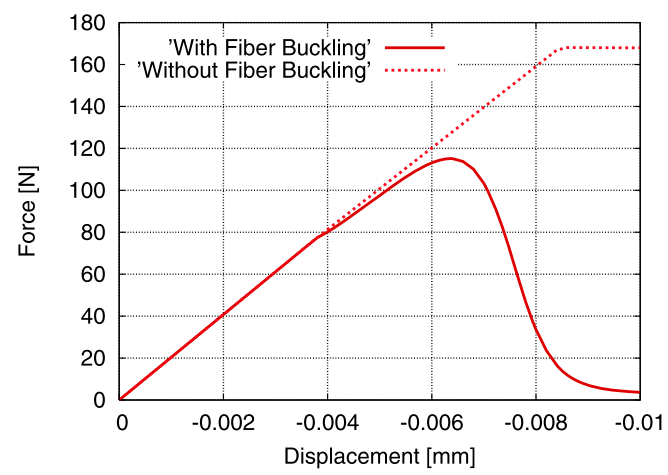

Fig. 50 Comparison of the results obtained when the fiber buckling formulation is or is not considered in the numerical simulation. ACA sample

It is also interesting to compare the RVE response that is obtained if the compression strength algorithm is not used in the simulation. Figure 50 shows the performance of the ACA sample when it is simulated using the compression strength formulation and when this formulation is not considered. The composite performance is shown with a forcedisplacement graph.

This last figure shows that, when matrix damage starts for an applied displacement of $0.004 \mathrm{~mm}$ (Fig. 49), the composite stiffness is slightly reduced if compared to the simulation in which the fiber buckling formulation is not used. Figure 50 also shows that the reduction in the compression capacity obtained with the developed formulation is larger than a $30 \%$.

With these results, it can be concluded that the formulation developed to obtain the compression strength of composite materials can reproduce experimental results of real composites. Also, the reduction of the compression capacity in the composite is large enough to be taken into account.

\section{Conclusions}

This paper reviews the different procedures existing in literature to obtain the compression strength of long fiber com- 
posites, due to the effect of fiber micro-buckling; and proposes a new formulation capable of obtaining the maximum compression stress that can be applied to the composite, and the post-critical performance of the material once fiber buckling has taken place. This last achievement is important because, when performing numerical simulations of structures, the failure of an element does not always imply the failure of the whole structure. Therefore, the post-critical strength of the element is required to continue the calculation.

The formulation presented has been developed taking into account that its implementation is made in a finite element code (PLCd) that deals with composite materials using the serial/parallel mixing theory. During the process of defining the formulation proposed, the characteristics of these two numerical methods have been always taken into consideration, in order to take advantage of all possibilities and information provided by them. Following this approach, the final formulation does not consist of an equation (or a set of equations) that provides the maximum stress that can be applied to the composite. Instead, it establish a relation between the mechanical performance of the composite components that leads, straightforward, to the result sought: the maximum compression that can be applied to the composite due to the fiber buckling phenomenon.

The compression strength formulation has been obtained defining an homogenization of the constitutive performance of fiber and matrix materials. This homogenization introduces the micro-structural interaction between these two composite components into their constitutive equations. The homogenized constitutive equations have been introduced in an algorithm that interacts with the serial/parallel mixing theory, taking into account all possible situations that can be found in a long fiber reinforced composites, from loading paths to level of initial misalignment in the fibers.

The study of the numerical procedure developed has proved that the formulation has a strong dependence on the three parameters in which are based all formulations dealing with the fiber buckling problem (since the first approach proposed by Rosen [1]): fiber initial misalignment, shear strength of matrix material and volumetric participation of fiber and matrix in the composite. The dependence on these three parameters is important because it proves that the formulation developed agrees with the existing knowledge on the field. Besides this agreement, the study of the formulation has also shown that the expected mechanical behavior of composites under compression forces is well represented by the numerical procedure proposed. In the following are described the most significant mechanical responses of compressed composites, reproduced by the developed formulation.

- The damage obtained in the composite due to fiber buckling is partially recoverable. Damage found in fibers, as a result of buckling, can be recovered if the composite is unloaded, because fibers recover their original alignment. However, the damage suffered by matrix as a result of the efforts applied to it, which are increased by the microstructural interaction between fibers and matrix, is not recoverable. Therefore, although damage in fiber is recovered, damage in matrix not, so fiber buckling occurs for lower loads is the composite is loaded again.

- When fiber initial misalignments are too small or nonexistent, the compression load that can be applied to the composite is substantially larger. However, in these cases, fiber buckling is followed by a sudden lost of strength in the composite, obtaining a brittle failure of the material. This performance agrees with the buckling performance obtained in any structural member: the new geometry adopted by the buckled structure produces a sudden reduction of the longitudinal length of the element, increases the second order effects and leads to a dramatic reduction of the structure stiffness.

- The brittle failure obtained in the case of initially aligned fibers provides a lower post-critical strength in the composite, compared to the case of initially misaligned fibers. In this last case, the post-critical path followed by the composite is smoother because the misalignment in fibers is smaller (the quotient between the amplitude and the wave-length is smaller). Having smaller misalignments provides stiffer fibers and reduces the structural damage in matrix due to the fiber-matrix interaction.

- The mechanical performance obtained in fibers with small misalignments (large wave-lengths with small amplitudes) is very similar to the mechanical performance obtained in fibers that are considered straight. The effects of fiber buckling are the same obtained for initially straight fibers: brittle failure and smaller post-critical strength.

- Matrix stiffness has an important role in fiber buckling phenomenon by restraining fibers from buckle. If matrix stiffness is reduced (due to fatigue or because it has been damaged due to tensile stresses applied before compressing the composite), its confinement capacity is also reduced and fiber buckling will occur for lower loads. This effect is obtained independently of the level of initial misalignment found in fibers.

Finally, it can be concluded that the validation performed of the formulation developed, comparing numerical with experimental results, has proved the ability of the formulation to simulate real composites and to obtain their compression strength. This validation has also shown the necessity of taking into account the fiber buckling phenomenon in compressed composites, as the maximum compression stress that can be applied to it is lower than a $30 \%$, compared with the maximum compression that can be applied if the algorithm developed is not used. 


\section{References}

1. Rosen B (1965) Fibre composite materials. Am Soc Metals, Metals Park, pp 37-45

2. Rastellni F, Oller S, Salomon O, Oñate E (2008) Composite materials non-linear modelling for long fibre reinforced laminates: Continuum basis, computational aspects and validations. Comput Struct 86(9):879-896

3. Barbero EJ, Tomblin JS (1996) A damage mechanics model for compression strength of composites. Int J Solids Struct 33(29):4379-4393

4. Tomblin JS, Barbero EJ, Godoy LA (1997) Imperfection sensitivity of fiber micro-buckling in elastic-nonlinear polymer-matrix composites. Int J Solids Struct 34(13):1667-1679

5. Welsh JS, Adams DF (1997) An experimental investigation of the mini-sandwich laminate as used to obtain unidirectional composite compression strengths. J Compos Mater 31(3):293-314

6. Barbero EJ (1998) Prediction of compression strength of unidirectional polymer matrix composites. J Compos Mater 32(5):483502

7. Balacó de Morais A (1996) Modelling lamina longitudinal compression strength of carbon fibre composite laminates. J Compos Mater 30(10):1115-1131

8. Balacó de Morais A, Torres Marques A (1997) A micromechanical model for the prediction of the lamina longitudinal compression strength of composite laminates. J Compos Mater 31(14):13971412

9. Balacó de Morais A (2000) Prediction of the layer longitudinal compression strength. J Compos Mater 34(21):1808-1820

10. Drapier S, Grandidier JC, Potier-Ferry M (1999) Towards a numerical model of the compressive strength for long fibre composites. Eur J Mech A, Solids 18(1):69-92

11. Drapier S, Grandidier JC, Potier-Ferry M (2001) A structural approach of plastic microbuckling in long fibre composites: comparison with theoretical and experimental results. Int J Solids Struct 38(22-23):3877-3904

12. Parnes R, Chiskis A (2002) Buckling of nano-fibre reinforced composites: a re-examination of elastic buckling. J Mech Phys Solids 50(4):855-879

13. Akbarov SD, Kosker R (2001) Fiber buckling in a viscoelastic matrix. Mech Compos Mater 37(4):299-306

14. Kosker R, Akbarov SD (2003) Influence of the interaction between two neighboring periodically curved fibres on the stress distribution in a composite material. Mech Compos Mater 39(2):165176

15. Jochum C, Grandidier JC (2004) Microbuckling elastic modelling approach of a single carbon fibre embedded in an epoxy matrix. Compos Sci Technol 64(16):2441-2449

16. Puig JM, Car E, Oller S (2001) Solution for the inelastic buckling of long fibre reinforced composite materials. In: Figuieras J, Juvanades L, Farias R, Balkema A (eds) CCC 2001—Composites in construction international conference, pp 119-124

17. Sánchez-Palencia E (1987) Homogenization techniques for composite media. In: Boundary layers and edge effects in composites. Springer, Berlin, pp 121-192

18. Oller S, Miquel J, Zalamea F (2005) Composite material behaviour using a homogenization double scale method. J Eng Mech 131(1):65-79

19. Gónzalez C, Llorca J (2007) Mechanical behavior of unidirectional fiber-reinforced polymers under transverse compression: Microscopic mechanisms and modeling. Compos Sci Technol 67(5):2795-2806

20. Zalamea F (2001) Tratamiento numérico de materiales compuestos mediante la teoría de homogeneización. Ph.D. thesis, Departament de Resistència de Materials i Estructures a l'Enginyeria (RMEE)_UPC. Directors: Juan Miquel Canet and Sergio Oller
21. Car E, Zalamea F, Oller S, Miquel J, Oñate E (2002) Numerical simulation of fiber reinforced composites - two procedures. Int J Solids Struct 39(7):1967-1986

22. Trusdell C, Toupin R (1960) The classical field theories. Handbuch der Physik, vol. 3(1). Springer, Berlin

23. Ortiz M, Popov E (1957) A physical model for the inelasticity of concrete. Proc R Soc Lond, Ser A 383:101-125

24. Ortiz M, Popov E (1982) Plain concrete as a composite material. Mech Mater 1:139-150

25. Green A, Naghdi P (1965) A dynamical theory of interacting continua. J Eng Sci 3:231-241

26. Oller S, Oñate E, Miquel J, Botello S (1996) A plastic damage constitutive model for composite materials. Int J Solids Struct 33(17):2501-2518

27. Car E (2000) Modelo constitutivo continuo para el estudio del comportamiento mecánico de los materiales compuestos. Ph.D. thesis, Departament de Resistència de Materials i Estructures a l'Enginyeria (RMEE)—UPC. Directors: Sergio Oller and Eugenio Oñate

28. Malvern LE (1968) Introduction to the mechanics of a continuous medium. Prentice-Hall, Englewood Cliffs

29. Lubliner J (1990) Plasticity theory. MacMillan \& Co., New York

30. Oller S (2002) Dinámica no-lineal. CIMNE (Centro Internacional de Métodos Numéricos en Ingeniería), Barcelona

31. Car E, Oller S, Oñate E (2000) An anisotropic elasto plastic constitutive model for large strain analysis of fiber reinforced composite materials. Comput Methods Appl Mech Eng 185(2-4):245-277

32. Car E, Oller S, Oñate E (2001) A large strain plasticity model for anisotropic materials - composite material application. Int J Plast 17(11):1437-1463

33. Oller S, Neamtu L, Oñate E (1995) Una generalización de la teoría de mezclas clásica para el tratamiento de compuestos en serie/paralelo. In: París F, Cañas J (eds) MATCOMP 95, pp 433438

34. Neamtu L, Oller S, Oñate E (1997) A generalized mixing theory elasto-damage-plastic model for finite element analysis of composites. In: Owen D, Oñate E, Hinton E (eds) Complas VComputational plasticity, pp 1-5

35. Rastellini F, Oller S, Salomon O, Oñate E (2003) Advanced serialparallel mixing theory for composite materials analysis. Continuum basis and finite element applications. In: Oñate E, Owen D (eds) COMPLAS 2003-Proceeding CD of the VII international conference on computational plasticity

36. Salomon O, Rastellini F, Oller S, Oñate E (2005) Fatigue prediction for composite materials and structures. In: Research N, technology organisation (RTO) (eds) Air vehicle technology: AVT121. Symposium on the evaluation, control and prevention of high cycle fatigue

37. Rastellini F (2006) Numerical modelling of the constitutive nonlinearity of composite laminates. Ph.D. thesis, Departament de Resistència de Materials i Estructures a l'Enginyeria (RMEE)UPC. Directors: Sergio Oller and Eugenio Oñate

38. Martínez X, Oller S, Rastellini F, Barbat A (2008) A numerical procedure simulating RC structures reinforced with FRP using the serial/parallel mixing theory. Comput Struct 86(15-16):16041618

39. Martínez X, Oller S, Barbero E (2008) Study of delamination in composites by using the serial/parallel mixing theory and a damage formulation. In: Mechanical response of composites. Computational methods in applied sciences. Springer, Berlin

40. CIMNE: PLCd Manual. Non-linear thermomechanic finite element code oriented to $\mathrm{PhD}$ student education (1991-2008). Finite element code developed at CIMNE

41. Oller S, Oñate E, Oliver J, Lubliner J (1990) Finite element non-linear analysis of concrete structures using a plastic-damage model. Eng Fract Mech 35(1-3):219-231 
42. Lubliner J, Oliver J, Oller S, Oñate E (1989) A plastic-damage model for concrete. Int J Solids Struct 25(3):299-326

43. Barbat AH, Oller S, Oñate E, Hanganu A (1997) Viscous damage model for Timoshenko beam structures. Int J Solids Struct 34(30):3953-3976

44. Mata P, Oller S, Barbat AH (2007) Static analysis of beam structures under nonlinear geometric and constitutive behaviour. Comput Methods Appl Mech Eng 196(45-48):4458-4478

45. Mata P, Oller S, Barbat AH (2008) Dynamic analysis of beam structures considering geometric and constitutive nonlinearity. Comput Methods Appl Mech Eng 197(6-8):857-878

46. Jones RM (1999) Mechanics of composite materials, 2nd edn. Taylor \& Francis, Philadelphia

47. Timoshenko SP, Gere JM (1961) Theory of elastic stability, international student edn. McGraw-Hill, Tokyo

48. Lo KH, Chim ESM (1992) Compressive strength of unidirectional composites. J Reinf Plast Compos 11(8):838-896

49. Xu YL, Reifsnider KL (1993) Micromechanical modeling of composite compressive strength. J Compos Mater 27(6):558-572

50. Yurgartis SW (1987) Measurement of small angle fiber misalignment in continuous fiber composites. Compos Sci Technol 30(4):279-293

51. Paluch B (1996) Analysis of geometric imperfections affecting the fibers in unidirectional composites. J Compos Mater 30(4):454 485

52. Clarke AR, Archenhold G, Davidson NC (1995) A novel technique for determining the $3 \mathrm{~d}$ spatial distribution of glass fibers in polymer composites. Compos Sci Technol 55(1):75-91

53. Wisnom M (2000) Size effects in Composites. In: Comprehensive composite materials, vol 5. Test methods, nondestructive evaluation and smart materials. Elsevier, London, pp 23-47

54. Grandidier JC, Ferron G, Potier-Ferry M (1992) Microbuckling and strength in long-fiber composites: theory and experiments. Int J Solids Struct 29(14-15):1753-1761

55. Guz AN, Lapusta YN (1999) Three-dimensional problems of the near-surface instability of fiber composites in compression (model of a piecewise-uniform medium)(survey). Int Appl Mech 35(7):641-670

56. Puig JM, Car E, Oller S (2002) Solución numérica para el pandeo inelástico de materiales compuestos reforzados con fibras largas.
In: Análisis y cálculo de estructuras de materiales compuestos. CIMNE (Centro Internacional de Métodos Numéricos en Ingeniería), Barcelona, pp 295-320

57. Kachanov LM (1986) Introduction to continuum damage mechanics. Mechanics of elastic stability. Nijhoff, Dordrecht

58. Oliver J, Cervera M, Oller S, Lubliner J (1990) Isotropic damage models and smeared crack analysis of concrete. In: Mang H, Bicánić N (eds) Second international conference on computer aided analysis and design of concrete structures, pp 945-958

59. Oller S (2003) Simulación numérica del comportamiento mecánico de los materiales compuestos. CIMNE (Centro Internacional de Métodos Numéricos en Ingeniería), Barcelona

60. Naik NK, Tiwari SI, Kumar RS (2003) An analytical model for compresssive strength of plain wave fabric composites. Compos Sci Technol 63(5):609-625

61. Wang W, Shenoi RA (2004) Analytical solutions to predict flexural behaviour of curved sandwich beams. J Sandw Struct Mater 6:199-216

62. Hetényi M (1971) Beams on elastic foundation. Theory with applications in the field of civil and mechanical engineering. The University of Michigan Press, Ann Arbor

63. Oller S (2001) Fractura mecánica. Un enfoque global. CIMNE (Centro Internacional de Métodos Numéricos en Ingeniería), Barcelona

64. Timoshenko SP (1940) Strength of materials. Part I. Elementary theory and problems. Van Nostrand, New York

65. Martínez X, Oller S, Barbat A, Rastellini F (2007) New procedure to calculate compression strength of FRP using the serial/parallel mixing theory. In: FRPRCS-8 -8th international symposium on fiber reinforced polymer reinforcement for concrete structures

66. Timoshenko SP (1940) Strength of materials. Part II. Advanced theory and problems. Van Nostrand, New York

67. Martínez X (2008) Micro-mechanical simulation of composite materials using the serial/parallel mixing theory. Ph.D. thesis, Departament de Resistència de Materials i Estructures a l'Enginyeria (RMEE)—UPC. Director: Sergio Oller

68. Hoffman JD (2001) Numerical methods for scientists and engineers. Dekker, New York 